\title{
Relations and radicals in abstract lattices and in lattices of subspaces of Banach spaces and of ideals of Banach algebras. Amitsur's theory revisited.
}

\author{
Edward Kissin, Victor Shulman and Yuri Turovskii
}

July 10, 2019

\begin{abstract}
We refine Amitsur's theory of radicals in complete lattices and apply the obtained results to the theory of radicals in the lattices of subspaces of Banach spaces and in the lattices of ideals of Banach and $\mathrm{C}^{*}$-algebras and of Banach Lie algebras.
\end{abstract}

\section{Introduction}

In his research of the radical theory of algebras and rings, Amitsur [A-II, A-III] discovered that a significant part of the results of this theory can be formulated and proved in terms of the general theory of lattices. In [A-I] he developed the theory of radicals for relations in lattices that was used in various areas of algebra: group theory, non-associative rings, Lie algebras, universal algebras, etc.

Later Dixon [Di1] initiated the radical approach to some problems of functional analysis and laid the basis of the theory of topological radicals of Banach algebras. This theory was further developed and applied to the theory of invariant subspaces of operator algebras and to classification of Banach and operator Lie algebras in [ST], [KST3], [KST5].

In this paper we investigate both aspects of the theory of radicals. In part one: Sections 2 to 7 we revise and refine Amitsur's theory of radicals in complete abstract lattices. In part two: Sections 8 and 9 we apply the obtained results to the theory of radicals in the lattices of subspaces of Banach spaces and in the lattices of ideals of Banach and $\mathrm{C}^{*}$-algebras and of Banach Lie algebras.

Recall that a partially ordered set $(Q, \leq)$ with a reflexive, anti-symmetric, transitive relation $\leq$ is a lattice if all $a, b \in Q$ have a least upper bound $a \vee b$ and a greatest lower bound $a \wedge b$. It is complete if each $G \subseteq Q$ has a least upper bound $\vee G$ and a greatest lower bound $\wedge G$. Set $\mathbf{0}=\wedge Q$ and $\mathbf{1}=\vee Q$. A transitive relation in $Q$ is an order; a relation $\prec_{1}$ in $Q$ is stronger than a relation $\prec$ if

$$
a \prec_{1} b \text { implies } a \prec b \text { for } a, b \in Q \text { (we write } \prec_{1} \subseteq \prec \text { ). }
$$

We will only consider reflexive relations $\ll$ in $(Q, \leq)$ stronger than $\leq$ and denote by $\operatorname{Ref}(Q)$ the family of all such relations. For $x \leq y$ in $Q$, set $[x, y]=\{z \in Q: x \leq z \leq y\}$.

Amitsur [A-I] studied $\mathbf{H}$ - and dual $\mathbf{H}$-relations $\ll$ in complete lattices $Q$ and, using a special procedure, constructed the $\mathbf{R}$-order $\ll^{\triangleright}$ from an $\mathbf{H}$-relation and the dual $\mathbf{R}$-order $\ll^{\triangleleft}$ from a dual H-relation (Definition 3.2). He proved that $\ll^{\triangleright}$ has a unique $\ll^{\triangleright}$-radical $\mathfrak{r}_{[a, b]}$ in each interval $[a, b]$ in $Q$ and $\ll^{\triangleleft}$ has a unique dual $\ll^{\triangleleft}$-radical $\mathfrak{p}_{[a, b]}$. 
In our paper (Theorems 2.4 and 2.5) we establish that even weaker relations ( $\mathbf{T}$ - and dual T-orders) have unique radicals in each $[a, b]$. Moreover, for a $\mathbf{T}$-order, the map $\mathfrak{r}: a \in Q \rightarrow \mathfrak{r}_{[a, \mathbf{1}]}$ is pre-radical map; for a dual T-order, the map $\mathfrak{p}: a \in Q \rightarrow \mathfrak{p}_{[0, a]}$ is a dual pre-radical maps ((2.11) and (2.12)) on $Q$. For $\mathbf{R}$-orders and dual $\mathbf{R}$-orders they are, respectively, radical and dual radical maps $((2.13)$ and $(2.14))$ on $Q$ (Theorems 3.5 and 3.6). If a relation $\ll$ is both a $\mathbf{T}$ - and a dual T-order, it coincides "locally" with $\leq$, i.e., $Q$ is a union of mutually disjoint intervals $\left[a_{\lambda}, b_{\lambda}\right], \lambda \in \Lambda$, such that $x \ll y$ if and only if $x, y \in\left[a_{\lambda}, b_{\lambda}\right]$ for some $\lambda \in \Lambda$, and $x \leq y$ (Theorem 2.12).

In Section 4 we investigate the relations $\ll^{\triangleright}$ and $\ll^{\triangleleft}$ constructed from arbitrary relations $\ll$. We compare them to other naturally constructed relations $\ll^{\text {lo }}$ and $\ll^{\text {up }}$ (Definition 4.2 ) and prove that each interval $[a, b]$ in $Q$ has $\ll^{\triangleright}$ - and dual $\ll^{\triangleleft}$-radicals which are not, however, unique.

For an $\mathbf{H}$-relation $\ll$, we show in Section 5 that $\ll^{\triangleright}$ is an $\mathbf{R}$-order, it coincides with $\ll^{\text {up }}$ and each $[a, b]$ has a unique $\ll^{\triangleright}$-radical. Similarly, for a dual $\mathbf{H}$-relation $\ll, \ll^{\triangleleft}$ is a dual $\mathbf{R}$-order coinciding with $\ll^{\text {lo }}$ and each $[a, b]$ has a unique dual $\ll^{\triangleleft}$-radical. We prove that $\ll$ is an $\mathbf{R}$-order (a dual R-order) if and only if $\ll=\ll^{\triangleright}\left(\ll=\ll^{\triangleleft}\right)$.

In Section 6 we investigate two incompatible relations: the gap $\left(<_{\mathfrak{g}}\right)$ and continuous $\left(<_{\mathfrak{c}}\right)$ relations in lattices $Q$. We show that if $Q$ is modular then $<_{\mathfrak{g}}$ is an $\mathbf{H}$ - and a dual $\mathbf{H}$-relation. If $Q$ is modular and has (JID) and (MID) (see (6.5)) then $<_{\mathfrak{c}}$ is an $\mathbf{R}$ - and a dual $\mathbf{R}$-order and $Q$ is a union of disjoint sets without gaps.

Section 7 is devoted to the study of enveloping and inscribing sets in lattices $Q$. We prove that there is a bijection between all enveloping sets and all radical maps in $Q$, and between all inscribing sets and all dual radical maps in $Q$. We consider some enveloping sets in the lattice $\operatorname{Ref}(Q)$. In particular, we show that the sets of all $\mathbf{T}$-orders, of all dual $\mathbf{T}$-orders, of all $\mathbf{R}$-orders, of all dual $\mathbf{R}$-orders are enveloping in $\operatorname{Ref}(Q)$. We also study transfinite extensions of relations and, in particular, of the relations $<_{\mathfrak{g}}$ and $<_{\mathfrak{c}}$.

An important place in the theory of operator algebras is occupied by the study of lattices of subspaces of Banach spaces $X$ and, especially, of the lattices Lat $\mathcal{A}$ of invariant subspaces of operator algebras $\mathcal{A}$ on $X$. In Section 8 the above results are used to study such lattices.

Denote by $\operatorname{Ln}(X)$ the lattice of all linear subspaces and by $\mathrm{Cl}(X)$ the lattice of all closed subspaces of $X$. In Theorem 8.1 we describe ascending and descending «-series of subspaces in sublattices of $\operatorname{Ln}(X)$ and $\mathrm{Cl}(X)$ with respect to $\mathbf{H}$ - and dual $\mathbf{H}$-relations $\ll$, and their $\ll^{\triangleright}$ - and $\ll^{\triangleleft}$-radicals $\mathfrak{r}$ and $\mathfrak{p}$, respectively. In the sublattices Lat $\mathcal{A}$ of $\mathrm{Cl}(X)$ these radicals are superinvariant (Proposition 8.13), i.e., they are invariant for the operator Lie algebra

$$
\text { Nor } \mathcal{A}=\{S \in B(X): S A-A S \in \mathcal{A} \text { for } A \in \mathcal{A}\} .
$$

We concentrate on the study of the gap relation $<_{\mathfrak{g}}$ and the relations $\left\{\ll_{n}\right\}_{1 \leq n \leq \infty}$ defined in $\operatorname{Ln}(X)$ and $\operatorname{Cl}(X)$ by the condition $L \ll_{n} M$ if $\operatorname{dim}(M / L)<n$. As $\operatorname{Ln}(X)$ is a modular lattice, $<_{\mathfrak{g}}$ and all $\ll_{n}$ are both $\mathbf{H}$ - and dual $\mathbf{H}$-relations (HH-relations) in it (Proposition 8.3 and Corollary 8.5).

The lattice $\mathrm{Cl}(X)$ is not modular and, although all $\ll_{n}$ are still $\mathbf{H H}$-relations in it, there are sublattices where $<_{\mathfrak{g}}$ is neither $\mathbf{H}-$, nor dual $\mathbf{H}$-relation (Corollary 8.21). The main obstacle is the fact that the sum of subspaces from $\mathrm{Cl}(X)$ is not necessarily closed. We construct in $\mathrm{Cl}(X)$ an H-relation $\sqsubset_{\mathfrak{g}}$ and a dual $\mathbf{H}$-relation $\prec_{\mathfrak{g}}$ stronger than $<_{\mathfrak{g}}$ that give us the $\sqsubset_{\mathfrak{g}}^{\triangleright}$-radical and the dual $\prec_{\mathfrak{g}}^{\triangleleft}$-radical in each sublattice of $\mathrm{Cl}(X)$.

For a Hilbert space $X$, we define in (8.27) another class of the HH-relations $\ll_{n}^{\perp}, 0 \leq n \leq \infty$, in $\mathrm{Cl}(X)$ (Theorem 8.26). Moreover, each HH-relation in $\mathrm{Cl}(X)$ is either $\ll_{n}$, or $\ll_{n}^{\perp}$ for some $n$ (see [K3] and Theorem 8.27). Each commutative subspace lattice (CSL) $Q$ in $\mathrm{Cl}(X)$ has properties 
(JID) and (MID) (see (7.15)). So it is modular, $<_{\mathfrak{g}}=\sqsubset_{\mathfrak{g}}=\prec_{\mathfrak{g}}$ is an HH-relation in $Q$, and $Q$ is a union of disjoint intervals each of which has no gaps (Theorem 8.23).

The study of ideals of Banach and Banach Lie algebras constitutes one of the main tools of research. In [KST3] the authors used the structure of chains of ideals in Banach Lie algebras to develop the radical theory of these algebras. In this paper we study chains of ideals generated by $\mathbf{H}$ - and dual $\mathbf{H}$-relations in the lattices $\mathrm{Id}_{A}$ of all closed ideals of Banach algebras $A$.

As $\ll_{\infty}$ is $\mathbf{H H}$-order in $\operatorname{Id}_{A}$, each sublattice of $\operatorname{Id}_{A}$ has the unique $\ll_{\infty}^{\triangleright}$-radical and the dual $\ll_{\infty}^{\triangleleft}$-radical. Denoting by $\Sigma_{A}$ the set of all subalgebras of finite codimension in a Banach algebra $A$, we show in Proposition 9.6 that $\cap_{S \in \Sigma_{A}} S=\cap\left\{I \in \operatorname{Id}_{A}\right.$ : $\left.\operatorname{dim}(A / I)<\infty\right\}$.

The relation $<_{\mathfrak{g}}$ in $\operatorname{Id}_{A}$ is not always an $\mathbf{H}$-, or a dual $\mathbf{H}$-relation, since the sum of closed ideals of $A$ is not necessarily closed. If, however, each ideal of $A$ has a bounded left or right approximate identity, then $I+J$ is closed for all $I, J \in \mathrm{Id}_{A}$, so that $<_{\mathfrak{g}}$ is an HH-relation in $\operatorname{Id}_{A}$ (Corollary 9.2).

Let $L R(A)$ be the operator algebra generated by all operators of left and right multiplication by elements from $A$. Then $\operatorname{Id}_{A}=$ Lat $L R(A)$ is a sublattice of $\operatorname{Cl}(A)$, the Lie algebra Nor $L R(A)$ contains all derivations of $A$ and operators from Nor $L R(A)$ map $\overline{I^{2}}$ in $I, I \in \operatorname{Id}_{A}$ (Theorem 9.5).

If $\cap_{S \in \Sigma_{A}} S=\{0\}$, for a Banach Lie algebra $A$, then (Theorem 9.16) the dual $\ll_{\infty}^{\triangleleft}$-radical $\mathfrak{p}$ in the lattice of all characteristic Lie ideals of $A$ (invariant for all derivations of $A$ ) is $\{0\}$. So there is a descending series $\left(I_{\lambda}\right)_{1 \leq \lambda \leq \gamma}$ of characteristic Lie ideals of $A$ such that $\operatorname{dim}\left(I_{\lambda} / I_{\lambda+1}\right)<\infty$ for $\lambda \neq \gamma, I_{1}=A$ and $\mathfrak{p}=I_{\gamma}=\{0\}$.

Section 9.2 is devoted to the study of $\mathbf{H}$ - and dual $\mathbf{H}$-relations in the lattices $\operatorname{Id}_{A}$ of all ideals of $\mathrm{C}^{*}$-algebras $A$. In this case $\operatorname{Id}_{A}$ is a modular lattice and $<_{\mathfrak{g}}$ is an HH-relation in $\operatorname{Id}_{A}$. $\operatorname{The}$ lattice $\operatorname{Id}_{A}$ has many $\mathbf{H}$-relations that can be obtained in the following way. Let $\mathfrak{A}$ be the set of all $\mathrm{C}^{*}$-algebras. A subclass $P$ of $\mathfrak{A}$ is a property, if $\{0\} \in P$ and $A \in P$ implies $B \in P$ for all $B \approx A$. Each property $P$ generates the relation $\ll_{P}$ in $\operatorname{Id}_{A}$ by $I \ll_{P} J$ if $I \subseteq J$ in $\operatorname{Id}_{A}$ and $J / I \in P$.

A property $P$ is lower stable if $A \in P$ implies $\operatorname{Id}_{A} \subset P ; P$ is upper stable if $A \in P$ implies that the quotients $A / I \in P$ for all $I \in \operatorname{Id}_{A}$. In Theorem 9.7 we prove that $P$ is upper (lower) stable if and only if $\ll_{P}$ is an $\mathbf{H}$-relation (a dual $\mathbf{H}$-relation). Some characteristics of the $\ll_{P}^{\triangleright}$-radicals $\mathfrak{r}_{P}$ and the dual $\ll_{P}^{\triangleleft}$-radicals $\mathfrak{p}_{P}$ in $\operatorname{Id}_{A}$ are discussed in Theorem 9.8. These radicals are invariant for all automorphisms of $A$ (Corollary 9.9). Since many properties in $\mathfrak{A}$ are upper, or lower stable, we have a large variety of $\mathbf{H}$ - and dual $\mathbf{H}$-relations in $\operatorname{Id}_{A}$. These relations were investigated in [KST4]; in this paper we briefly consider some of them.

For example, the classes $C C R$ and $G C R$ of all CCR- and GCR-algebras are lower and upper stable properties, while the class of all NGCR-algebras is a lower, but not upper stable property (see $[\mathrm{D}]$ ). So $\ll_{C C R}$ and $\ll_{G C R}$ are $\mathbf{H H}$-relations. This gives a well-known result that the radical $\mathfrak{r}_{C C R}$ of a $\mathrm{C}^{*}$-algebra $A$ is the largest GCR-ideal and $A / \mathfrak{r}_{C C R}$ has no CCR-ideals. Moreover, if $\mathfrak{r}_{C C R} \nsubseteq I \neq A$ then $J / I$ is a CCR-algebra for some $I \varsubsetneqq J \in \operatorname{Id}_{A}$.

We also consider the classes $R Z$ of all real rank zero, $A F$ of all approximately finite-dimensional and $N U$ of all nuclear $\mathrm{C}^{*}$-algebras. They are lower and upper stable properties, so that $\ll_{R Z}$, $\ll_{A F}$, $\ll_{N U}$ are $\mathbf{H H}$-relations in $\mathrm{Id}_{A}$ for all $\mathrm{C}^{*}$-algebras $A$ (Corollary 9.12). While the relations $\ll_{C C R}$ and $\ll_{R Z}$ are not transitive, the relations $\ll_{A F}$ and $\ll_{N U}$ are transitive. Moreover, they are $\mathbf{R}$-orders in all $\operatorname{Id}_{A}$. So the corresponding radicals are, respectively, the largest $A F$-algebra and the largest nuclear algebra in $A$ (Corollary 9.14) (this result was initially obtained in $[\mathrm{ST}]$ ). 


\section{Radicals and T-orders in complete lattices}

Let $(Q, \leq)$ be a complete lattice. Set $\mathbf{0}=\wedge Q$ and $\mathbf{1}=\vee Q$. For each subset $G \subseteq Q$, its $\wedge$-completion $G^{\wedge}$ and $\vee$-completion $G^{\vee}$ are defined by

$$
G^{\wedge}=\{\wedge N: \varnothing \neq N \subseteq G\} \text { and } G^{\vee}=\{\vee N: \varnothing \neq N \subseteq G\} .
$$

A set $G$ is $\wedge$-complete if $G=G^{\wedge}, \vee$-complete if $G=G^{\vee}$, and complete if $G^{\vee}=G^{\wedge}=G$.

Let $(Q, \leq)$ be a complete lattice, let $x<y$ in $Q$ and let $\ll$ be a relation $\operatorname{in} \operatorname{Ref}(Q)$. Set

$$
\begin{aligned}
(x, y] & =[x, y] \backslash\{x\},[x, y)=[x, y] \backslash\{y\}, \\
{[a, \ll] } & =\{x \in Q: a \ll x\}, \quad[\ll, a]=\{x \in Q: x \ll a\} \text { for each } a \in Q .
\end{aligned}
$$

Definition 2.1 A relation $\ll$ from $\operatorname{Ref}(Q)$ is called up-contiguous if $a \ll b$ implies $[a, b] \subseteq[\ll, b]$;

down-contiguous if $a \ll b$ implies $[a, b] \subseteq[a, \ll]$;

up-expanded if $[a, \ll]$ is $\vee$-complete, down-expanded if $[\ll, a]$ is $\wedge$-complete for all $a \in Q$;

contiguous, expanded if $\ll$ satisfies the corresponding up-and down-condition.

Note that if a relation has one of the properties defined above, its restriction to any interval also has it. We consider now some examples of these relations.

Example 2.2 1) Let $X=[0,1] \subset \mathbb{R}$ and $Q=P(X)$ be its power set - the lattice of all subsets of $X$ with $\leq=\subseteq$. For $A, B \in Q$, we write $A \ll B$ if $A \subseteq B \subseteq \bar{A}$. Then $\ll$ is contiguous: if $A \ll B$ then $[A, B] \subseteq[A, \ll] \cap[\ll, B]$, since $\bar{C}=\bar{A}$ for each $C \in[A, B]$. It is also up-expanded, as $\vee[A, \ll]=\bar{A} \in[A, \ll]$. However, $\ll$ is not down-expanded, as $\wedge[\ll, X]=\varnothing \notin[\ll, X]$.

2) Let $X$ be the set of all Lebesgue measurable subsets of $\mathbb{R}$ and $Q$ be the set of all equivalence classes in $X$. For $A, B \in Q$, we write $A \leq B$ if $\mu(A \backslash B)=0$, and we write $A \ll B$ if $A \leq B$ and $\mu(B \backslash A)<\infty$. Then $\ll$ is contiguous, but neither up-, nor down-expanded.

3) Let $Q_{N}=\{1, \ldots, N\}$ for $N \in N$, the order $\leq$ is defined as usual and $n \ll m$ if $n$ is a divisor of $m$. Then $\ll$ is expanded, but not contiguous.

Definition 2.3 (i) A transitive relation is a $\mathbf{T}$-order if it is up-contiguous and up-expanded;

(ii) A transitive relation is a dual $\mathbf{T}$-order if it is down-contiguous and down-expanded.

(iii) « is a TT-order, if it is a T-and a dual T-order, i.e., « is contiguous and expanded.

By the Duality Principle [Sk, Theorem 1.3'], the results for down-conditions follow from the corresponding results for up-conditions and vice versa. The results for dual T-orders follow from the corresponding results for $\mathbf{T}$-orders and vice versa.

Following $[\mathrm{A}-\mathrm{I}]$, for $\ll \in \operatorname{Ref}(Q)$, define its lower and upper complement relations $\rightleftarrows$ and $\overleftarrow{\ll}$

$$
a \overleftarrow{\ll} b \text { if }[a, \ll] \cap[a, b]=\{a\} ; \text { and } a \gtreqless b \text { if }[\ll, b] \cap[a, b]=\{b\} \text { for } a \leq b \text { in } Q
$$

An element $\mathfrak{r} \in Q$ is called a $\ll$-radical if $\mathbf{0} \ll \mathfrak{r} \overleftarrow{\ll} \mathbf{1}$; an element $\mathfrak{p}$ is called a dual «-radical if $\mathbf{0}$ $\gtrless \mathfrak{p} \ll 1$. In particular, for an interval $[a, b] \subseteq Q$,

$$
\begin{aligned}
& \mathfrak{r} \in[a, b] \text { is a } \ll \text {-radical in }[a, b] \text { if } a \ll \mathfrak{r} \overleftarrow{\ll} b, \\
& \mathfrak{p} \in[a, b] \text { is a dual } \ll \text {-radical in }[a, b] \text { if } a \ll \mathfrak{p} \ll b \text {. }
\end{aligned}
$$


The set of radicals may be empty or have many elements. For example, let $Q=[0,1] \subseteq \mathbb{R}$ and $\ll$ $\in \operatorname{Ref}(Q)$ be such that $x \ll y$ only if $0 \neq x \leq y \neq 1$. Then the set $[a, 1], a \neq 0$, has no «-radicals and the dual $\ll$-radical $\mathfrak{p}=1$; the set $[0, b], b \neq 1$, has no dual $\ll$-radicals and the $\ll$-radical $\mathfrak{r}=0$.

Theorem 2.4 (i) If $\ll$ is an up-expanded order, $\mathfrak{r}=\vee([a, b] \cap[a, \ll])$ is $a \ll$-radical in $[a, b] \subseteq Q$.

(ii) If $\ll$ is a $\mathbf{T}$-order then $\mathfrak{r}$ in (i) is a unique radical and $[a, \mathfrak{r}]=[a, b] \cap[\ll, \mathfrak{r}]$.

(iii) If each $[a, b] \subseteq Q$ has a unique $\ll$-radical then $\ll i s$ up-expanded.

(iv) An up-contiguous order $\ll$ is a $\mathbf{T}$-order if and only if each $[a, b] \subseteq Q$ has a unique «-radical.

Proof. (i) As $\ll$ is up-expanded, $[a, \ll] \cap[a, b]$ is $\vee$-complete. So it contains $\mathfrak{r}$. Hence $a \ll \mathfrak{r}$. If $\mathfrak{r} \ll y$ for some $y \in(\mathfrak{r}, b]$, then $a \ll y$ by transitivity of $\ll-$ a contradiction, since $\mathfrak{r}$ is the largest element in $[a, \ll] \cap[a, b]$. Thus $\mathfrak{r} \overleftarrow{\ll} b$. So $\mathfrak{r}$ is a $\ll$-radical in $[a, b]$.

(ii) Let $\ll$ be also up-contiguous and $a \ll z \overleftarrow{\ll} b$ for some $z$. Then $z \leq \mathfrak{r}$, since $\mathfrak{r}$ is the largest element in $[a, \ll] \cap[a, b]$. As $a \ll \mathfrak{r}$ and $\ll$ is up-contiguous, $[a, \mathfrak{r}] \subseteq[\ll, \mathfrak{r}]$. So $z \ll \mathfrak{r}$. As $z \overleftarrow{\ll} b$, we have $z=\mathfrak{r}$. Thus $\mathfrak{r}$ is a unique $\ll$-radical.

(iii) Let $a \in Q, G \subseteq[a, \ll]$ and $b=\vee G$. Let $\mathfrak{r}$ be the $\ll$-radical in $[a, b]: a \ll \mathfrak{r} \overleftarrow{\ll} b$. If $x \in G$ then $a \ll x \leq b$. Let $\mathfrak{r}_{x}$ be the «-radical in $[x, b]: x \ll \mathfrak{r}_{x} \overleftarrow{\ll} b$. As $\ll$ is an order, $a \ll \mathfrak{r}_{x} \overleftarrow{\ll} b$. So $\mathfrak{r}_{x}$ is a $\ll$-radical in $[a, b]$. As the «-radical in $[a, b]$ is unique, $\mathfrak{r}=\mathfrak{r}_{x}$. Thus $a \ll x \ll \mathfrak{r} \overleftarrow{\ll} b$ for all $x \in G$. Hence $b=\vee G \leq \mathfrak{r}$. Thus $b=\mathfrak{r}$. So $a \ll b$. Therefore $b \in[a, \ll]$, so that $\ll$ is up-expanded.

Part (iv) follows from (ii) and (iii).

By duality we get the following result.

Theorem 2.5 (i) If $\ll$ is a down-expanded order, $\mathfrak{p}=\wedge([a, b] \cap[\ll, b])$ is a dual «-radical in $[a, b]$.

(ii) If $\ll$ is a dual $\mathbf{T}$-order then $\mathfrak{p}$ in (i) is unique and $[\mathfrak{p}, b]=[a, b] \cap[\mathfrak{p}, \ll]$.

(iii) If each $[a, b] \subseteq Q$ has a unique dual $\ll$-radical then $\ll$ is down-expanded.

(iv) A down-contiguous order $\ll$ is a dual $\mathbf{T}$-order if and only if each $[a, b] \subseteq Q$ has a unique dual «-radical.

As the following examples show, the results of Theorems 2.4 and 2.5 can not be strengthened.

Example 2.6 (i) The existence of a unique «-radical in each $[a, b] \subseteq Q$ only guarantees that $\ll$ is up-expanded, but not that it is up-contiguous. Indeed, let $\ll$ be the reflexive relation in $Q=[0,1] \subseteq \mathbb{R}$ with only one non-trivial pair $0 \ll 1$. Each $[a, b] \subseteq Q$ has a unique «-radical: $\mathfrak{r}_{[a, b]}=a$ if $[a, b] \neq[0,1]$, and $\mathfrak{r}_{[0,1]}=1$. The relation $\ll$ is up-expanded, but not up-contiguous (not a T-order): $0 \ll 1$ but $Q=[0,1] \nsubseteq[\ll, 1]=\{0,1\}$.

(ii) The existence of «-radicals in each $[a, b] \subseteq Q$ does not even guarantee that $\ll$ is upexpanded. Indeed, let $Q=\{\mathbf{0}, a, b, \mathbf{1}\}, \mathbf{0}<a<\mathbf{1}$ and $\mathbf{0}<b<\mathbf{1}$. Let $\ll$ be the reflexive relation in $Q$ such that $\mathbf{0} \ll a$ and $\mathbf{0} \ll b$. Then $[\mathbf{0}, \mathbf{1}]$ has two $\ll$-radicals: $\mathfrak{r}_{1}=a$ and $\mathfrak{r}_{2}=b$. The relation $\ll$ is not up-expanded, since $[\mathbf{0}, \ll]=\{\mathbf{0}, a, b\}$ and $\vee[\mathbf{0}, \ll]=a \vee b=\mathbf{1} \notin[\mathbf{0}, \ll]$.

(iii) If $\ll$ is up-expanded but not up-contiguous then all $[a, b] \subseteq Q$ have radicals but not necessarily unique. Indeed, let $\ll$ be the reflexive relation in $Q=[0,1] \subseteq \mathbb{R}$ such that $0 \ll 1$ and $0 \ll \frac{1}{2}$. It is up-expanded, but not up-contiguous (not a T-order): $0 \ll 1$ but $Q=[0,1] \nsubseteq[\ll, 1]=\{0,1\}$. Each $[a, b] \neq[0,1]$ has a unique «-radical, while $[0,1]$ has two «-radicals: $\frac{1}{2}$ and 1 . 
For a dual $\mathbf{T}$-order $\ll$ in $Q$, denote by $\mathfrak{p}(b)$ the unique dual $\ll$-radical in $[\mathbf{0}, b] \subseteq Q$ :

$$
\mathfrak{p}(b)=\mathfrak{p}_{\ll}(b)=\wedge[\ll, b], \mathbf{0} \ll \mathfrak{p}(b) \ll b \text { and }[\mathbf{0}, b] \cap[\mathfrak{p}, \ll]=[\mathfrak{p}, b] .
$$

For a $\mathbf{T}$-order $\ll$ in $Q$, denote by $\mathfrak{r}(a)$ the unique $\ll$-radical in $[a, \mathbf{1}]$ :

$$
\mathfrak{r}(a)=\mathfrak{r}_{\ll}(a)=\vee[a, \ll], a \ll \mathfrak{r}(a) \overleftarrow{\ll} \mathbf{1} \text { and }[a, \mathbf{1}] \cap[\ll, \mathfrak{r}]=[a, \mathfrak{r}]
$$

Lemma 2.7 (i) Let $\ll$ be a dual $\mathbf{T}$-order in $Q$. Then

$$
\begin{aligned}
\mathfrak{p}(\mathfrak{p}(b)) & =\mathfrak{p}(b) \text { for all } b \in Q ; \quad a \ll b \text { implies } \mathfrak{p}(b)=\mathfrak{p}(a) \ll a \\
\mathfrak{p}(b) & \leq a \leq b \text { implies } \mathfrak{p}(b)=\mathfrak{p}(a) \ll a
\end{aligned}
$$

If $\ll$ is also up-contiguous then $\mathfrak{p}(b) \leq a \leq b$ implies $a \ll b$.

(ii) Let $\ll$ be a $\mathbf{T}$-order in $Q$. Then

$$
\begin{aligned}
\mathfrak{r}(\mathfrak{r}(a)) & =\mathfrak{r}(a) \text { for all } a \in Q ; \quad a \ll b \text { implies } b \ll \mathfrak{r}(b)=\mathfrak{r}(a) ; \\
a & \leq b \leq \mathfrak{r}(a) \text { implies } b \ll \mathfrak{r}(b)=\mathfrak{r}(a) .
\end{aligned}
$$

If $\ll$ is also down-contiguous then $a \leq b \leq \mathfrak{r}(a)$ implies $a \ll b$.

Proof. By $(2.6), \mathfrak{p}(\mathfrak{p}(b)) \ll \mathfrak{p}(b) \ll b$. As $\ll$ is transitive, $\mathfrak{p}(\mathfrak{p}(b)) \ll b$. By $(2.6), \mathfrak{p}(b)$ is the smallest element in $[\ll, b]$. Hence $\mathfrak{p}(b) \leq \mathfrak{p}(\mathfrak{p}(b))$. Thus $\mathfrak{p}(\mathfrak{p}(b))=\mathfrak{p}(b)$.

Let $a \ll b$. As $\mathfrak{p}(b)$ is the smallest element in $[\ll, b]$, we have $\mathfrak{p}(b) \leq a \leq b$. So, by $(2.6)$, $\mathfrak{p}(b) \ll a$. Thus $a \ll b$ implies $\mathfrak{p}(b) \ll a$. Hence $\mathfrak{p}(b) \ll a$ implies $\mathfrak{p}(a) \ll \mathfrak{p}(b)$. So $\mathfrak{p}(a) \ll \mathfrak{p}(b) \ll b$. As $\ll$ is transitive, $\mathfrak{p}(a) \ll b$. By $(2.6), \mathfrak{p}(b) \leq \mathfrak{p}(a)$. Thus $\mathfrak{p}(b)=\mathfrak{p}(a)$. As $\mathfrak{p}(a) \ll a$, (2.8) is proved.

Let $\mathfrak{p}(b) \leq a \leq b$. By $(2.6), \mathfrak{p}(b) \ll a$. By $(2.8), \mathfrak{p}(a)=\mathfrak{p}(\mathfrak{p}(b))=\mathfrak{p}(b)$.

If, in addition, $\ll$ is up-contiguous then $\mathfrak{p}(b) \ll b$ and $a \in[\mathfrak{p}(b), b]$ imply $a \ll b$.

Part (ii) can be proved similarly.

Let $g: Q \rightarrow Q$ be a map on $Q$. We say that $g$ is

a pre-radical map if $x \leq g(x)=g(g(x))$ and $x<y<g(x)$ implies $g(y)=g(x)$;

a dual pre-radical map if $g(g(x))=g(x) \leq x$ and $g(x)<y<x$ implies $g(y)=g(x)$;

a radical map if $x \leq g(x)=g(g(x))$ and $x \leq y$ implies $g(x) \leq g(y)$;

a dual radical map if $g(g(x))=g(x) \leq x$ and $x \leq y$ implies $g(x) \leq g(y)$.

Radical maps were considered in [G, Definition I.3.26], where they are called closure operators.

Remark 2.8 Radical maps are pre-radical, while pre-radical maps are not always radical. Indeed, $x<y<f(x)$ implies $f(x) \leq f(y) \leq f(f(x))=f(x)$. So $f(y)=f(x)$ and $f$ is pre-radical.

On the other hand, let $Q=\{\mathbf{0}, a, b, c, d, \mathbf{1}\}, a<b, a<c<d$. Set $f(\mathbf{0})=\mathbf{0}, f(a)=f(b)=b$, $f(c)=f(d)=d, f(\mathbf{1})=\mathbf{1}$. Then $f$ is a pre-radical map, but not a radical map, as $a<c$ does not imply $f(a)=b \leq f(c)=d$. Similar results hold for dual radical and dual pre-radical maps.

For a map $g: Q \rightarrow Q$, define the following relation $\ll^{g}$ in $Q$ :

$$
x \ll^{g} y \text { if } x \leq y \text { and } g(x)=g(y) \text { for } x, y \in Q,
$$


Theorem 2.9 (i) For a dual $\mathbf{T}$-order $\ll$, the map

$$
\mathfrak{p}_{\ll}: b \in Q \mapsto \mathfrak{p}_{\ll}(b)(\text { see }(2.6)) \text { is a dual pre-radical map }
$$

and $\ll \subseteq \ll^{\mathfrak{p}} \ll$ (see (1.1)). If $\ll$ is a contiguous dual $\mathbf{T}$-order then $\ll=\ll^{\mathfrak{p}}$.

(ii) The map $g \mapsto \ll^{g}$ is a bijection from the set of all dual pre-radical maps onto the set of all contiguous dual $\mathbf{T}$-orders. The map $\ll \mapsto \mathfrak{p}_{\ll}$ is its inverse, i.e., $\mathfrak{p}_{\ll^{g}}=g$.

Proof. (i) From (2.8), (2.9) and (2.12) it follows that $\mathfrak{p}$ is a dual pre-radical map.

Let $a \ll b$. By $(2.8), \mathfrak{p}(a)=\mathfrak{p}(b)$. Thus $a \ll^{\mathfrak{p}} b$ by $(2.15)$. So $\ll \subseteq \ll^{\mathfrak{p}}$.

Let $a \ll^{\mathfrak{p}} b$. Then, by $(2.15), \mathfrak{p}(b)=\mathfrak{p}(a) \leq a \leq b$. If $\ll$ is also up-contiguous then, by Lemma 2.7 (i), $a \ll b$, so that $\ll^{\mathfrak{p}}$ is stronger than $\ll$. Thus $\ll=\ll^{\mathfrak{p}}$.

(ii) If $x \ll^{g} y \ll^{g} z$ then $g(x)=g(y)=g(z)$ by $(2.15)$. So $x \ll^{g} z$. Thus $\ll^{g}$ is an order.

Let $x \ll^{g} y$ and $z \in[x, y]$. Then $g(y)=g(x) \leq x \leq z \leq y$ by $(2.12)$ and $g(z)=g(y)=g(x)$. Thus $x \ll^{g} z \ll^{g} y$. So $[x, y] \subseteq\left[x, \ll^{g}\right]$ and $[x, y] \subseteq\left[\ll^{g}, y\right]$, i.e., $\ll^{g}$ is contiguous.

Fix $y \in Q$. By (2.15) and (2.12), $\left[\ll^{g}, y\right]=\{x \in Q: g(y)=g(x) \leq x \leq y\} \subseteq[g(y), y]$. On the other hand, for each $g(y) \leq x \leq y$, it follows from (2.12) that $x \ll^{g} y$. Thus $[g(y), y] \subseteq\left[\ll^{g}, y\right]$. So $\left[\ll^{g}, y\right]=[g(y), y]$. Hence

$$
\mathfrak{p}(y)=\mathfrak{p}_{\ll^{g}}(y)=\wedge\left[\ll^{g}, y\right]=g(y) \in\left[\ll^{g}, y\right] .
$$

Thus $\left[\ll^{g}, y\right]$ is $\wedge$-complete. Hence $\ll^{g}$ is down-expanded. So $\ll^{g}$ is a dual $\mathbf{T}$-order which is also up-contiguous and $\mathfrak{p}_{\ll g}=g$. Applying (i), we complete the proof.

By duality we have the following result.

Theorem 2.10 (i) For a $\mathbf{T}$-order $\ll$, the map $\mathfrak{r}: a \mapsto \mathfrak{r}_{\ll}(a)$ (see (2.7)) is a pre-radical map, and $\ll$ $\subseteq \ll^{\mathfrak{r}}($ see $(1.1))$. If $\ll$ is a contiguous $\mathbf{T}$-order then $\ll=\ll^{\mathfrak{r}}$.

(ii) The map $g \mapsto \ll^{g}$ is a bijection from the set of all pre-radical maps onto the set of all contiguous $\mathbf{T}$-orders. The map $\ll \mapsto \mathfrak{r}_{\ll}$ is its inverse, i.e., $\mathfrak{r}_{\ll g}=g$.

Each TT-order $\ll$ defines the maps $\mathfrak{p}, \mathfrak{r}$ on $Q$ by (2.6) and (2.7). We will show that $Q$ decomposes into a union of disjoint intervals and the restriction of $\ll$ to each of them coincides with $\leq$. Set

$$
Q_{\mathfrak{r}}=\{x \in Q: x=\mathfrak{r}(x)\} \text { and } Q_{\mathfrak{p}}=\{y \in Q: y=\mathfrak{p}(y)\} .
$$

Proposition 2.11 Let $\ll$ be a TT-order from $\operatorname{Ref}(Q)$. Then

(i) $\mathfrak{r}(a)=\mathfrak{r}(\mathfrak{p}(a))$ and $\mathfrak{p}(a)=\mathfrak{p}(\mathfrak{r}(a))$ for all $a \in Q$.

(ii) The map $\mathfrak{p}$ maps $Q$ onto $Q_{\mathfrak{p}}$ and it maps isomorphically $Q_{\mathfrak{r}}$ onto $Q_{\mathfrak{p}}$. The map $\mathfrak{r}$ maps $Q$ onto $Q_{\mathfrak{r}}$ and it maps isomorphically $Q_{\mathfrak{p}}$ on $Q_{\mathfrak{r}}$ and $\left.\mathfrak{r}\right|_{Q_{\mathfrak{r}}}=\left(\left.\mathfrak{p}\right|_{Q_{\mathfrak{p}}}\right)^{-1}$.

(iii) Let $\mathfrak{p}(c) \leq a \leq b \leq \mathfrak{r}(c)$ for some $c \in Q$. Then $a \ll b, \mathfrak{p}(a)=\mathfrak{p}(b)=\mathfrak{p}(c)$ and $\mathfrak{r}(a)=\mathfrak{r}(b)=\mathfrak{r}(c)$. Thus the relations $\leq$ and $\ll$ coincide on $[\mathfrak{p}(c), \mathfrak{r}(c)]$.

Proof. (i) By (2.6) and (2.7), $\mathfrak{p}(a) \ll a \ll \mathfrak{r}(a)$ for $a \in Q$. So (i) follows from (2.8) and (2.10). (ii) By (2.11) and (2.12), $\mathfrak{p}^{2}=\mathfrak{p}$ and $\mathfrak{r}^{2}=\mathfrak{r}$. Hence $y=\mathfrak{p}(x) \in Q_{\mathfrak{p}}$ for $x \in Q$, as $\mathfrak{p}(y)=$ $\mathfrak{p}^{2}(x)=\mathfrak{p}(x)=y$. Similarly, $\mathfrak{r}(x) \in Q_{\mathfrak{r}}$ for $x \in Q$. For $y \in Q_{\mathfrak{p}}$, we have $\mathfrak{r}(y) \in Q_{\mathfrak{r}}$ and, by (i), 
$\mathfrak{p}(\mathfrak{r}(y))=\mathfrak{p}(y)=y$. Similarly, $\mathfrak{p}(x) \in Q_{\mathfrak{p}}$ and $\mathfrak{r}(\mathfrak{p}(x))=x$ for $x \in Q_{\mathfrak{r}}$. From this (ii) follows immediately.

(iii) By (2.6) and (2.7), $\mathfrak{p}(c) \ll \mathfrak{r}(c)$. As $\ll$ is contiguous, $\mathfrak{p}(c) \ll a \ll b \ll \mathfrak{r}(c)$. So, by (2.8) and $(2.10), \mathfrak{p}(a)=\mathfrak{p}(b)=\mathfrak{p}(c)$ and $\mathfrak{r}(a)=\mathfrak{r}(b)=\mathfrak{r}(c)$. Thus $\leq\left.\right|_{[\mathfrak{p}(c), \mathfrak{r}(c)]}$ is stronger than $\left.\ll\right|_{[\mathfrak{p}(c), \mathfrak{r}(c)]}$. As $\ll \subseteq \leq$ in $Q$, we have $\leq\left.\right|_{[\mathfrak{p}(c), \mathfrak{r}(c)]}=\left.\ll\right|_{[\mathfrak{p}(c), \mathfrak{r}(c)]}$.

Recall that subsets $G_{1}$ and $G_{2}$ of $Q$ are disjoint if $G_{1} \cap G_{2}=\varnothing$.

Theorem 2.12 Let $\ll$ belong to $\operatorname{Ref}(Q)$. The following conditions are equivalent.

(i) « is a TT-order.

(ii) $Q=\cup_{\lambda \in Q_{\mathfrak{r}}}[\mathfrak{p}(\lambda), \lambda]$ and all intervals $[\mathfrak{p}(\lambda), \lambda], \lambda \in Q_{\mathfrak{r}}$, are mutually disjoint. Moreover, $x \ll y$ if and only if $x, y \in[\mathfrak{p}(\lambda), \lambda]$ and $x \leq y$ for some $\lambda \in Q_{\mathfrak{r}}$.

(iii) $Q=\cup_{\lambda \in Q_{\mathfrak{p}}}[\lambda, \mathfrak{r}(\lambda)]$ and all intervals $[\lambda, \mathfrak{r}(\lambda)], \lambda \in Q_{\mathfrak{p}}$, are mutually disjoint. Moreover, $x \ll y$ if and only if $x, y \in[\lambda, \mathfrak{r}(\lambda)]$ and $x \leq y$ for some $\lambda \in Q_{\mathfrak{p}}$.

Proof. (ii) $\Rightarrow$ (i). Let $Q$ satisfy (ii). If $x \ll y$ then $[x, y] \subseteq[\mathfrak{p}(\lambda), \lambda]$ for some $\lambda \in Q_{\mathfrak{r}}$. As $\ll$ on $[\mathfrak{p}(\lambda), \lambda]$ coincides with $\leq$, we have $[x, y] \subseteq[\ll, y]$ and $[x, y] \subseteq[x, \ll]$. Thus $\ll$ is contiguous.

We also have $[x, \ll]=[x, \lambda]$ and $[\ll, y]=[\mathfrak{p}(\lambda), y]$. Hence $[x, \ll]$ is $\vee$-complete and $[\ll, y]$ is $\wedge$-complete. Thus $\ll$ is expanded. Finally, if $x \ll y \ll z$ then $x, y, z \in[\mathfrak{p}(\lambda), \lambda]$ for some $\lambda$ and $x \leq y \leq z$. Hence $x \leq z$, so that $x \ll z$. Thus $\ll$ is transitive, so that $\ll$ is a $\mathbf{T T}$-order.

(i) $\Rightarrow$ (ii). For a TT -order $\ll$, let $z \in[\mathfrak{p}(\lambda), \lambda] \cap[\mathfrak{p}(\nu), \nu]$, where $\lambda \neq \nu$ in $Q_{\mathfrak{r}}$. As $\mathfrak{p}(\lambda) \leq z \leq \lambda$, we have $\mathfrak{p}(z)=\mathfrak{p}(\lambda)$ by (2.12). Similarly, $\mathfrak{p}(z)=\mathfrak{p}(\nu)$, so $\mathfrak{p}(z)=\mathfrak{p}(\lambda)=\mathfrak{p}(\nu)$. By Proposition 2.11, $\mathfrak{p}$ maps isomorphically $Q_{\mathfrak{r}}$ on $Q_{\mathfrak{p}}$. Thus $\lambda=\nu$, a contradiction. So all $[\mathfrak{p}(\lambda), \lambda]$ are disjoint.

Let $x \ll y$. Set $\lambda=\mathfrak{r}(y)$. By (2.10), $y \ll \mathfrak{r}(x)=\mathfrak{r}(y)=\lambda$. By (2.11) and (2.16), $\lambda \in Q_{\mathfrak{r}}$. So $\mathfrak{p}(\lambda)=\mathfrak{p}(\mathfrak{r}(x))=\mathfrak{p}(x) \ll x \ll y \ll \lambda$ by Proposition 2.11 and (2.9). Thus $x, y \in[\mathfrak{p}(\lambda), \lambda]$.

Conversely, let $x \leq y$ in $[\mathfrak{p}(\lambda), \lambda]$. By Proposition $2.11, \ll$ and $\leq$ coincide in $[\mathfrak{p}(\lambda), \lambda]$. So $x \ll y$.

Finally, let $x \in Q$. Set $\lambda=\mathfrak{r}(x)$. By (2.10) and Proposition 2.11, $x \ll \lambda \in Q_{\mathfrak{r}}$. Hence, by Proposition 2.11 and $(2.9), \mathfrak{p}(\lambda)=\mathfrak{p}(x) \ll x \ll \lambda$. So $x \in[\mathfrak{p}(\lambda), \lambda]$. Thus $Q=\cup_{\lambda \in Q_{\mathfrak{r}}}[\mathfrak{p}(\lambda), \lambda]$.

Similarly, one can prove (i) $\Leftrightarrow$ (iii).

It should be noted that if $Q=\cup_{\lambda \in \Lambda}\left[a_{\lambda}, b_{\lambda}\right]$ and all intervals $\left[a_{\lambda}, b_{\lambda}\right], \lambda \in \Lambda$, are mutually disjoint, then the relation in $Q$ defined by $x \ll y$ if and only if $x, y \in\left[a_{\lambda}, b_{\lambda}\right]$ and $x \leq y$ for some $\lambda \in \Lambda$, is a TT-order.

\section{Radicals, H-relations and R-orders in complete lattices}

In this section we consider $\mathbf{H}$-relations, and $\mathbf{R}$-orders. We show that many results obtained for $\mathbf{T}$-orders in the previous section can be strengthened for $\mathbf{R}$-orders.

Lemma 3.1 (i) For a relation $\ll$ in $Q$, the following conditions are equivalent:

$1) \ll$ is up-contiguous and $a \wedge b \ll b$ implies $a \ll a \vee b$;

2) $a \ll b$ and $a \leq c$ imply $c \ll b \vee c$ for $a, b, c \in Q$;

3) $a \ll b$ implies $a \vee x \ll b \vee x$ for every $x \in Q$.

(ii) For a relation $\ll$ in $Q$, the following conditions are equivalent:

$1) \ll i s$ down-contiguous and $a \ll a \vee b$ implies $a \wedge b \ll b$; 
2) $a \ll b$ and $c \leq b$ in $Q$ imply $a \wedge c \ll c$;

3) $a \ll b$ implies $a \wedge x \ll b \wedge x$ for every $x \in Q$.

Proof. (i) 1) $\Rightarrow 2$ ). Let $a \ll b$ and $a \leq c$. Then $b \wedge c \in[a, b]$. As $\ll$ is up-contiguous, $b \wedge c \ll b$, so that $c \ll b \vee c$. 2) $\Longleftrightarrow 3$ ) is proved in [A-I, Page 776].

$3) \Rightarrow 1)$. For $c \in[a, b]$ and $a \ll b$, one has $c=a \vee c \ll b \vee c=b$. Thus $\ll$ is up-contiguous. If $a \wedge b \ll b$ then $a=a \vee(a \wedge b) \ll a \vee b$. Part (ii) is proved similarly.

Definition $3.2([\mathrm{~A}-\mathrm{I}])(\mathrm{i}) \ll$ is an $\mathbf{H}$-relation if it satisfies conditions of Lemma $3.1(i)$.

(ii) « is a dual H-relation if it satisfies equivalent conditions of Lemma 3.1(ii).

(iii) $\ll$ is an $\mathbf{H H}$-relation if it is an $\mathbf{H}$ - and a dual $\mathbf{H}$-relation.

(iv) $\ll$ is an $\mathbf{R}$-order if it is an up-expanded $\mathbf{H}$-order.

(v) «is a dual $\mathbf{R}$-order if it is a down-expanded dual $\mathbf{H}$-order.

(vi) $\ll$ is an $\mathbf{R R}$-order if it is an $\mathbf{R}$-order and a dual $\mathbf{R}$-order.

By the Duality Principle [Sk, Theorem 1.3'], the results for $\mathbf{H}$-relations and $\mathbf{R}$-orders follow from the corresponding results for dual $\mathbf{H}$-relations and dual $\mathbf{R}$-orders and vice versa.

Each $\mathbf{H}$-order $\ll$ is finitely up-expanded: $b, c \in[a, \ll]$ implies $b \vee c \in[a, \ll]$ for all $a \in Q$. Indeed, by Lemma 3.1, $c=a \vee c \ll b \vee c$. By transitivity, $a \ll b \vee c$, as $a \ll c$. Similarly, each dual H-order $\ll$ is finitely down-expanded, i.e., $a, c \in[\ll, b]$ implies $a \wedge c \in[\ll, b]$ for each $a \in Q$.

The notions of (dual) $\mathbf{R}$-orders are stronger than the notions of (dual) $\mathbf{H}$-orders, respectively, as they require up- and down-expandedness and not only its "finite" version. Amitsur [A-I] defined them in a different, but equivalent way (he called them $\mathbf{R}$ - and dual $\mathbf{R}$-relations).

Comparing Definitions 2.3 and 3.2, we see that (dual) $\mathbf{R}$-orders are (dual) $\mathbf{T}$-orders. Thus the results for (dual) $\mathbf{T}$-orders hold also for (dual) $\mathbf{R}$-orders. In particular,

1) for a dual $\mathbf{R}$-order $\ll, \mathfrak{p}(b)=\wedge[\ll, b]$ is the unique dual $\ll$-radical in $[\mathbf{0}, b]$ and $(2.6)$ holds;

2) for an $\mathbf{R}$-order $\ll, \mathfrak{r}(a)=\vee[a, \ll]$ is the unique $\ll$-radical in $[a, \mathbf{1}]$ and $(2.7)$ holds.

However, as the following example shows, (dual) $\mathbf{T}$-orders are not necessarily (dual) $\mathbf{R}$-orders.

Example 3.3 (i) Let $Q=\{\mathbf{0}, a, b, \mathbf{1}\}, a \wedge b=\mathbf{0}$ and $a \vee b=\mathbf{1}$. Let $\ll$ be a reflexive relation in $Q$.

1) If only $\mathbf{0} \ll a$ then $\ll$ is a $\mathbf{T T}$-order but not an $\mathbf{R}$-order as $b=\mathbf{0} \vee b \nless a \vee b=\mathbf{1}$.

2) If only $a \ll \mathbf{1}$ then $\ll$ is a $\mathbf{T T}$-order but not a dual $\mathbf{R}$-order.

(ii) Let $Q=[0,1] \subset \mathbb{R},\left.\ll\right|_{[0,1)}=\leq\left.\right|_{[0,1)}$ and $1 \ll 1$. Then $\ll$ is an $\mathbf{H}$-order but not an $\mathbf{R}$-order.

We consider now some additional properties of the maps $a \mapsto \mathfrak{p}(a), a \mapsto \mathfrak{r}(a)$ (see Lemma 2.7).

Lemma 3.4 (i) Let $\ll$ be a dual $\mathbf{R}$-order. Then (2.8) and (2.9) hold and, for $a, b \in Q$,

$$
\mathfrak{p}(a \wedge b) \ll \mathfrak{p}(a) \wedge \mathfrak{p}(b) \ll a \wedge b \text { and } \mathfrak{p}(a) \leq \mathfrak{p}(b) \text { if } a \leq b
$$

(ii) Let $\ll$ be an $\mathbf{R}$-order. Then (2.10) holds and, for $a, b \in Q$,

$$
a \vee b \ll \mathfrak{r}(a) \vee \mathfrak{r}(b) \ll \mathfrak{r}(a \vee b) \text { and } \mathfrak{r}(a) \leq \mathfrak{r}(b) \text { if } a \leq b
$$


Proof. As a dual $\mathbf{R}$-order is a dual T-order, the results of Lemma 2.7(i) hold for them.

As $\mathfrak{p}(b) \ll b$ and $\mathfrak{p}(a) \ll a$ by (2.6), we have $\mathfrak{p}(a) \wedge \mathfrak{p}(b) \ll \mathfrak{p}(a) \wedge b \ll a \wedge b$ from Lemma 3.1(ii). By transitivity, $\mathfrak{p}(a) \wedge \mathfrak{p}(b) \ll a \wedge b$. So, by $(2.8), \mathfrak{p}(a \wedge b) \ll \mathfrak{p}(a) \wedge \mathfrak{p}(b) \ll a \wedge b$.

If $a \leq b$ then $\mathfrak{p}(a)=\mathfrak{p}(a \wedge b) \leq \mathfrak{p}(a) \wedge \mathfrak{p}(b)$. So $\mathfrak{p}(a) \leq \mathfrak{p}(b)$. The proof of (ii) is similar.

Recall that, for a map $g: Q \rightarrow Q$, the relation $\ll^{g}$ in $Q$ is defined in (2.15).

Theorem 3.5 (i) For a dual $\mathbf{R}$-order $\ll$, the map $\mathfrak{p}_{\ll}$ : $b \mapsto \mathfrak{p}_{\ll}(b)$ in (2.6) is a dual radical map and $\ll \subseteq \ll^{\mathfrak{p} \ll}$ (see (1.1)). If $\ll$ is a contiguous dual $\mathbf{R}$-order then $\ll=\ll^{\mathfrak{p}} \ll$.

(ii) The map $g \mapsto \ll^{g}$ is a bijection from the set of all dual radical maps onto the set of all contiguous dual $\mathbf{R}$-orders. The map $\ll \mapsto \mathfrak{p}_{\ll}$ is its inverse, i.e., $\mathfrak{p}_{\ll g}=g$.

Proof. (i) By (2.8) and (2.6), $\mathfrak{p}(\mathfrak{p}(b))=\mathfrak{p}(b) \leq b$ for $b \in Q$. By Lemma 3.4, $\mathfrak{p}(a) \leq \mathfrak{p}(b)$ if $a \leq b$. Thus $\mathfrak{p}$ satisfies (2.14), so that it is a dual radical map.

If $\ll$ is a dual $\mathbf{R}$-order, it is a dual $\mathbf{T}$-order. So it follows from Theorem 2.9 that $\ll \subseteq \ll^{\mathfrak{p}}$ and that $\ll=\ll^{\mathfrak{p}}$ if $\ll$ is also up-contiguous.

(ii) If $g$ is a dual radical map then it is pre-dual by Remark 2.8 . Hence, by Theorem $2.9, \ll^{g}$ is a contiguous dual $\mathbf{T}$-order and $\mathfrak{p}_{\ll g}=g$.

If $a \ll^{g} b$ then $g(a)=g(b)$. Let $c \leq b$. By (2.14), $g(c) \leq g(b)$ and $g(x) \leq x$ for all $x \in Q$. Thus

$$
g(c)=g(b) \wedge g(c)=g(a) \wedge g(c) \leq a \wedge c \leq c .
$$

So $g(c) \leq a \wedge c \leq c$. As $g$ is also pre-dual, $g(c)=g(a \wedge c)$ by (2.12). Hence $a \wedge c \ll^{g} c$. Thus we have from Lemma 3.1(ii) that $\ll^{g}$ is a dual $\mathbf{H}$-relation. So $\ll^{g}$ is a contiguous dual R-order. Applying (i), we complete the proof.

By duality we get the following result.

Theorem 3.6 (i) For a $\mathbf{R}$-order $\ll$, the map $\mathfrak{r}: a \mapsto \mathfrak{r}(a)$ in $(2.7)$ is a radical map and $\ll \subseteq \ll^{\mathfrak{r}}$ (see(1.1)). If $\ll$ is a contiguous $\mathbf{R}$-order then $\ll=\ll^{\mathfrak{r}}$.

(ii) The map $g \mapsto \ll^{g}$ is a bijection from the set of all radical maps onto the set of all contiguous $\mathbf{R}$-orders. The map $\ll \mapsto \mathfrak{r}_{\ll}$ is its inverse, i.e., $\mathfrak{r}_{\ll g}=g$.

We will now study properties of the maps $\mathfrak{p}, \mathfrak{r}$ for relations satisfying combined conditions.

Lemma 3.7 (i) If $\ll$ is an $\mathbf{H}$-relation and a dual $\mathbf{T}$-order then $\mathfrak{p}(a \vee b) \ll \mathfrak{p}(a) \vee \mathfrak{p}(b)$ for $a, b \in Q$.

(ii) If $\ll i s$ an $\mathbf{H}$-relation and a dual $\mathbf{R}$-order, then $\mathfrak{p}(a \vee b)=\mathfrak{p}(a) \vee \mathfrak{p}(b)$ for $a, b \in Q$.

(iii) If $\ll$ is a dual $\mathbf{H}$-relation and a $\mathbf{T}$-order then $\mathfrak{r}(a) \wedge \mathfrak{r}(b) \ll \mathfrak{r}(a \wedge b)$ for $a, b \in Q$.

(iv) If $\ll$ is a dual $\mathbf{H}$-relation and an $\mathbf{R}$-order then $\mathfrak{r}(a) \wedge \mathfrak{r}(b)=\mathfrak{r}(a \wedge b)$ for $a, b \in Q$.

Proof. (i) By (2.6), $\mathfrak{p}(a) \ll a$. As $\ll$ is an H-relation, $\mathfrak{p}(a) \vee \mathfrak{p}(b) \ll a \vee \mathfrak{p}(b)$ by Lemma 3.1. Similarly, $\mathfrak{p}(b) \ll b$ implies $a \vee \mathfrak{p}(b) \ll a \vee b$. By transitivity of $\ll, \mathfrak{p}(a) \vee \mathfrak{p}(b) \ll a \vee b$. Since $\ll$ is also a dual T-order, $\mathfrak{p}(a \vee b) \ll \mathfrak{p}(a) \vee \mathfrak{p}(b)$ by $(2.8)$.

(ii) If $\ll$ is a dual R-order, $\mathfrak{p}(a) \vee \mathfrak{p}(b) \leq \mathfrak{p}(a \vee b)$ by Lemma 3.4. So the proof follows from (i). Parts (iii) and (iv) follow from duality.

Recall that $\mathbf{T T}$-orders are $\mathbf{T}$ - and dual $\mathbf{T}$-orders, and $\mathbf{R R}$-orders are $\mathbf{R}$ - and dual $\mathbf{R}$-orders. Each TT-order defines both maps $\mathfrak{r}: a \mapsto \mathfrak{r}(a)$ and $\mathfrak{p}: a \mapsto \mathfrak{p}(a)$ on $Q$. In terms of them we will find necessary and sufficient conditions for a $\mathbf{T T}$-order to be an $\mathbf{R}$-, or a dual $\mathbf{R}$-, or an $\mathbf{R R}$-order. 
Corollary 3.8 (i) Let $\ll$ be a $\mathbf{T T}$-order in $Q$. The following conditions are equivalent.

1) $\ll$ is a dual $\mathbf{R}$-order;

2) $\mathfrak{p}(a) \leq \mathfrak{p}(b)$ if $a \leq b$ in $Q$;

3) $\mathfrak{p}(a \wedge b) \leq \mathfrak{p}(a) \wedge \mathfrak{p}(b)$ for $a, b \in Q$;

4) $\mathfrak{p}(a \wedge b)=\mathfrak{p}(\mathfrak{p}(a) \wedge \mathfrak{p}(b))$ for $a, b \in Q$.

(ii) Let $\ll$ be a $\mathbf{T T}$-order in $Q$. The following conditions are equivalent:

$1) \ll$ is an $\mathbf{R}$-order;

2) $\mathfrak{r}(a) \leq \mathfrak{r}(b)$ if $a \leq b$ in $Q$

3) $\mathfrak{r}(a) \vee \mathfrak{r}(b) \leq \mathfrak{r}(a \vee b)$ for $a, b \in Q$

4) $\mathfrak{r}(\mathfrak{r}(a) \vee \mathfrak{r}(b))=\mathfrak{r}(a \vee b)$ for $a, b \in Q$.

(iii) $A$ TT-order $\ll$ is an $\mathbf{R R}$-order if and only if $\mathfrak{p}(a) \leq \mathfrak{p}(b)$ and $\mathfrak{r}(a) \leq \mathfrak{r}(b)$ for all $a \leq b$.

Proof. (i) 1) $\Rightarrow$ 2) follows from Lemma 3.4(i).

2) $\Rightarrow 3)$ As $a \wedge b \leq a$, we have $\mathfrak{p}(a \wedge b) \leq \mathfrak{p}(a)$ from 2). Similarly, $\mathfrak{p}(a \wedge b) \leq \mathfrak{p}(b)$ and 3) follows.

$3) \Rightarrow 4)$ We have $\mathfrak{p}(a \wedge b) \leq \mathfrak{p}(a) \wedge \mathfrak{p}(b) \leq a \wedge b$. By (2.9), $\mathfrak{p}(a \wedge b)=\mathfrak{p}(\mathfrak{p}(a) \wedge \mathfrak{p}(b))$.

$4) \Rightarrow 3)$. As $\mathfrak{p}(x) \leq x$ for $x \in Q$, we have $\mathfrak{p}(\mathfrak{p}(a) \wedge \mathfrak{p}(b)) \leq \mathfrak{p}(a) \wedge \mathfrak{p}(b)$.

$3) \Rightarrow 1$ ). Let $x \ll y$ and $z \in Q$. By Theorem 2.12, $\mathfrak{p}(\lambda) \leq x \leq y \leq \lambda$ and $\mathfrak{p}(\mu) \leq z \leq \mu$ for some $\lambda, \mu \in Q_{\mathfrak{r}}$. Hence, by 3$)$,

$$
\mathfrak{p}(\lambda \wedge \mu) \leq \mathfrak{p}(\lambda) \wedge \mathfrak{p}(\mu) \leq x \wedge z \leq y \wedge z \leq \lambda \wedge \mu \leq \mathfrak{r}(\lambda \wedge \mu)
$$

By Proposition 2.11(iii), $x \wedge z \ll y \wedge z$. Thus $\ll$ is a dual $\mathbf{H}$-relation. So it is a dual $\mathbf{R}$-order.

Part (ii) can be proved similarly. Part (iii) follows from (i) and (ii).

Maps $f, g$ on $Q$ are conjugate if $g(x)=g(f(x))$ and $f(x)=f(g(x))$ for all $x \in Q$.

Corollary 3.9 (i) For an $\mathbf{R R}$-order $\ll$, the maps $\mathfrak{r}_{\ll}$ and $\mathfrak{p}_{\ll}$ are conjugate.

(ii) Let maps $f, g$ on $Q$ be conjugate and let

$$
g(x) \leq x \leq f(x) \text { for } x \in Q \text {, and } f(x) \leq f(y) \text { and } g(x) \leq g(y) \text { for } x \leq y \text { in } Q .
$$

Then there is an $\mathbf{R R}$-order $\ll$ such that $f=\mathfrak{r}_{\ll}$ and $g=\mathfrak{p}_{\ll}$. Moreover, $\ll=\ll^{f}=\ll^{g}$.

Proof. (i) Let $\ll$ be an $\mathbf{R R}$-order. By Theorems 3.5 and $3.6, \mathfrak{r}_{\ll}$ is a radical map and $\mathfrak{p}_{\ll}$ is a dual radical map. By $(2.6)$ and $(2.7), \mathfrak{p}_{\ll}(x) \ll x \ll \mathfrak{r}_{\ll}(x)$ for all $x \in Q$, and

$$
\mathfrak{p}_{\ll}(x) \stackrel{(2.8)}{=} \mathfrak{p}_{\ll}\left(\mathfrak{r}_{\ll}(x)\right) \text { and } \mathfrak{r}_{\ll}(x) \stackrel{(2.10)}{=} \mathfrak{r}_{\ll}\left(\mathfrak{p}_{\ll}(x)\right)
$$

(ii) As $f$ and $g$ are conjugate, $f(f(x))=f(g(f(x)))=f(g(x))=f(x)$. Similarly, $g(g(x))=g(x)$. From this and from (3.1) we get that $f$ is a radical and $g$ is a dual radical map ((2.13), (2.14)).

Let $x \ll^{f} y$. By (2.15), $f(x)=f(y)$. As $f$ and $g$ are conjugate, $g(x)=g(f(x))=g(f(y))=$ $g(y)$. So $x \ll^{g} y$. Similarly, $x \ll^{g} y \Rightarrow x \ll^{f} y$. Thus $\ll^{f}=\ll^{g}$. By Theorems 3.5 and $3.6, \ll=$ $\ll^{f}=\ll^{g}$ is an RR-order, $f=\mathfrak{r}_{\ll}$ and $g=\mathfrak{p}_{\ll}$. 


\section{Construction of contiguous and expanded relations}

We proved in the previous section that $\mathbf{T}$-orders have unique radicals. However, majority of relations in applications are neither contiguous, nor expanded. In this section we consider two ways to construct from a relation some new relations that are contiguous, or expanded, or both, so that

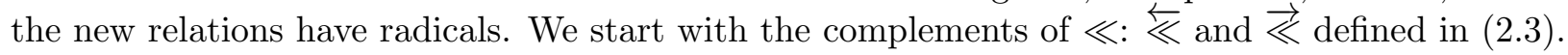

Proposition 4.1 (i) $\overleftarrow{\ll}$ is a down-contiguous relation; the set $[a, \overleftarrow{\ll}]$ is $\wedge$-complete for each $a \in Q$

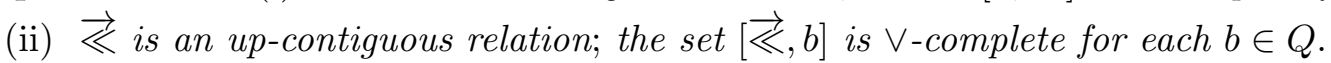

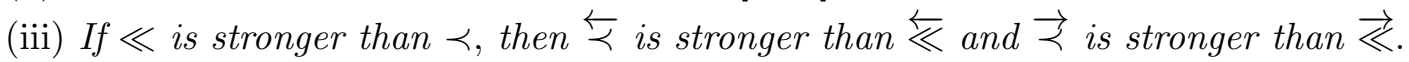

Proof. (i) Let $a \overleftarrow{\ll} b$. By $(2.3),[a, \ll] \cap[a, b]=\{a\}$. Then $[a, \ll] \cap[a, x]=\{a\}$ for each $x \in[a, b]$.

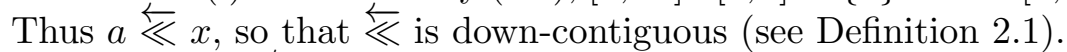

Let $G \subseteq[a, \overleftarrow{\ll}]$. By $(2.3),[a, \ll] \cap[a, b]=\{a\}$ for all $b \in G$. Thus $[a, \ll] \cap[a, \wedge G]=\{a\}$. Hence $a \overleftarrow{\ll} \wedge G$, so that $[a, \overleftarrow{\ll}]$ is $\wedge$-complete. Part (ii) is proved similarly.

(iii) Let $a \overleftarrow{\prec} b$. By $(2.3),[a, \prec] \cap[a, b]=\{a\}$. As $\ll \subseteq \prec$ (see $(1.1)$ ), we have $[a, \ll] \subseteq[a, \prec]$. Hence $[a, \ll] \cap[a, b]=\{a\}$. By $(2.3), a \overleftarrow{\ll} b$. The proof of the second statement is similar.

We introduce now two constructions that play important part in this paper.

Definition 4.2 Let $\ll$ be a relation from $\operatorname{Ref}(Q)$. We say that $G \subseteq Q$ is

1) a lower $\ll$-set if, for each $x \in G \backslash\{\wedge G\}$, there is $y \in G$ such that $x \neq y$ and $y \ll x$;

2) an upper $\ll$-set if, for each $x \in G \backslash\{\vee G\}$, there is $y \in G$ such that $x \neq y$ and $x \ll y$.

3) We write $a \ll^{\text {lo }} b$ if $[a, b]$ is a lower $\ll-$ set; and $a \ll^{\text {up }} b$ if $[a, b]$ is an upper $\ll-$ set.

Proposition $4.3(\mathrm{i}) \ll^{\mathrm{lo}}=\overleftarrow{(\overleftrightarrow{\ll})}$ is a down-contiguous order. If $\ll$ is down-contiguous, $\ll \subseteq \ll^{\mathrm{lo}}$.

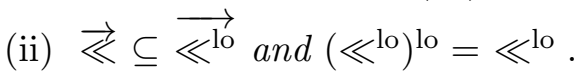

(iii) $\ll^{\mathrm{up}}=\overrightarrow{(\overleftarrow{\ll})}$ is an up-contiguous order. If $\ll$ is up-contiguous, $\ll \subseteq \ll^{\text {up }}$.

(iv) $\overleftarrow{\ll} \subseteq \overleftarrow{\ll \text { up }}$ and $\left(\ll^{\text {up }}\right)^{\text {up }}=\ll^{\text {up }}$

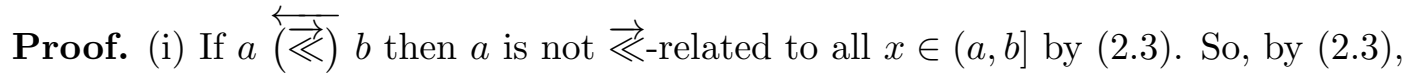

$a \overleftarrow{(\overleftrightarrow{\ll})} b$ if and only if, for each $x \in(a, b]$, there is $y \in[a, x)$ such that $y \ll x$

That is, if and only if $[a, b]$ is a lower $\ll$-set: $a \ll^{\text {lo }} b$. Hence $\ll^{\text {lo }}=\overleftarrow{(\vec{\ll})}$. So, by Proposition 4.1(i), $\ll^{\text {lo }}$ is down-contiguous. Clearly, it is an order.

If $\ll$ is down-contiguous and $a \ll b$ then $[a, b] \subseteq[a, \ll]$. So $a \ll x$ for all $x \in(a, b]$. Thus $[a, b]$ is a lower $\ll$-set: $a \ll^{\text {lo }} b$. Thus $\ll \subseteq \ll^{\text {lo }}$. The proof of (iii) is similar.

(ii) By Proposition 4.1(ii), $\vec{\ll}$ is up-contiguous. So, by (iii) and (i), $\vec{\ll} \subseteq(\vec{\ll})^{\text {up }}=\overrightarrow{(\vec{\ll})}=\vec{\ll}$.

Hence, by (i) and by Proposition 4.1(iii), $\left(\ll^{\mathrm{lo}}\right)^{\mathrm{lo}}=\overleftrightarrow{\ll_{\mathrm{lo}}^{\mathrm{l}}} \subseteq \overleftarrow{(\ll)}=\ll^{\mathrm{lo}}$. 
Let $a$ be not $\overleftrightarrow{\ll^{\mathrm{lo}}}$-related to $b$. By $(4.1)$, there is $x \in(a, b]$ such that $y$ is not $\ll^{\text {lo }}$-related to $x$ for each $y \in[a, x)$. In particular, $a$ is not $\ll^{\mathrm{lo}}$-related to $x$. Then there is $z \in(a, x]$ such that $u \nless z$ for each $u \in[a, z)$. Hence $a$ is not $\ll^{\mathrm{lo}}-$ related to $b$. Thus $\ll^{\mathrm{lo}} \subseteq \overleftrightarrow{\ll^{\mathrm{lo}}}=\left(\ll^{\mathrm{lo}}\right)^{\mathrm{lo}}$. So $\ll^{\mathrm{lo}}=\left(\ll^{\mathrm{lo}}\right)^{\mathrm{lo}}$. The proof of part (iv) is similar.

To describe the second way of constructing new relations from $\ll$, we study chains in lattices. Recall that a chain $C$ in $Q$ is a linearly ordered subset: either $x \leq y$ or $y \leq x$ for $x, y \in C$. A chain $C$ is from $a$ to $b$ if $\wedge C=a$ and $\vee C=b$. A chain $C$ is maximal in $G \subseteq Q$ if $C \subseteq G$ and $G$ has no larger chains.

Definition 4.4 Let $\ll$ be a relation in $Q$ and $C$ be a chain from a to $b$. Then

1) $C$ is a lower (upper) «-chain, if it is a lower (upper) «-set.

2) $C$ is a lower $\ll-g a p$ chain if each $x \in C \backslash\{a\}$ has an immediate $\ll$-predecessor $p_{x} \in C$ : $p_{x} \neq x, p_{x} \ll x$ and $\left[p_{x}, x\right] \cap C=\left\{p_{x}, x\right\}$.

3) $C$ is an upper $\ll$-gap chain if each $x \in C \backslash\{b\}$ has an immediate $\ll$-successor $s_{x} \in C$ : $x \neq s_{x}, x \ll s_{x}$ and $\left[x, s_{x}\right] \cap C=\left\{x, s_{x}\right\}$.

Lemma 4.5 Let $G$ be $a \wedge$-complete set in $Q, C$ be a chain in $G$ and $b:=\vee C \in G$. Set $a=\wedge C$.

(i) There is a maximal chain in $G \cap[a, b]$ containing $C$.

(ii) If $C$ is a maximal chain in $G \cap[a, b]$, it is $\wedge$-complete. If $G$ is complete, $C$ is complete.

(iii) (cf. Lemma I.34 [G]) If $C$ is a $\wedge$-complete lower $\ll-$ gap chain and $b \in C$, then $C$ is complete.

Proof. (i) Apply Zorn's Lemma to the set of all chains in $G \cap[a, b]$ that contain $C$.

(ii) As $C^{\wedge}$ is a chain in $G \cap[a, b]$ containing $C$ and $C$ is maximal, $C=C^{\wedge}$. If $G$ is complete then $C^{\vee} \subseteq G \cap[a, b]$. As $C^{\vee}$ is a chain containing $C$ and $C$ is maximal, $C=C^{\vee}$. So $C$ is complete.

(iii) For $\Gamma \subseteq C, \Gamma \neq\{a\}$, let $K=\{x \in C: y \leq x$ for all $y \in \Gamma\}$. As $b \in K, K \neq \varnothing$. As $C$ is $\wedge$-complete, $d:=\wedge K \in C$. Then $a<d$ and $y \leq d$ for all $y \in \Gamma$. So $d \in K$. As $C$ is a lower «-gap chain, there is an immediate «-predecessor $p$ of $d$ in $C: C \cap[p, d]=\{p, d\}$. If $y \leq p$ for all $y \in \Gamma$, then $p \in K$ and $d=\wedge K \leq p$, a contradiction. Hence there is $y \in \Gamma$ such that $p<y \leq d$. So $y=d$, i.e., $\vee \Gamma=d \in C$. Thus $C$ is $\vee$-complete.

We shall now consider a particular type of extension of lower «-gap chains.

Definition 4.6 Let $D$ be a chain, $a=\wedge D$ and $b=\vee D \in D$. A chain $C$ down-extends $D$ if

$$
C \subseteq[\mathbf{0}, b] \text { and } D=C \cap[a, b] \text {, i.e., } C=D \cup E \text {, where } E \text { is a chain in }[\mathbf{0}, a] .
$$

A chain $D$ is a maximal down-extended lower «-gap chain (resp. lower «-chain), if there does not exist another lower «-gap chain (resp. lower «-chain) that down-extends D.

Let $Q=[0,1] \subset \mathbb{R}$. Then $(Q, \leq)$ is a maximal chain, a maximal down-extended lower $\leq$-chain, but not a lower $\leq$-gap chain. The set $\mathfrak{Q}_{[0,1]}$ of all rational numbers in $[0,1]$ is a maximal downextended lower $\leq$-chain, but is neither a maximal lower $\leq$-chain, nor a lower $\leq$-gap chain. The set $\{0\} \cup \mathfrak{Q}_{\left[\frac{1}{2}, 1\right]}$ is a maximal down-extended lower $\leq$-chain but not a lower $\leq$-gap chain; $\mathfrak{Q}_{\left[\frac{1}{2}, 1\right]}$ is a lower $\leq$-chain but not maximal down-extended. The sets $\{0,1\}$ and $\left\{0, \frac{1}{2}, 1\right\}$ are maximal down-extended lower $\leq$-gap chains and $\left\{\frac{1}{2}, 1\right\}$ is a lower $\ll$-gap chain but not maximal down-extended. 
Proposition 4.7 Let $G$ be a $\wedge$-complete set in $Q$, let $S$ be a complete, lower «-gap chain in $G$ and $b:=\vee G \in S$. Then there is a maximal down-extended complete, lower «-gap chain $C$ in $G$ that

1) down-extends $G$ and $G \cap[\ll, \mathfrak{p}]=\{\mathfrak{p}\}$, where $\mathfrak{p}=\wedge C \in C$.

If $G$ is a lower $\ll-$ set, there is a complete lower $\ll-$ gap chain $C$ in $G$ such that $\wedge G=\wedge C=\mathfrak{p}$ and $\vee C=b$.

Proof. Let $\Gamma_{S}$ be the set of all complete, lower «-gap chains in $G$ that down-extend $S$. It is partially ordered by the inclusion "down-extends". Let $\Omega$ be a linearly ordered subset of $\Gamma_{S}$. Set $K=\cup_{C \in \Omega} C$ and $T=K \cup\{\wedge K\}$. Then $T$ is a chain in $G$ and it down-extends each $D \in \Omega$, as

$$
T \cap[\wedge D, b]=\left(\cup_{C \in \Omega} C\right) \cap[\wedge D, b]=\cup_{C \in \Omega}(C \cap[\wedge D, b]) \stackrel{(4.2)}{=} D .
$$

Let $x \in K$ and $\wedge K<x$. Then $x \in D$ and $\wedge D<x$ for some $D \in \Omega$. As $D$ is a lower «-gap chain, there is $p \in D$ such that $p \ll x \neq p$ and $D \cap[p, x]=\{p, x\}$. By the above,

$$
T \cap[p, x]=T \cap([\wedge D, b] \cap[p, x])=D \cap[p, x]=\{p, x\},
$$

so that $T$ is a lower «-gap chain in $G$ that down-extends $S$.

To prove that $T$ is $\wedge$-complete, let $E \subseteq T$. If $\wedge K \in E$ then $\wedge E=\wedge K \in T$. If $\wedge K \notin E$, set $E_{C}=C \cap E$ for $C \in \Omega$. As each $C$ is complete, $e_{C}:=\wedge E_{C} \in C$. If all $e_{C}$ lie in a chain $D \in \Omega$ then, as $D$ is complete,

$$
\wedge E \stackrel{(5.4)}{=} \wedge_{C \in \Omega}\left(\wedge E_{C}\right)=\wedge_{C \in \Omega} e_{C} \in D \subseteq T .
$$

If not then, for each $D \in \Omega$, there is $C \in \Omega$ such that $e_{C} \notin D$. Thus $\wedge C \leq e_{C}<\wedge D$. Hence

$$
\wedge K \stackrel{(5.4)}{=} \wedge_{C \in \Omega}(\wedge C) \leq \wedge_{C \in \Omega} e_{C} \leq \wedge_{D \in \Omega} \wedge D=\wedge K
$$

Therefore $\wedge E=\wedge_{C \in \Omega} e_{C}=\wedge K \in T$, so that $T$ is $\wedge$-complete. By Lemma 4.5(iii), $T$ is complete. Thus $T \in \Gamma_{S}$ and $T$ is a supremum of $\Omega$. By Zorn's lemma, $\Gamma_{S}$ has a maximal element - a complete, lower $\ll$-gap chain $C$ in $G$ that down-extends $S$.

Set $\mathfrak{p}=\wedge C$. As $C$ is complete, $\mathfrak{p} \in C \subseteq G$. If $x \ll \mathfrak{p}$ for some $x \in G, \mathfrak{p} \neq x$, then $C \cup\{x\} \in \Gamma_{S}$ and larger than $C$. As $C$ is maximal, such $x$ does not exist. So $G \cap[\ll, \mathfrak{p}]=\{\mathfrak{p}\}$.

Let $G$ be a lower $\ll$-set. If $\mathfrak{p} \neq \wedge G$ then there is $x \in G$ such that $x \neq \mathfrak{p}$ and $x \ll \mathfrak{p}$ which contradicts (4.3). Hence $\mathfrak{p}=\wedge G$. The chain $S=\{b\}$ is a complete, lower «-gap chain in $G$ containing $b$. By (i) and the above argument, there is a complete, lower «-gap chain $C$ in $G$ that down-extends $S$ and $\mathfrak{p}=\wedge C=\wedge G$.

Let $\gamma$ be an ordinal number. A set $\left(x_{\lambda}\right)_{1 \leq \lambda \leq \gamma}$ in $Q$ is a descending (respectively, an ascending) $\ll$-series from $a$ to $b$ if

$$
x_{\lambda+1} \ll x_{\lambda} \text { for } \lambda<\gamma, x_{\beta}=\wedge_{\lambda<\beta}\left(x_{\lambda}\right) \text { for limit ordinals } \beta, x_{1}=b, x_{\gamma}=a ;
$$

respectively, $x_{\lambda} \ll x_{\lambda+1}$ for $\lambda<\gamma, x_{\beta}=\vee_{\lambda<\beta}\left(x_{\lambda}\right)$ for limit ordinals $\beta, x_{1}=a, x_{\gamma}=b$.

Proposition 4.8 Let $\ll \in \operatorname{Rel}(Q, \leq)$ and $C$ be a complete chain in $Q$ from a to $b$. Then

(i) $C$ is an upper «-gap chain if and only if it is an ascending $\ll-s e r i e s$.

(ii) $C$ is a lower «-gap chain if and only if it is a descending «-series. 
Proof. (i) Any ascending «-series $\left(x_{\lambda}\right)_{1 \leq \lambda \leq \gamma}$ is, clearly, a $\vee$-complete, upper $\ll$-gap chain. By dual to Lemma 4.5(iii), it is complete. Conversely, let $C$ be a complete, upper «-gap chain from $a$ to $b$. Let $\varnothing \neq G \neq\{b\}$ be any subset of $C$. Then $\wedge G \in C$, as $C$ is complete, and $\wedge G \neq b$. Hence there is $d \in C$ such that $[\wedge G, d] \cap C$ is a gap. So $\wedge G \in G$.

Suppose that, for some ordinal $\beta$,

$$
C=C_{\beta} \cup G_{\beta}, G_{\beta} \cap C_{\beta}=\varnothing \text { and } \vee C_{\beta} \leq \wedge G_{\beta} \text {, }
$$

where $C_{\beta}=\left(c_{\alpha}\right)_{\alpha<\beta}$ is an ascending «-series. Let $G_{\beta} \neq \varnothing$. If there is $\alpha_{0}$ such that $\beta=\alpha_{0}+1$ then $c_{\alpha_{0}}$ is the largest element in $C_{\beta}$. As $C$ is an upper $\ll$-gap chain, take $c_{\beta}=c_{\alpha_{0}+1}$ equal to an immediate «-successor of $c_{\alpha_{0}}$. Clearly, $c_{\beta}$ belongs to $G_{\beta}$. If such $\alpha_{0}$ does not exist then $\beta$ is a limit ordinal. Set $c_{\beta}=\wedge G_{\beta}$. By the above, $c_{\beta} \in G_{\beta}$ and $c_{\alpha}<c_{\beta}$ for all $\alpha<\beta$. In both cases, extend $C_{\beta}$ to the ascending «-series $C_{\beta+1}=\left(c_{\alpha}\right)_{\alpha<\beta+1}$ and restrict $G_{\beta}$ to $G_{\beta+1}=G_{\beta} \backslash\left\{c_{\beta}\right\}$. Then $C=C_{\beta+1} \cup G_{\beta+1}$.

Let $\gamma$ be a limit ordinal. Suppose that there are $\left\{C_{\beta}, G_{\beta}\right\}, \beta<\gamma$, satisfying (4.5). Set $C_{\gamma}=$ $\cup_{\beta<\gamma} C_{\beta}$ and $G_{\gamma}=\cap_{\beta<\gamma} G_{\beta}$. It is easy to see that $C=C_{\gamma} \cup G_{\gamma}, C_{\gamma} \cap G_{\gamma}=\varnothing$ and $c \leq g$ for all $c \in C_{\gamma}$ and $g \in G_{\gamma}$, so that $C_{\gamma} \leq \wedge G_{\gamma}$. Thus $\left\{C_{\gamma}, G_{\gamma}\right\}$ satisfies (4.5). Moreover, $C_{\gamma}$ is an ascending «-series. Thus, by transfinite induction, (4.5) holds for all ordinals. As $C$ is a set, find an ordinal $\gamma$ such that $G_{\gamma}=\varnothing$. Then $C=C_{\gamma}$ is an ascending «-series and $b=\vee C=a_{\gamma}$. The proof of (ii) is similar.

For each $\ll \in \operatorname{Ref}(Q)$, define the reflexive relations $\ll^{\triangleleft}$ and $\ll^{\triangleright}$ from $\operatorname{Ref}(Q)$ :

$a \ll^{\triangleleft} b$ if $a<b$ and there is a complete lower $\ll$-gap chain from $a$ to $b$; $a \ll^{\triangleright} b$ if $a<b$ and there is a complete upper $\ll$-gap chain from $a$ to $b$.

If $a \ll b$ then $\{a, b\}$ is a complete, lower and upper $\ll_{\text {-gap }}$ chain, i.e., $a \ll^{\triangleleft} b$ and $a \ll^{\triangleright} b$. Thus

$$
\ll \subseteq \ll^{\triangleleft} \text { and } \ll \subseteq \ll^{\triangleright}(\text { see }(1.1)) \text {. }
$$

Theorem 4.9 (i) Let $\ll$ be a relation from $\operatorname{Ref}(Q)$. Then $\ll^{\triangleleft}$ and $\ll^{\triangleright}$ are orders,

$$
\ll^{\mathrm{lo}} \subseteq \ll^{\triangleleft}=\left(\ll^{\triangleleft}\right)^{\triangleleft} \text { and } \vec{\ll}=\vec{\ll}^{\triangleleft} ; \quad \ll^{\mathrm{up}} \subseteq \ll^{\triangleright}=\left(\ll^{\triangleright}\right)^{\triangleright} \text { and } \overleftarrow{\ll}=\overleftarrow{\ll^{\triangleright}} \text {. }
$$

(ii) If $\ll$ is a down-expanded order, $\ll=\ll^{\triangleleft}$. If $\ll$ is a dual $\mathbf{T}$-order then $\ll=\ll^{\triangleleft}=\ll^{\text {lo }}$.

(iii) If $\ll$ is an up-expanded order then $\ll=\ll^{\triangleright}$. If $\ll$ is a $\mathbf{T}$-order then $\ll=\ll^{\triangleright}=\ll^{\text {up }}$.

Proof. (i) Clearly, $\ll^{\triangleleft}$ and $\ll^{\triangleright}$ are transitive, so they are orders.

If $a \ll^{\text {lo }} b$ then $[a, b]$ is a complete lower $\ll$-set. Hence, by Proposition 4.7 , there is a complete, lower $\ll$-gap chain from $a$ to $b$. Thus $a \ll^{\triangleleft} b$, so that $\ll^{\text {lo }} \subseteq \ll^{\triangleleft}$.

By (4.7), $\ll^{\triangleleft} \subseteq\left(\ll^{\triangleleft}\right)^{\triangleleft}$. Conversely, let $a\left(\ll^{\triangleleft}\right)^{\triangleleft} b, a \neq b$. Then there is a descending $\ll^{\triangleleft}$ series $\left(x_{\alpha}\right)_{1 \leq \alpha \leq \gamma}$ such that $x_{1}=b$ and $x_{\gamma}=a$. For each ordinal $\alpha, x_{\alpha+1} \ll^{\triangleleft} x_{\alpha}$, so that there is a descending $\ll$-series $\left(u_{\alpha \beta}\right)_{1 \leq \beta \leq \gamma(\alpha)}$ such that $u_{\alpha 1}=x_{\alpha}$ and $u_{\alpha \gamma(\alpha)}=x_{\alpha+1}$. Renumbering $\cup_{1 \leq \alpha \leq \gamma}\left(u_{\alpha \beta}\right)_{1 \leq \beta \leq \gamma(\alpha)}$, we get a descending $\ll$-series $\left(v_{\lambda}\right)_{1 \leq \lambda \leq \delta}$ such that $v_{1}=b$ and $v_{\delta}=a$. This shows that $\left(\ll^{\triangleleft}\right)^{\triangleleft} \subseteq \ll^{\triangleleft}$. So $\left(\ll^{\triangleleft}\right)^{\triangleleft}=\ll^{\triangleleft}$.

As $\ll \subseteq \ll^{\triangleleft}$ by $(4.7)$, we have $\overrightarrow{\ll^{\triangleleft}} \subseteq \gtrless$ by Proposition $4.1($ iii). 
Let $a \gtrless b$. Suppose that $x \ll^{\triangleleft} b$ for some $x \in[a, b)$. By (4.6), there is a complete lower $\ll$-gap chain $C$ from $x$ to $b$. Hence, by Definition $4.4, b$ has an immediate «-predecessor $p \in C$ : $a \leq x \leq p<b$ and $p \ll b$. This contradicts $a \gtreqless b$. So there is no $x \in[a, b)$ such that $x \ll^{\triangleleft} b$, i.e., $a$ $\overrightarrow{\ll^{4}}$ b. Hence $\vec{\ll} \subseteq \overrightarrow{\ll^{4}}$ whence $\vec{\ll}=\overrightarrow{\ll^{4}}$ which completes the proof of the first part of $(4.8)$.

The proof of the first part of (4.8) is similar.

(ii) By (4.7), $\ll \subseteq \ll^{\triangleleft}$. Let $a \ll^{\triangleleft} b$. By (4.6) and Proposition 4.8, there is a descending $\ll$-series $\left(x_{\lambda}\right)_{1 \leq \lambda<\gamma}$ from $a$ to $b: x_{\lambda+1}<x_{\lambda}, x_{\lambda+1} \ll x_{\lambda}$ for $\lambda<\gamma, x_{\beta}=\wedge_{\lambda<\beta}\left(x_{\lambda}\right)$ for limit ordinals $\beta, x_{1}=b$, $x_{\gamma}=a$. Suppose that $x_{\lambda} \ll b$ for some $\lambda$. Then $x_{\lambda+1} \ll x_{\lambda} \ll b$. As $\ll$ is an order, $x_{\lambda+1} \ll b$. Let $\beta$ be a limit ordinal and $x_{\lambda} \ll b$ for all $\lambda<\beta$. As $\ll$ is down-expanded, $x_{\beta}=\wedge_{\lambda<\beta}\left(x_{\lambda}\right) \ll b$. By transfinite induction, $a=x_{\gamma} \ll b$. Thus $\ll^{\triangleleft} \subseteq \ll$ whence $\ll=\ll^{\triangleleft}$.

If $\ll$ is a dual $\mathbf{T}$-order, it is down-contiguous. Hence $\ll \subseteq \ll^{\text {lo }}$ by Proposition 4.3. By (4.8), $\ll^{\text {lo }} \subseteq \ll^{\triangleleft}$. As $\ll=\ll^{\triangleleft}$, we have $\ll=\ll^{\text {lo }}=\ll^{\triangleleft}$.

Part (iii) is proved by duality.

Even if $\ll$ is contiguous, the relations $\ll^{\triangleright}$ and $\ll^{\triangleleft}$ are not necessarily contiguous.

Example 4.10 Let $Q=\{\mathbf{0}, a, b, \mathbf{1}\}, \mathbf{0}<a<\mathbf{1}, \mathbf{0}<b<\mathbf{1}$. Let $\ll$ be a reflexive relation in $Q$, $\mathbf{0} \ll a \ll \mathbf{1}$ and $\mathbf{0} \ll \mathbf{1}$. Then $\ll$ is contiguous, while $\ll^{\triangleright}$ and $\ll^{\triangleleft}$ are not contiguous.

Indeed, $\mathbf{0} \ll^{\triangleright} \mathbf{1}$. However, $[\mathbf{0}, \mathbf{1}] \nsubseteq\left[\ll^{\triangleright}, \mathbf{1}\right]$ and $[\mathbf{0}, \mathbf{1}] \nsubseteq\left[\mathbf{0}, \ll^{\triangleright}\right]$, since $b \in[\mathbf{0}, \mathbf{1}], \mathbf{0} \ll^{\triangleright} b$ and $b K^{\triangleright} \mathbf{1}$. Thus $\ll^{\triangleright}$ is not contiguous. Similarly, $\ll^{\triangleleft}$ is not contiguous.

If, however, $Q$ is a complete chain and $\ll$ is contiguous then $\ll^{\triangleright}$ and $\ll^{\triangleleft}$ are contiguous.

Lemma 4.11 Let $Q$ be a complete chain. If $\ll$ from $\operatorname{Ref}(Q)$ is up-contiguous then the relations $\ll^{\triangleright}, \ll^{\triangleleft}$ are up-contiguous. If $\ll$ is down-contiguous, $\ll^{\triangleright}, \ll^{\triangleleft}$ are down-contiguous.

Proof. Let $a \ll^{\triangleright} b$ and let $T$ be a complete upper $\ll$-gap chain from $a$ to $b$. Then $a \in\left[\ll^{\triangleright}, b\right]$. Let $x \in(a, b]$. Set $c=\vee\{t \in T: t \leq x\}$ and $c^{\prime}=\wedge\{t \in T: x \leq t\}$. As $T$ is complete, $c, c^{\prime} \in T$ and $c \leq x \leq c^{\prime}$. If there is $r \in T$ such that $c<r<c^{\prime}$, then either $x \leq r$, or $r<x$, as $Q$ is a chain. If $x \leq r$ then $c^{\prime} \leq r$, a contradiction. Similarly, the condition $r<x$ gives a contradiction. Thus $\left[c, c^{\prime}\right]_{T}$ is a gap, so that $c^{\prime}=c_{s}$ is the immediate $\ll$-successor of $c$ in $T$.

Let $\ll$ be up-contiguous. Then $c \ll c_{s}$ and $c \leq x \leq c_{s}$ imply $x \ll c_{s}$. Hence $\{x\} \cup\{t \in T$ : $\left.c_{s} \leq t\right\}$ is a complete upper $\ll$-gap chain from $x$ to $b$. Thus $x \ll^{\triangleright} b$ and $\ll^{\triangleright}$ is up-contiguous.

If $\ll$ is down-contiguous then $c \ll c_{s}$ and $c \leq x \leq c_{s}$ imply $c \ll x$. Hence $\{t \in T: t \leq c\} \cup\{x\}$ is a complete upper «-gap chain from $a$ to $x$. Thus $a \ll^{\triangleright} x$ and $\ll^{\triangleright}$ is down-contiguous.

Similarly, if $\ll$ be down-contiguous then $\ll^{\triangleright}, \ll^{\triangleleft}$ are down-contiguous.

By Theorem 4.9, if $\ll$ is a down-expanded order then $\ll^{\triangleleft}=\ll$ is down-expanded. If, however, $\ll$ is not down-expanded, $\ll^{\triangleleft}$ is not necessarily down-expanded even if $Q$ is a complete chain.

Example 4.12 Let $Q$ be a complete chain. If $\ll$ is not down-expanded then $\ll^{\triangleleft}$ is not necessarily down-expanded. If $\ll$ is not up-expanded then $\ll^{\triangleright}$ is not necessarily up-expanded.

Indeed, let $Q=0 \cup\left\{\frac{1}{n}\right\}_{n=1}^{\infty}$ be a subset of $[0,1]$ with usual order $\leq$. Then $(Q, \leq)$ is a complete chain. Let $\ll$ be a reflexive relation in $Q$ such that only $\frac{1}{n} \ll 1$ for all $n=1,2, \ldots$ Then

$$
\ll \text { is an order, } \ll=\ll^{\triangleleft}=\ll^{\triangleright} \text { and }\left[\ll^{\triangleleft}, 1\right]=[\ll, 1]=\{1 / n\}_{n=1}^{\infty} \text {. }
$$

Hence $\wedge\left[\ll^{\triangleleft}, 1\right]=0 \notin\left[\ll^{\triangleleft}, 1\right]$. Thus $\left[\ll^{\triangleleft}, 1\right]$ is not $\wedge$-complete. So $\ll, \ll^{\triangleleft}$ are not down-expanded.

Similar example shows that if $\ll$ is not up-expanded, $\ll^{\triangleleft}$ is not necessarily up-expanded. 
While a relation $\ll$ may have neither $\ll$-radicals, nor dual $\ll$-radicals, the relation $\ll^{\triangleright}$ always has $\ll^{\triangleright}$-radicals and the relation $\ll^{\triangleleft}$ always has dual $\ll^{\triangleleft}$-radicals in all $[a, b] \subseteq Q$.

Corollary 4.13 For $\ll \in \operatorname{Ref}(Q)$, each interval $[a, b] \subseteq Q$ has dual $\ll^{\triangleleft}$-radicals and $\ll^{\triangleright}$-radicals.

Proof. The chain $S=\{b\}$ is a complete, lower «-gap chain in $[a, b]$ containing $b$. It follows from Proposition 4.7 that there is a complete, lower «-gap chain $C$ in $[a, b]$ that down-extends $S$ and $[a, b] \cap[\ll, \mathfrak{p}]=\{\mathfrak{p}\}$, where $\mathfrak{p}=\wedge C$. Hence $a \gtrless \mathfrak{p} \ll^{\triangleleft} b$ and $[a, \mathfrak{p}] \cap[\ll, \mathfrak{p}]=\{\mathfrak{p}\}$ by (2.3). By $(4.8), \vec{\ll}=\overrightarrow{\ll^{\triangleleft}}$. So $a \overrightarrow{\ll^{\triangleleft}} \mathfrak{p}$ and $\mathfrak{p}$ is a dual $\ll^{\triangleleft}$-radical in $[a, b]$. Similarly, $\ll^{\triangleright}$-radicals exist.

For the uniqueness of the radicals in Corollary 4.13 we need some extra conditions.

\section{$5 \quad$ H-relations $\ll$ and the corresponding $\ll^{\triangleleft}$ and $\ll^{\triangleright}$ relations.}

We constructed above the relations $\ll^{\text {lo }}, \ll^{\text {up }}, \ll^{\triangleleft}$ and $\ll^{\triangleright}$. In this section we show that, if $\ll$ is an $\mathbf{H}$-relation then $\ll^{\mathrm{up}}=\ll^{\triangleright}$ is an $\mathbf{R}$-order; if $\ll$ is a dual $\mathbf{H}$-relation, $\ll^{\mathrm{lo}}=\ll^{\triangleleft}$ is a dual $\mathbf{R}$-order. The presence of upper (lower) «-chains effects the structure of lattices.

Proposition 5.1 Let $\ll$ be a dual $\mathbf{H}$-relation in $Q$. Let $C$ be a $\wedge$-complete, lower $\ll$-chain in $a$ $\wedge$-complete set $G \subseteq Q$. Let $b=\vee C \in C$ and $a=\wedge C$. Then

(i) For each $z \in[\mathbf{0}, b] \backslash[\mathbf{0}, a]$, there is $c \in C$ such that $z \neq c \wedge z$ and $c \wedge z \ll z$. In particular, the sets $[\mathbf{0}, b] \backslash[\mathbf{0}, a]$ and $[a, b]$ are lower $\ll-$ sets.

(ii) Each chain in $[a, b]$ larger than $C$ is a lower «-chain.

(iii) There is a maximal chain $S$ in $G \cap[a, b]$ containing $C$; it is a $\wedge$-complete, lower «-chain. If $G$ is complete, $S$ is complete.

Proof. (i) Let $M=\{y \in C: z \leq y\}$. Then $M \neq \varnothing$, as $b \in M$. Let $d=\wedge M$. Then $z \leq d \in C$, since $C$ is $\wedge$-complete, and $a<d$, as $z \notin[\mathbf{0}, a]$. Hence there is $c \in C$ such that $d \neq c \ll d$. Then $c \notin M$. By Lemma 3.1, $c \wedge z \ll d \wedge z=z$. Moreover, $c \wedge z \neq z$, since otherwise $z \leq c$ and $c \in M$. Thus $[\mathbf{0}, b] \backslash[\mathbf{0}, a]$ is a lower $\ll$-set.

If $z \in[a, b]$ then $c \wedge z \in[a, b]$. So $[a, b]$ is a lower $\ll$-set.

(ii) Let $D$ be a chain in $[a, b], C \subset D$ and $a \neq z \in D \backslash C$. By (i), there is $c \in C$ such that $z \neq c \wedge z \ll z$. As $D$ is a chain, $c=c \wedge z$. Thus $z \neq c \ll z$, so that $D$ is a lower $\ll$-chain.

(iii) By Lemma 4.5, $S$ exists and it is $\wedge$-complete. If $G$ is complete, $S$ is complete. By (ii), $S$ is a lower «-chain.

Let $G$ be a $\wedge$-complete set in $Q$ and $b:=\vee G \in G$. It follows from Proposition 4.7 that, for different complete, lower «-gap chains $S$ in $G$ containing $b$ (for example, $S=\{b\}$ ), there are maximal down-extended complete, lower «-gap chains $C_{S}$ in $G$ that down-extend $S$ and the

elements $\mathfrak{p}_{C_{S}}=\wedge C_{S}$ satisfy (4.3). We will show now that if $\ll$ is a dual H-relation then all $\mathfrak{p}_{C_{S}}$ coincide.

Theorem 5.2 Let $G$ be $a \wedge$-complete set in $Q, b:=\vee G \in G$ and $\ll$ be a dual $\mathbf{H}$-relation. Then

(i) There is a unique $\mathfrak{p} \in G$ satisfying $G \cap[\ll, \mathfrak{p}]=\{\mathfrak{p}\}$.

(ii) If $S$ is a complete, lower $\ll-$ gap chain in $G$ and $b \in S$ then $\mathfrak{p} \leq \wedge S$. If $\mathfrak{p}<\wedge S$, there is a 
maximal down-extended complete lower $\ll-$ gap chain $C \subseteq G$ down-extending $S, \mathfrak{p}=\wedge C$.

(iii) The following conditions are equivalent.

1) $G$ is a lower «-set;

2) There is a $\wedge$-complete, lower $\ll$-chain $C$ in $G$ from $a$ to $b$ and $b \in C$;

3) There is a complete, lower $\ll-g a p$ chain $S$ in $G$ from $a$ to $b$ and $b \in S$.

Proof. (i) and (ii). Let $S$ and $T$ be complete, lower «-gap chains in $G$ containing $b$ (for example, $S=\{b\}$ ). By Proposition 4.7, there is a maximal down-extended complete, lower «-gap chain $C$ in $G$ that down-extends $S$ and $\mathfrak{p}_{C}=\wedge C$ satisfies

$$
G \cap\left[\ll, \mathfrak{p}_{C}\right]=\left\{\mathfrak{p}_{C}\right\} .
$$

Let $D$ be another maximal down-extended complete, lower «-gap chain in $G$ that either downextends $S$, or $T$. Set $\mathfrak{p}_{D}=\wedge D$. If $\mathfrak{p}_{C} \notin\left[\mathbf{0}, \mathfrak{p}_{D}\right]$ then, as $\ll$ is a dual $\mathbf{H}$-relation, it follows from Proposition 5.1(i) that there is $z \in D \subseteq G$ such that $\mathfrak{p}_{C} \neq z \wedge \mathfrak{p}_{C} \ll \mathfrak{p}_{C}$ which contradicts (5.1). Thus $\mathfrak{p}_{C} \in\left[\mathbf{0}, \mathfrak{p}_{D}\right]$. Similarly, $\mathfrak{p}_{D} \in\left[\mathbf{0}, \mathfrak{p}_{C}\right]$ whence $\mathfrak{p}_{D}=\mathfrak{p}_{C}$. Thus $\mathfrak{p}_{C}$ is uniquely defined.

(iii) As $\ll$ is a dual H-relation, 2) $\Rightarrow 1$ ) follows from Proposition 5.1(i).

$3) \Rightarrow 2$ ) is evident. As $G$ is a lower $\ll$-set, 1$) \Rightarrow 3$ ) follows from Proposition 4.7.

The following corollary gives an analogue of Theorem 5.2(i) and (ii) for lower «-chains.

Corollary 5.3 Let $G, b, \ll$ and $\mathfrak{p}$ be as in Theorem 5.2. If $T \subseteq G$ is a $\wedge$-complete, lower $\ll$-chain containing $b$ then $\mathfrak{p} \leq \wedge T$. If $\mathfrak{p}<\wedge T$, there is a maximal down-extended $\wedge$-complete lower $\ll$-chain $\mathcal{T}$ in $G$ from $\mathfrak{p}$ to $b$ that down-extends $T$ and $\wedge \mathcal{T}=\mathfrak{p}$.

Proof. Set $t=\wedge T \in T \subseteq G$. As $\ll$ is a dual $\mathbf{H}$-relation, it follows from Theorem 5.2 (iii) that there is a complete, lower «-gap chain $S$ in $G$ from $t$ to $b$. By Theorem 5.2(ii), $\mathfrak{p} \leq t$. If $\mathfrak{p}<t$ then there is a complete, lower «-gap chain $C$ in $G$ from $\mathfrak{p}$ to $b$ that down-extends $S$. Then $T^{\prime}=C \cap[\mathfrak{p}, t]$ is a complete, lower «-gap chain from $\mathfrak{p}$ to $t$. Hence $\mathcal{T}=T^{\prime} \cup T$ is a $\wedge$-complete, lower «-chain in $G$ from $\mathfrak{p}$ to $b$ that down-extends $T$. As $\wedge \mathcal{T}=\mathfrak{p}, \mathcal{T}$ can not be down-extended. So $\mathcal{T}$ is maximal down-extended.

The results analogous to Theorem 5.2 and Corollary 5.3 hold by duality for upper «-chains and upper $\ll$-gap chains if $\ll$ is an $\mathbf{H}$-relation.

To refine the results of Theorem 4.9 for $\mathbf{H}$-relations, we define for each $a \in Q$, the maps $\curlyvee_{a}, \curlywedge_{a}$ on $Q$ by setting

$$
\curlyvee_{a}(x)=a \vee x \text { and } \curlywedge_{a}(x)=a \wedge x \text { for } x \in Q \text {. }
$$

For $G \subseteq Q$, let $\curlyvee_{a}(G)=\left\{\curlyvee_{a}(x): x \in G\right\}$ and $\curlywedge_{a}(G)=\left\{\curlywedge_{a}(x): x \in G\right\}$. Then

$$
\curlywedge_{a}(\wedge G)=\wedge \curlywedge_{a}(G) \text { and } \curlyvee_{a}(\vee G)=\vee \curlyvee_{a}(G) \text {. }
$$

For example, $\curlywedge_{a}(\wedge G) \leq \curlywedge_{a}(g)$ for $g \in G$. So $\curlywedge_{a}(\wedge G) \leq \wedge \curlywedge_{a}(G)$. Conversely, $\wedge\left(\curlywedge_{a}(G)\right) \leq g$ for $g \in G$, so that $\wedge\left(\curlywedge_{a}(G)\right) \leq \wedge G$. As $\wedge\left(\curlywedge_{a}(G)\right) \leq a$, we have $\wedge\left(\curlywedge_{a}(G)\right) \leq \curlywedge_{a}(\wedge G)$. Thus $\curlywedge_{a}(\wedge G)=\wedge \curlywedge_{a}(G)$.

We also have (see [Sk, Theorem 1.4]) that

$$
\text { if } G=\cup_{\alpha \in A} G_{\alpha} \text { then } \wedge G=\wedge\left\{\wedge G_{\alpha}: \alpha \in A\right\} \text { and } \vee G=\vee\left\{\vee G_{\alpha}: \alpha \in A\right\} \text {. }
$$


Proposition 5.4 Let $\ll$ be a dual $\mathbf{H}$-relation and let $G,\left\{G_{\alpha}\right\}_{\alpha \in A}$ be $\wedge$-complete lower $\ll$-sets.

(i) For each $a \in Q$, the set $\curlywedge_{a}(G)$ is a $\wedge$-complete, lower $\ll$-set.

(ii) Let $b=\vee G_{\alpha} \in G_{\alpha}$ for all $\alpha \in A$. Then the $\wedge$-completion $\left(\cup_{\alpha \in A} G_{\alpha}\right)^{\wedge}$ of $\cup_{\alpha \in A} G_{\alpha}$ (see (2.1)) is a lower $\ll-$ set.

Proof. (i) Let $\wedge \curlywedge_{a}(G)<\curlywedge_{a}(g)$ for some $g \in G$. Set $E_{g}=\left\{z \in G\right.$ : $\left.\curlywedge_{a}(g) \leq \curlywedge_{a}(z)\right\}$. Then $g \in E_{g}$ and $e_{g}:=\wedge E_{g} \in G$, as $G$ is $\wedge$-complete. Moreover, $e_{g} \in E_{g}$ and $\curlywedge_{a}(g)=\curlywedge_{a}\left(e_{g}\right)$, since

$$
\curlywedge_{a}\left(e_{g}\right)=\curlywedge_{a}\left(\wedge E_{g}\right) \stackrel{(5.3)}{=} \wedge\left(\curlywedge_{a}\left(E_{g}\right)\right)=\curlywedge_{a}(g) \text {, as } g \in E_{g} \text { and } \curlywedge_{a}(g) \leq \curlywedge_{a}(z) \text { for } z \in E_{g} .
$$

We have $\curlywedge_{a}(\wedge G) \stackrel{(5.3)}{=} \wedge \curlywedge_{a}(G)<\curlywedge_{a}(g)=\curlywedge_{a}\left(e_{g}\right)$ by above. As $\wedge G \leq e_{g}$, it follows that $\wedge G<e_{g}$. As $G$ is a lower $\ll$-set, there is $h \in G$ such that $h \ll e_{g}$ and $h<e_{g}$. As $e_{g}$ is minimal in $E_{g}, \curlywedge_{a}(h) \neq \curlywedge_{a}(g)$. So $\curlywedge_{a}(h)<\curlywedge_{a}\left(e_{g}\right)=\curlywedge_{a}(g)$. As $\ll$ is a dual H-relation, $\curlywedge_{a}(h)=a \wedge h \ll$ $a \wedge e_{g}=\curlywedge_{a}\left(e_{g}\right)=\curlywedge_{a}(g)$, i.e., $\curlywedge_{a}(h)$ is a $\ll$-predecessor of $\curlywedge_{a}(g)$. Thus $\curlywedge_{a}(G)$ is a lower $\ll$-set.

For $N \subseteq \curlywedge_{a}(G)$, there is $M \subseteq G$ with $N=\curlywedge_{a}(M)$. As $G$ is $\wedge$-complete, $\wedge M \in G$. Hence $\wedge N=\wedge \curlywedge_{a}(M) \stackrel{(5.3)}{=} \curlywedge_{a}(\wedge M) \in \curlywedge_{a}(G)$. Thus $\curlywedge_{a}(G)$ is $\wedge$-complete.

(ii) Set $K=\cup_{\alpha \in A} G_{\alpha}$. Let $x \in K^{\wedge}$ be such that $\wedge K<x$. Then $x=\wedge M$ for some $M \subseteq K$. Let $F_{\alpha}=M \cap G_{\alpha}, N_{\alpha}=G_{\alpha} \backslash F_{\alpha}$ and $n_{\alpha}=\wedge N_{\alpha}$ for all $\alpha \in A$. Then $K=\left(\cup_{\alpha} N_{\alpha}\right) \cup M$ and

$$
\wedge K \stackrel{(5.4)}{=}\left(\wedge\left\{n_{\alpha}: \alpha \in A\right\}\right) \wedge x=\wedge\left\{n_{\alpha} \wedge x: \alpha \in A\right\} .
$$

If $n_{\alpha} \wedge x=x$ for all $\alpha$, then $\wedge K=x-$ a contradiction. Thus $n_{\beta} \wedge x<x$ for some $\beta$. As $N_{\beta} \subseteq G_{\beta}$, we have $\wedge \curlywedge_{x}\left(G_{\beta}\right)=\curlywedge_{x}\left(\wedge G_{\beta}\right) \leq n_{\beta} \wedge x<x$. As $x \leq b \in G_{\beta}$, we have $x=\curlywedge_{x}(b) \in \curlywedge_{x}\left(G_{\beta}\right)$. By (i), $\curlywedge_{x}\left(G_{\beta}\right)$ is a $\wedge$-complete, lower «-set. Hence there is $u \in G_{\beta}$ such that $\curlywedge_{x}(u) \ll \curlywedge_{x}(b)$ and $\curlywedge_{x}(u) \neq \curlywedge_{x}(b)$, i.e., $x \neq x \wedge u \ll x$. As $x \wedge u \in K^{\wedge}, K^{\wedge}$ is a lower $\ll$-set.

We will use Proposition 5.4 to prove the main results of this section.

Theorem 5.5 (i) Let $\ll$ be a dual $\mathbf{H}$-relation. Then

$1) \ll^{\triangleleft}=\ll^{\text {lo }}$ is a dual $\mathbf{R}$-order and $\overleftrightarrow{\ll}=\vec{\ll}^{\triangleleft}=(\overleftrightarrow{\ll})^{\triangleright}=(\overleftrightarrow{\ll})^{\text {up }}$ is an $\mathbf{R}$-order;

2) for each $[a, b] \subseteq Q$, there is a unique dual $\ll^{\triangleleft}$-radical $\mathfrak{p}=\mathfrak{p}_{[a, b]}$ such that $a \gtrless \mathfrak{p} \ll^{\triangleleft} b$. Moreover, $a \leq x \ll^{\triangleleft} b$ implies $x \in[\mathfrak{p}, b]$.

(ii) Let $\ll$ be an $\mathbf{H}$-relation and $[a, b] \subseteq Q$. Then

$1) \ll^{\triangleright}=\ll^{\mathrm{up}}$ is an $\mathbf{R}$-order, and $\overleftarrow{\ll}=\overleftarrow{\ll^{\triangleright}}=(\overleftarrow{\ll})^{\triangleleft}=(\overleftarrow{\ll})^{\text {lo }}$ is a dual $\mathbf{R}$-order;

2) for each $[a, b] \subseteq Q$, there is a unique $\ll^{\triangleright}$-radical $\mathfrak{r}=\mathfrak{r}_{[a, b]}$ such that $a \ll^{\triangleright} \mathfrak{r} \overleftarrow{\ll} b$. Moreover, $a \ll^{\triangleright} x \leq b$ implies $x \in[a, \mathfrak{r}]$.

Proof. (i) 1) Let $\ll$ be a dual H-relation. Firstly, let us show that $\ll^{\triangleleft}$ is down-expanded. Let $G=\left[\ll^{\triangleleft}, c\right]$ for some $c \in Q$. For each $g \in G \backslash\{c\}, G_{g}=\{g, c\}$ is a complete, lower $\ll^{\triangleleft}$-set and $G=\cup_{g \in G \backslash\{c\}} G_{g}$. Hence $G^{\wedge}$ is a $\wedge$-complete, lower $\ll^{\triangleleft}$-set by Proposition 5.4. As $\wedge\left(G^{\wedge}\right)=\wedge G$, it follows from Theorem 5.2(iii) that there is a complete lower $\ll^{\triangleleft}$-gap chain $C$ in $G^{\wedge}$ such that $\wedge C=\wedge G$ and $\vee C=c$. Hence $\wedge G\left(\ll^{\triangleleft}\right)^{\triangleleft} c$. As $\ll^{\triangleleft}=\left(\ll^{\triangleleft}\right)^{\triangleleft}$ by Theorem 4.9 , we have $\wedge G \ll^{\triangleleft} c$. Thus $\left[\ll^{\triangleleft}, c\right]$ is $\wedge$-complete whence $\ll^{\triangleleft}$ is down-expanded. 
It follows from Theorem 5.2(iii) that $[a, b]$ is a lower $\ll$-set $\left(a \ll^{\text {lo }} b\right.$ (see Definition 4.2)) if and only if $a \ll^{\triangleleft} b$. Thus $\ll^{\triangleleft}=\ll^{\text {lo }}$ is down-expanded.

Let us now prove that $\rightleftarrows$ is an $\mathbf{H}$-order. Suppose that $a \gtrless b$ and $a \leq c$. Assume that $c \leq x \ll b \vee c$ for some $x$. As $\ll$ is a dual H-relation, $a \leq x \wedge b \ll(b \vee c) \wedge b=b$. As $a \gtrless b$, we have $x \wedge b=b$ by (2.3). So $b \leq x$. Thus $x=b \vee c$. From this we conclude that $c \ll b \vee c$. It follows from Lemma 3.1 that $\gtrless$ is an $\mathbf{H}$-relation.

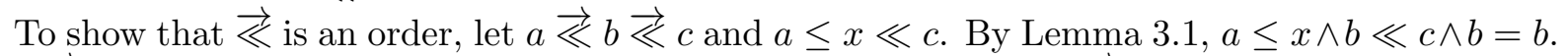

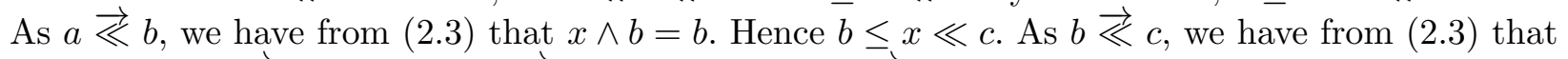

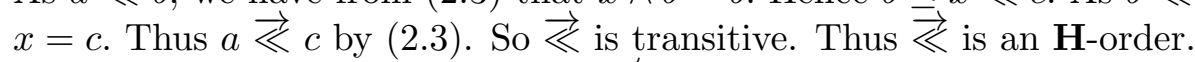

Similarly, if $\ll$ is an $\mathbf{H}$-relation, $\overleftarrow{\ll}$ is a dual $\mathbf{H}$-order. Hence if $\ll$ is a dual $\mathbf{H}$-relation then $\overleftarrow{(\vec{\ll})}$ is a dual H-order. By Proposition $4.3, \ll^{\mathrm{lo}}=\overleftarrow{(\vec{\ll})}$ whence $\ll^{\text {lo }}$ is a dual $\mathbf{H}$-order. Combining this with the fact that $\ll^{\triangleleft}=\ll^{\text {lo }}$ is down-expanded, we get that $\ll^{\triangleleft}=\ll^{\text {lo }}$ is a dual $\mathbf{R}$-order.

By duality, $\ll^{\triangleright}=\ll^{\text {up }}$ is an $\mathbf{R}$-order, if $\ll$ is an $\mathbf{H}$-relation. Hence, as $\ll$ is an $\mathbf{H}$-order by above, $(\vec{\ll})^{\triangleright}=(\vec{\ll})^{\text {up }}$. Therefore, since $\ll^{\mathrm{lo}}=\overleftarrow{(\overleftrightarrow{\ll})}$ and $\ll^{\mathrm{up}}=\overrightarrow{(\overleftarrow{\ll})}$ by Proposition 4.3 ,

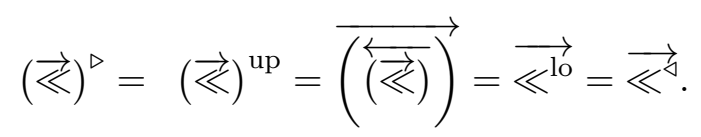

By (4.7) and Proposition 4.1, $\overrightarrow{\ll^{\triangleleft}} \subseteq \vec{\ll}$. By Proposition 4.3, $\vec{\ll} \subseteq \overrightarrow{\ll^{\text {lo }}}$. As $\ll^{\triangleleft}=\ll^{\text {lo }}$, we have $\overrightarrow{\ll^{\triangleleft}}$ $\subseteq \gtrless \subseteq \ll^{\triangleleft}$. So $\vec{\ll}=\overrightarrow{\ll^{\natural}}$. By $(5.5), \vec{\ll}=(\vec{\ll})^{\triangleright}$. So, as $\vec{\ll}$ is an H-order, $(\vec{\ll})^{\triangleright}$ is an $\mathbf{R}$-order by above. Thus $\gtrless$ is an $\mathbf{R}$-order. The proof of 1 ) is complete.

2) As $\ll^{\triangleleft}$ is a dual $\mathbf{R}$-order, it is a dual $\mathbf{T}$-order. By Theorem 2.4 and (2.5), for each $[a, b] \subseteq Q$ there is a unique dual $\ll^{\triangleleft}$-radical $\mathfrak{p} \in[a, b]$ such that $a \overrightarrow{\ll^{\triangleleft}} \mathfrak{p} \ll^{\triangleleft} b$. As $\overrightarrow{\ll^{\triangleleft}}=\overleftrightarrow{\ll}$ by 1$), a \overleftrightarrow{\ll} \mathfrak{p} \ll^{\triangleleft} b$.

Let $a \leq x \ll^{\triangleleft} b$. As $\ll^{\triangleleft}$ is a dual R-order, $a \leq x \wedge \mathfrak{p} \ll^{\triangleleft} b \wedge \mathfrak{p}=\mathfrak{p}$ by Lemma 3.1(ii). As $a \overleftrightarrow{\ll^{\triangleleft}}$ $\mathfrak{p}$ by above, we have from (2.3) that $x \wedge \mathfrak{p}=\mathfrak{p}$. Hence $\mathfrak{p} \leq x$.

The proof of part (ii) is similar.

The following corollary strengthens Theorem 2.5

Corollary 5.6 Let $\ll$ be a dual $\mathbf{H}$-relation. The following conditions are equivalent.

(i) $\ll$ is a dual $\mathbf{R}$-order. $\quad$ (ii) $\ll=\ll^{\triangleleft}$.

(iii) $\ll$ is an order and $\wedge_{n \in \mathbb{N}}\left(x_{n}\right) \ll x_{1}$ for each descending $\ll-$ series $\left(x_{n}\right)_{n \in \mathbb{N}}$.

(iv) For each $[a, b] \subseteq Q$, there is $c \in[a, b]$ such that $a \gtreqless c \leq b$.

(v) Each $[a, b] \subseteq Q$ has a unique dual «-radical.

Proof. (ii) $\Rightarrow$ (i) As $\ll$ is a dual $\mathbf{H}$-relation, $\ll^{\triangleleft}$ is a dual $\mathbf{R}$-order by Theorem 5.5 . So $\ll$ is a dual R-order.

(i) $\Rightarrow$ (iii). As $x_{n+1} \ll x_{n}$ for all $n$, and $\ll$ is transitive, $x_{n} \ll x_{1}$. As $\ll$ is down-expanded by Definition $3.2, \wedge\left(x_{n}\right)_{n \in \mathbb{N}} \ll x_{1}$.

(iii) $\Rightarrow$ (ii). If $a \ll^{\triangleleft} b$, there is a descending $\ll$-series $\left(x_{\alpha}\right)_{1 \leq \alpha \leq \gamma}$ with $a=x_{\gamma}$ and $b=x_{1}$. If $x_{\alpha} \ll b$ for some $\alpha$, then $x_{\alpha+1} \ll x_{\alpha} \ll b$ implies $x_{\alpha+1} \ll b$ by transitivity of $\ll$. Hence all $x_{\alpha+n} \ll b$. If $\beta$ is a limit ordinal and $\alpha$ is the previous limit ordinal then, $x_{\beta}=\wedge_{n \in \mathbb{N}}\left(x_{\alpha+n}\right) \ll x_{\alpha} \ll b$. So $x_{\beta} \ll b$. Thus, by transfinite induction, $a \ll b$. So $\ll=\ll^{\triangleleft}$. 
(i) $\Rightarrow$ (iv). As dual $\mathbf{R}$-orders are dual $\mathbf{T}$-orders, it follows from Theorem 2.4 (ii) that each $[a, b] \subseteq Q$ has a unique dual «-radical $\mathfrak{p}: a \vec{\ll} \ll b$ (see $(2.5)$ ). If $\mathfrak{p}=a$ then $a \ll b$. If $\mathfrak{p} \neq a$, set $c=\mathfrak{p}$. As $\mathfrak{p} \ll b$ implies $\mathfrak{p} \leq b$, (iv) holds.

(iv) $\Rightarrow$ (ii). Let $a \ll^{\triangleleft} b$ for $a<b$. If $a \nless b$ then, by (iv), $a \gtrless c \leq b$ for some $c \neq a$. On the other hand, by Theorem 5.5, $\ll^{\triangleleft}=\ll^{\text {lo }}$. Thus, by Definition $4.2,[a, b]$ is a lower $\ll$-set, so that there is $d \in[a, b]$ such that $c \neq d \ll c$ which contradicts $a \gtrless c$. Thus $a \ll b$. So $\ll^{\triangleleft} \subseteq \ll$. Hence (4.7) implies $\ll=\ll^{\triangleleft}$.

(i) $\Rightarrow(\mathrm{v})$. As dual R-orders are dual T-orders, it follows from Theorem 2.5 that each $[a, b] \subseteq Q$ has a unique dual «-radical.

$(\mathrm{v}) \Rightarrow$ (iv). If $[a, b] \subseteq Q$ has a dual $\ll$-radical $\mathfrak{p}$ then $a \ll \mathfrak{p} \ll b$ by $(2.5)$. So (iv) holds.

It should be noted that Amitsur [A-I] got some results equivalent to the implication (ii) $\Leftrightarrow$ (iv).

By duality, the following results are equivalent for an $\mathbf{H}$-relation $\ll$ :

(i) $\ll$ is an $\mathbf{R}$-order; (ii) $\ll=\ll^{\triangleright}$; (iii) Each $[a, b] \subseteq Q$ has a unique $\ll$-radical.

If $\ll$ is an $\mathbf{H}$-relation in $Q$, it follows from Theorem 5.5 that $\ll^{\triangleright}$ is an $\mathbf{R}$-order and $\overleftarrow{\ll}$ is a dual $\mathbf{R}$-order in $Q$. Hence each $[a, b] \subseteq Q$ has a unique $\ll^{\triangleright}$-radical and a unique dual $\overleftarrow{\ll}$-radical.

Similarly, if $\ll$ is a dual $\mathbf{H}$-relation in $Q$, then $\ll^{\triangleleft}$ is a dual $\mathbf{R}$-order and $\gtreqless$ is an $\mathbf{R}$-order in $Q$.

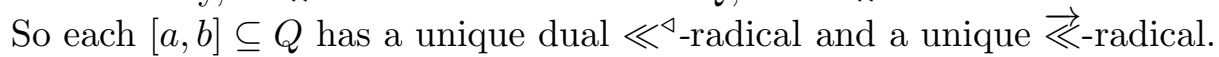

Proposition 5.7 (i) Let $\ll$ be an $\mathbf{H}$-relation. Then, for each $[a, b] \subseteq Q$,

1) the $\ll^{\triangleright}$-radical and the dual $\overleftarrow{\ll}$-radical in $[a, b]$ coincide;

$2)$ if there exists $a \ll$-radical in $[a, b]$, it coincides with the $\ll^{\triangleright}$-radical.

(ii) Let $\ll$ be a dual $\mathbf{H}$-relation. Then, for each $[a, b] \subseteq Q$,

1) the dual $\ll^{\triangleleft}$-radical and the $\vec{\ll}$-radical in $[a, b]$ coincide;

2) if there exists a dual «-radical in $[a, b]$, it coincides with the dual $\ll^{\triangleleft}$-radical.

Proof. (i) 1) By Theorem 5.5(ii), the $\ll^{\triangleright}$-radical $\mathfrak{r}$ satisfies $a \ll^{\triangleright} \mathfrak{r} \overleftarrow{\ll} b$. By (2.5), the dual $\overleftarrow{\ll}$-radical $\mathfrak{p}$ in $[a, b]$ satisfy $a \stackrel{\rightleftarrows}{\ll} \mathfrak{\aleph} b$. By Proposition $4.3, \stackrel{\rightleftarrows}{\ll}=\ll$ up . By Theorem $5.5, \ll^{\text {up }}=$ $\ll^{\triangleright}$, as $\ll$ is an H-relation, So $\stackrel{\rightleftarrows}{\ll}=\ll^{\triangleright}$. Hence $a \stackrel{\rightleftarrows}{\ll} \mathfrak{p} b$ turns into $a \ll \mathfrak{p} \overleftarrow{\ll} b$. Comparing it to $a \ll^{\triangleright} \mathfrak{r} \overleftarrow{\ll} b$ and taking into account the uniqueness of the radicals, we get $\mathfrak{r}=\mathfrak{p}$.

2) If $\mathfrak{r}_{1}$ is the $\ll$-radical in $[a, b]$ then $a \ll \mathfrak{r}_{1} \overleftarrow{\ll} b$. As $\ll \subseteq \ll^{\triangleleft}$ by $(4.7), a \ll^{\triangleleft} \mathfrak{r}_{1} \overleftarrow{\ll} b$. Since (see (i)) $a \ll^{\triangleright} \mathfrak{r} \overleftarrow{\ll} b$, it follows from the uniqueness of the radical that $\mathfrak{r}=\mathfrak{r}_{1}$.

For a subset $F \subseteq \operatorname{Ref}(Q)$, define the relations $\ll_{\wedge F}:=\cap_{\ll \in F} \ll$ and $\ll_{\vee F}:=\cup_{\ll \in F} \ll$ by

$$
a \ll_{\wedge F} b \text { if } a \ll b \text { for all } \ll \text { in } F ; a \ll_{\vee F} b \text { if } a \ll b \text { for some } \ll \text { in } F \text {. }
$$

They belong to $\operatorname{Ref}(Q)$. In particular, for $\prec$ and $\ll, a(\prec \cap \ll) b$ if and only if $a \prec b$ and $a \ll b$.

If $\ll$ is an $\mathbf{H}$-relation and $\prec$ is not an $\mathbf{H}$-relation, then $\prec \cap \ll$ is not necessarily an $\mathbf{H}$-relation.

Lemma 5.8 Let $\ll$ be an $\mathbf{H}$-relation, let $\prec$, $\sqsubset$ be relations in $\operatorname{Ref}(Q)$ and $\ll$ be stronger than $\sqsubset$ (see (1.1)). If $\prec \cap \sqsubset$ is an $\mathbf{H}$-relation then $\prec \cap \ll$ is also an $\mathbf{H}$-relation.

The same is true for dual $\mathbf{H}$-relations. 
Proof. Let $a(\prec \cap \ll) b$ for $a, b \in Q$. Then $a \prec b$ and $a \ll b$. As $\ll$ is stronger than $\sqsubset$, we have $a \sqsubset b$. By (5.6), $a(\prec \cap \sqsubset) b$. Since $\prec \cap \sqsubset$ is an H-relation, $a \vee c(\prec \cap \sqsubset) b \vee c$ for all $c \in Q$, by Definition 3.2. Hence $a \vee c \prec b \vee c$ by (5.6). As $\ll$ is an H-relation, we also have $a \vee c \ll b \vee c$. So $a \vee c(\prec \cap \ll) b \vee c$ by (5.6). Thus $\prec \cap \ll$ is a H-relation (see Definition 3.2).

For convenience, we summarize below some results about $\mathbf{H}$-relations which will be used later.

Theorem 5.9 (i) Let $\ll$ be an $\mathbf{H}$-relation and $a \in Q$. Then $\ll$ is an $\mathbf{R}$-order and

1) $\mathfrak{r}(a)=\vee\left[\ll^{\triangleright}, a\right]$ is the $\ll^{\triangleright}$-radical in $[a, \mathbf{1}]$, i.e., $a \ll^{\triangleright} \mathfrak{r}(a) \overleftarrow{\ll} \mathbf{1}$

2) $\mathfrak{r}(b)=\mathfrak{r}(a)$ for all $b \in[a, \mathfrak{r}(a)]$ - there is an ascending $\ll$-series from $b$ to $\mathfrak{r}(a)$;

3) each $b \in[a, \mathbf{1}] \backslash[\mathfrak{r}(a), \mathbf{1}]$ has $a \ll$-successor $s_{b}: b \ll s_{b} \neq b$; $\mathfrak{r}(a)$ has no $\ll$-successor.

(ii) Let $\ll$ be a dual $\mathbf{H}$-relation and $b \in Q$. Then $\ll^{\triangleleft}$ is a dual $\mathbf{R}$-order and

1) $\mathfrak{p}(b)=\wedge\left[\ll^{\triangleleft}, b\right]$ is the dual $\ll^{\triangleleft}$-radical in $[\mathbf{0}, b] \subseteq Q$, i.e., $\mathbf{0} \ll \mathfrak{p}(b) \ll^{\triangleleft} b$;

2) $\mathfrak{p}(a)=\mathfrak{p}(b)$ for all $a \in[\mathfrak{p}(b), b]$ - there is a descending $\ll$-series from a to $\mathfrak{p}(b)$;

3) each $a \in[\mathbf{0}, b] \backslash[\mathbf{0}, \mathfrak{p}(b)]$ has a $\ll$-predecessor $p_{a} \ll a \neq p_{a} ; \mathfrak{p}(b)$ has no $\ll$-predecessor.

Let $Q$ be a complete lattice. For $a \in Q$, set

$$
\sigma(a)=\wedge[\ll, a] \text { and } s(a)=\vee[a, \ll]
$$

Then if $\ll$ is an $\mathbf{H}$-relation, we have

$$
[a, \ll] \stackrel{(4.7)}{\subseteq}\left[a, \ll^{\triangleright}\right], \text { so that } s(a) \leq \vee\left[a, \ll^{\triangleright}\right] \stackrel{(2.7)}{=} \mathfrak{r}_{\ll^{\triangleright}}(a) .
$$

If $\ll$ is a dual $\mathbf{H}$-relation, we have

$$
[\ll, a] \stackrel{(4.7)}{\subseteq}\left[\ll^{\triangleleft}, a\right], \text { so that } \sigma(a) \geq \wedge\left[\ll^{\triangleleft}, a\right] \stackrel{(2.6)}{=} \mathfrak{p}_{\ll^{\triangleleft}}(a) .
$$

A bijection $\theta: Q \rightarrow Q^{\prime}$ is an isomorphism if $x \leq y \Leftrightarrow \theta(x) \leq \theta(y)$ for all $x, y \in Q$. Then

$$
\theta(\vee G)=\vee \theta(G) \text { and } \theta(\wedge G)=\wedge \theta(G) \text { for each } G \subseteq Q .
$$

Indeed, $\theta(x) \leq \theta(\vee G)$, as $x \leq \vee G$ for $x \in G$. Thus $\vee \theta(G) \leq \theta(\vee G)$. As $\theta^{-1}$ is also an isomorphism, $\vee G=\vee \theta^{-1}(\theta(G)) \leq \theta^{-1}(\vee \theta(G))$. So $\theta(\vee G) \leq \vee \theta(\vee G)$. Hence $\theta(\vee G)=\vee \theta(G)$. Similarly, $\theta(\wedge G)=\wedge \theta(G)$

Let $\theta$ be an automorphism of $Q$. It preserves a relation $\ll$ from $\operatorname{Ref}(Q)$ if

$$
\theta(x) \ll \theta(y) \Leftrightarrow x \ll y \text { for all } x, y \in Q .
$$

For $x<y$ in $Q, \theta$ is an isomorphism from $[x, y]$ onto $[\theta(x), \theta(y)]$. Clearly, $[x, y]$ is a lower (upper) $\ll$-set if and only if $[\theta(x), \theta(y)]$ is a lower (upper) «-set. So $x \ll^{\text {lo }} y$ if and only if $\theta(x) \ll^{\text {lo }} \theta(y)$, and $x \ll^{\text {up }} y$ if and only if $\theta(x) \ll^{\text {up }} \theta(y)$. Thus $\theta$ preserves the relations $\ll^{\text {lo }}$ and $\ll^{\text {up }}$.

Proposition 5.10 Let an automorphism $\theta$ of $Q$ preserve a relation $\ll$ from $\operatorname{Ref}(Q)$.

(i) If $\ll$ is an $\mathbf{H}$-relation, $\theta$ preserves $\ll^{\triangleright}$; if $\ll$ is a dual $\mathbf{H}$-relation, $\theta$ preserves $\ll^{\triangleleft}$.

(ii) Let $\theta(a)=a$ for some $a \in Q$. Then $\theta(\sigma(a))=\sigma(a)$ and $\theta(s(a))=s(a)$. Moreover,

1) if $\ll$ is a $\mathbf{T}$-order then $\theta(\mathfrak{r}(a))=\mathfrak{r}(a)$; if it is a dual $\mathbf{T}$-order then $\theta(\mathfrak{p}(a))=\mathfrak{p}(a)$;

2) if $\ll$ is an $\mathbf{H}$-relation, $\theta\left(\mathfrak{r}_{\ll \triangleright}(a)\right)=\mathfrak{r}_{\ll \triangleright}(a)$; if it is a dual $\mathbf{H}$-relation, $\theta\left(\mathfrak{p}_{\ll \triangleleft}(a)\right)=\mathfrak{p}_{{ }^{\triangleleft}}(a)$. 
Proof. (i) follows, as $\theta$ preserves $\ll^{\text {lo }}$ and $\ll^{\text {up }}$, and as $\ll^{\mathrm{lo}}=\ll^{\triangleleft}$, $\ll^{\mathrm{up}}=\ll^{\triangleright}$ by Theorem 5.5.

(ii) As $\theta$ and $\theta^{-1}$ are automorphisms, $\theta([\ll, a])=[\ll, a]$ and $\theta([a, \ll])=[a, \ll]$. By $(5.10)$,

$$
\theta(\sigma(a))=\theta(\wedge[\ll, a])=\wedge[\ll, a]=\sigma(a) \text { and } \theta(s(a))=\theta(\vee[a, \ll])=\vee[a, \ll]=s(a) \text {. }
$$

By $(2.7), s(a)=\mathfrak{r}(a)$ if $\ll$ is a $\mathbf{T}$-order. By $(2.6), \sigma(a)=\mathfrak{p}(a)$ if $\ll$ is a dual T-order. So 1) follows from (5.12). Part 2) follows from (i) and 1).

\section{Gap $<_{\mathfrak{g}}$ and continuity $<_{\mathfrak{c}}$ relations in complete lattices.}

Definition 6.1 Let $a \leq b$ in $Q$. We write $a<_{\mathfrak{g}} b$ if either $a=b$, or $[a, b]$ is a gap.

We write $a<_{\mathfrak{c}} b$ if either $a=b$, or there is a complete continuous chain $C$ from $a$ to $b$ : For all $x<y$ in $C$, there is $z \in C$ such that $x<z<y$, i.e., $C$ has no gaps.

The relations $<_{\mathfrak{g}}$ and $<_{\mathfrak{c}}$ belong to $\operatorname{Ref}(Q)$ and $<_{\mathfrak{c}}$ is an order.

Proposition 6.2 A complete lattice $Q$ has no gaps if and only if $<_{\mathfrak{c}}=\leq$.

Proof. Clearly, if $<_{\mathfrak{c}}=\leq$ then $Q$ has no gaps. Conversely, let $a<b$. By Lemma 4.5, there is a maximal complete chain $C$ from $a$ to $b$. If $C$ is not continuous, there is a gap $[x, y]_{C}$ in $C$. As $Q$ has no gaps, there is $z \in Q, z \notin C$, with $x<z<y$. Hence the chain $C \cup\{z\}$ is larger than $C$, a contradiction. Thus $C$ is continuous. So $a<_{\mathfrak{c}} b$. Hence $\leq \subseteq<_{\mathfrak{c}}$. As $<_{\mathfrak{c}} \subseteq \leq$, we have $<_{\mathfrak{c}}=\leq$.

Example 6.3 In general, the order $<_{\mathfrak{c}}$ is neither contiguous, nor expanded.

(i) Let $Q=[0,1] \cup\{a\}$, where $[0,1] \subset \mathbb{R}, \mathbf{0}=0, \mathbf{1}=1, a \wedge t=\mathbf{0}$ and $a \vee t=\mathbf{1}$ for $t \in(0,1)$. Then $Q$ is a complete lattice and $\mathbf{0}<_{\mathfrak{c}} \mathbf{1}$. As $a \nless_{\mathfrak{c}} \mathbf{1}$, we have $[\mathbf{0}, \mathbf{1}] \nsubseteq\left[<_{\mathfrak{c}}, \mathbf{1}\right]$. So $<_{\mathfrak{c}}$ is not contiguous.

(ii) Let $Q_{n}, 2 \leq n$, be the interval in $\mathbb{R}^{2}$ from $\left(\frac{1}{n}, \frac{1}{n}\right)$ to $(1,0)$. Let $L=\cup_{n=2}^{\infty} Q_{n}$ and $(x, y) \leq(u, v)$ in $L$ if they lie in the same $Q_{n}$ and $x \leq u$. Then $\mathbf{1}=(1,0)$. Set $Q=(0,0) \cup L$ and $\mathbf{0}=(0,0)<(x, y)$ for all $(x, y) \in L$. Then $Q$ is a complete lattice, $\left[<_{\mathfrak{c}}, \mathbf{1}\right]=L, \mathbf{0}=\wedge\left[<_{\mathfrak{c}}, \mathbf{1}\right]$ and $\mathbf{0} \nless_{\mathfrak{c}} \mathbf{1}$. So $<_{\mathfrak{c}}$ is not expanded.

We need now the following lemma.

Lemma 6.4 Let $T$ be a complete chain from a to $b$. Let each $t \in T \backslash\{b\}$ have an immediate successor $t_{s}$ in $T$, i.e., $\left[t, t_{s}\right]_{T}=\left\{t, t_{s}\right\}$ is a gap, and let $C_{t}$ be some complete chain from $t$ to $t_{s}$. Set $C_{b}=\{b\}$. Then the chain $C=\cup_{t \in T} C_{t}$ is complete.

Proof. Let $G \subseteq C$. For $t \in T$, set $G_{t}=G \cap C_{t}$ and $Y=\left\{t \in T: G_{t} \neq \varnothing\right\}$.

Let $y=\vee Y$ and $c_{t}=\vee G_{t}$ for $t \in Y$. As $T$ and all $C_{t}$ are complete, $y \in T$ and $c_{t} \in C_{t}$. As $G=\cup_{t \in Y} G_{t}$, we have from (5.4) that $\vee G=\vee\left\{c_{t}: t \in Y\right\}$. If $y \in Y$ then $\vee G=c_{y} \in C_{y} \subseteq C$. If $y \notin Y$ then $t<t_{s} \leq y$ for all $t \in Y$, as $\left[t, t_{s}\right]_{T}$ is a gap. So $y=\vee\left\{t_{s}: t \in Y\right\}$. As $t \leq c_{t} \leq t_{s}$, we have $y=\vee Y \leq \vee G=\vee\left\{c_{t}: t \in Y\right\} \leq \vee\left\{t_{s}: t \in Y\right\}=y$. Hence $\vee G=y \in T \subseteq C$. Thus $C$ is $\vee$-complete.

Let $z=\wedge Y$ and $g_{t}=\wedge G_{t}$ for $t \in Y$. As $T$ and all $C_{t}$ are complete, $z \in T$ and $g_{t} \in C_{t}$. As $G=\cup_{t \in Y} G_{t}$, we have from (5.4) that $\wedge G=\wedge\left\{g_{t}: t \in Y\right\}$. If $z \notin Y$ then $z<t$ for all $t \in Y$, and $z=\wedge Y$. As $\left[z, z_{s}\right]_{T}$ is a gap, we get a contradiction. Hence $z \in Y$. Then $G_{z} \neq \varnothing$ and $\wedge G=\wedge G_{z}=g_{z} \in C_{z} \subseteq C$. Thus $C$ is $\wedge$-complete. So $C$ is complete.

Lemma 6.4 also holds if each $t \in T \backslash\{a\}$ has an immediate predecessor $t_{p}$ in $T:\left[t_{p}, t\right]_{T}$ is a gap. 
Proposition 6.5 (i) $<_{\mathfrak{c}}=<_{\mathfrak{c}}^{\triangleleft}=<_{\mathfrak{c}}^{\triangleright}$.

(ii) Each complete continuous chain from $a$ to $b$ in $Q$ is maximal in $[a, b]$.

Proof. (i) Let $a<_{\mathfrak{c}} b$. Then there is a complete upper $<_{\mathfrak{c}}$-gap chain $T$ from $a$ to $b$, i.e., each $t \in T \backslash\{b\}$ has an immediate $<_{\mathfrak{c}}$-successor $t_{s}$ in $T, t<_{\mathfrak{c}} t_{s}$. Then there is a complete continuous

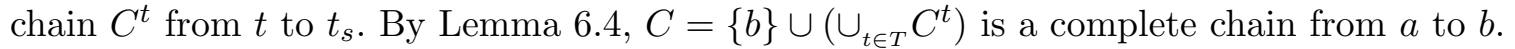

If $C$ is not continuous, there are $x<y$ in $C$ such that $[x, y]_{C}$ is a gap. Let $t \in T$ be such that $x \in C^{t} \backslash\left\{t_{s}\right\}$. If $y \in C^{t}$ then $[x, y]_{C}=[x, y]_{C^{t}}$ is not a gap, as $C^{t}$ is continuous. If $y \notin C^{t}$ then $y \neq t_{s}$ and $y \in C^{u}, t<u \in T$. Hence $x<t_{s}<y$. So $[x, y]_{C}$ is not a gap. Thus $C$ is continuous and $a<_{\mathfrak{c}} b$. So $<_{\mathfrak{c}}^{\triangleright} \subseteq<_{\mathfrak{c}}$. As also $<_{\mathfrak{c}} \subseteq<_{\mathfrak{c}}$ (see (4.7)), $<_{\mathfrak{c}}=<_{\mathfrak{c}}^{\triangleright}$. Similarly, $<_{\mathfrak{c}}^{\triangleleft}=<_{\mathfrak{c}}$.

(ii) Let $C$ be a complete continuous chain from $a$ to $b$. If $C$ is not maximal, there is $u \in Q, u \notin C$, such that either $z<u$ or $u<z$ for each $z \in C$. Let $c=\vee\{z \in C: z<u\}$ and $d=\wedge\{z \in C: u<z\}$. As $C$ is complete, $c, d \in C$ and $c<u<d$. As $C$ is continuous, there is $x \in C$ such that $c<x<d$. Hence either $x<u$, or $u<x$, a contradiction. Thus $C$ is maximal.

For $a \in Q$, we defined in (5.2) the maps $\curlyvee_{a}(x)=a \vee x$ and $\curlywedge_{a}(x)=a \wedge x$ for $x \in Q$. Then

$$
\curlyvee_{a}(x) \leq \curlyvee_{a}(y) \text { and } \curlywedge_{a}(x) \leq \curlywedge_{a}(y) \text { if } x \leq y \text {. }
$$

For each $G \subseteq Q$, its images $\curlyvee_{a}(G)$ and $\curlywedge_{a}(G)$ satisfy (5.3).

A complete lattice $(Q, \leq)$ is modular if

$$
\curlyvee_{a} \curlywedge_{b}=\curlywedge_{b} \curlyvee_{a} \text { for all } a \leq b \text { in } Q .
$$

This is equivalent to the condition that $Q$ has no sublattice $P$ order-isomorphic to a pentagon:

$$
P=\{a, b, c, d, e\}, a<b<c<d, a<e<d, b \vee e=d \text { and } c \wedge e=a .
$$

The lattice $Q$ in Example 6.3(i) is not modular, as it contains pentagons $\left\{\mathbf{0}, a, t, t^{\prime}, \mathbf{1}\right\}$ for $\mathbf{0}<t<$ $t^{\prime}<1$.

Proposition 6.6 Let $C$ be a complete chain from $a$ to $b$ in $Q$ and $z \in Q$.

(i) $\curlywedge_{z}(C)$ is a $\wedge$-complete chain and $\curlyvee_{z}(C)$ is a $\vee$-complete chain.

(ii) Let $Q$ be a modular complete lattice.

1) If $[x, y]$ is a gap then a) either $\curlywedge_{z}(x)=\curlywedge_{z}(y)$, or $\left[\curlywedge_{z}(x), \curlywedge_{z}(y)\right]$ is a gap;

b) either $\curlyvee_{z}(x)=\curlyvee_{z}(y)$, or $\left[\curlyvee_{z}(x), \curlyvee_{z}(y)\right]$ is a gap.

2) If $C$ is continuous then the chains $\curlywedge_{z}(C)$ and $\curlyvee_{z}(C)$ are continuous.

Proof. (i) By (6.1), $\curlywedge_{z}(C)$ is a chain from $z \wedge a$ to $z \wedge b$. Let $\Gamma=\curlywedge_{z}(G)$ for some $G \subseteq C$. As $\wedge G \in C$, we have $\wedge \curlywedge_{z}(G)=\curlywedge_{z}(\wedge G) \in \curlywedge_{z}(C)$ by (5.3). Thus $\curlywedge_{z}(C)$ is $\wedge$-complete. Similarly, $\curlyvee_{z}(C)$ is $\vee$-complete.

(ii) 1) a) Let $[x, y]$ be a gap and $\curlywedge_{z}(x) \neq \curlywedge_{z}(y)$. Assume that $\curlywedge_{z}(x)<u<\curlywedge_{z}(y)$ for some $u \in Q$. Then $u<z$ and $u<y$. If $u \leq x$, then $u \leq \curlywedge_{z}(x)$, a contradiction. Thus $x<u \vee x \leq y$. As $[x, y]$ is a gap, $y=u \vee x$. As $u<z$, it follows from (6.2) that

$$
\curlywedge_{z}(y)=\curlywedge_{z} \curlyvee_{u}(x)=\curlyvee_{u} \curlywedge_{z}(x)=u \curlywedge(x)=u<\curlywedge_{z}(y),
$$

a contradiction. Thus $\left[\curlywedge_{z}(x), \curlywedge_{z}(y)\right]$ is a gap. Similarly, we can prove $b$ ). 
2) Let $C$ be continuous. If $\curlywedge_{z}(C)$ is not continuous, $\left[\curlywedge_{z}(x), \curlywedge_{z}(y)\right]_{\curlywedge_{z}(C)}$ is a gap for some $x<y$ in $C$. Then, for all $\xi \in[x, y]_{C}$, either $\curlywedge_{z}(\xi)=\curlywedge_{z}(x)$, or $\curlywedge_{z}(\xi)=\curlywedge_{z}(y)$. Set $K=\left\{\xi \in[x, y]_{C}\right.$ : $\left.\curlywedge_{z}(\xi)=\curlywedge_{z}(x)\right\}, u=\vee K, L=\left\{\xi \in[x, y]_{C}: \curlywedge_{z}(\xi)=\curlywedge_{z}(y)\right\}$ and $v=\wedge L$. As $C$ is complete, $u, v \in[x, y]_{C}$. As $C$ is continuous, $u=v$.

Let $u \in K$, so that $\curlywedge_{z}(u)=\curlywedge_{z}(x)$ and $u<\xi$ for all $\xi \in L$. As $\curlywedge_{z}(\xi)=\curlywedge_{\xi}(z)$,

$$
\curlyvee_{u} \curlywedge_{z}(y)=\curlyvee_{u} \curlywedge_{z}(\xi)=\curlyvee_{u} \curlywedge_{\xi}(z) \stackrel{(6.2)}{=} \curlywedge_{\xi} \curlyvee_{u}(z)=\xi \wedge \curlyvee_{u}(z) \leq \xi
$$

Thus $\curlyvee_{u} \curlywedge_{z}(y)$ is a lower bound of $L$. Hence $u \vee \curlywedge_{z}(y)=\curlyvee_{u} \curlywedge_{z}(y) \leq u$. So $\curlywedge_{z}(y) \leq u$. As $\curlywedge_{z}(y) \leq z$, we have $\curlywedge_{z}(y) \leq z \wedge u=\curlywedge_{z}(u)=\curlywedge_{z}(x)$, a contradiction. Similarly, we get a contradiction, if we assume that $u \in L$. Thus $\curlywedge_{z}(C)$ is continuous. Similarly, $\curlyvee_{z}(C)$ is continuous.

Corollary 6.7 If $Q$ is modular then $<_{\mathfrak{g}}$ is an $\mathbf{H H}$-relation.

Proof. Let $Q$ by be modular. If $x<_{\mathfrak{g}} y, x \neq y$, then $[x, y]$ is a gap. By Proposition 6.6(ii), for each $z \in Q$, either $x \wedge z=y \wedge z$, or $[x \wedge z, y \wedge z]$ is a gap. So $x \wedge z<_{\mathfrak{g}} y \wedge z$.

Similarly, $x \vee z<_{\mathfrak{g}} y \vee z$. By Definition $3.2,<_{\mathfrak{g}}$ is an HH-relation.

For each $G \subseteq Q$ and any $z \in Q$,

$$
\vee\{z \wedge x: x \in G\} \leq z \wedge(\vee G) \text { and } z \vee(\wedge G) \leq \wedge\{z \vee x: x \in G\}
$$

Consider the Join and Meet Infinite Distributive Identities (JID) and (MID)

$$
z \vee(\wedge G)=\wedge\{z \vee x: x \in G\} \text { and } z \wedge(\vee G)=\vee\{z \wedge x: x \in G\} \text { for all } z \in Q \text { and } G \subseteq Q
$$

For example, the lattice of all subsets of a set $X$ with $\leq=\subseteq, \wedge=\cap$ and $\vee=\cup$ has properties (6.5), while the lattice of all closed subsets of a topological space $X$ does not always have them.

If $Q$ is a chain then conditions (6.5) hold. Note that a lattice $Q$ satisfies (6.5) if and only if $Q$ has a complete embedding into a complete boolean lattice (see [G, Theorem 166]).

Definition 6.8 A lattice $Q$ has properties (JIDC) and (MIDC) if, respectively, the first and second part of (6.5) holds for each chain $G$ in $Q$.

The properties (JIDC) and (MIDC) are weaker than (JID) and (MID). In particular, they do not imply that $Q$ is distributive.

Lemma 6.9 Let $Q$ have properties (JIDC) and (MIDC). If $\ll$ is an $\mathbf{H H}$-relation then

(i) $\ll^{\triangleleft}$ is an $\mathbf{H}$-order and a dual $\mathbf{R}$-order; (ii) $\ll^{\triangleright}$ is an $\mathbf{R}$-order and a dual $\mathbf{H}$-order.

Proof. (i) By Proposition 5.5, $\ll^{\triangleleft}$ is a dual $\mathbf{R}$-order. Let us prove that it is an $\mathbf{H}$-order.

Let $z \in Q$. If $x \ll^{\triangleleft} y$ then there is a complete lower $\ll$-gap chain $T$ from $y$ to $x$ : each $t \in T \backslash\{x\}$ has the immediate «-predecessor $t_{p}$ in $T:\left[t_{p}, t\right]_{T}$ is a gap. Fix $t \in T \backslash\{x\}$ and set $S_{t}=\{s \in T$ : $z \vee s=z \vee t\}$. As $T$ is complete, $u^{t}:=\wedge S_{t} \in T$. By (6.5),

$$
z \vee u^{t}=z \vee\left(\wedge S_{t}\right)=\wedge\left\{z \vee s: s \in S_{t}\right\}=z \vee t \text {, so that } u^{t} \in S_{t}
$$

Let $u^{t} \neq x$. Then $z \vee x \neq z \vee u^{t}=z \vee t$, otherwise $u^{t}=x$ by (6.6). The element $u^{t}$ has the immediate $\ll$-predecessor $u_{p}^{t}$ in $T$, i.e., $u_{p}^{t} \ll u^{t}$. So $z \vee u_{p}^{t} \ll z \vee u^{t}=z \vee t$, as $\ll$ is an H-relation. 
As $u_{p}^{t} \notin S_{t}$, we have $z \vee u_{p}^{t} \neq z \vee t$. So $z \vee u_{p}^{t}$ is the immediate «-predecessor of $z \vee t=z \vee u^{t}$ in $\curlyvee_{z}(T)$. Thus $\curlyvee_{z}(T)$ is a lower «-gap chain from $z \vee y$ to $z \vee x$.

By Proposition 6.6, $\curlyvee_{z}(T)$ is a $\vee$-complete. Hence, by Lemma 4.5(iii), it is complete. Thus $z \vee x \ll^{\triangleleft} z \vee y$, so that $\ll^{\triangleleft}$ is an $\mathbf{H}$-order. The proof of part (ii) is similar.

Theorem 6.10 If a modular lattice $Q$ has properties (JIDC) and (MIDC) then $<_{\mathfrak{c}}$ is an $\mathbf{R R}$-order and $Q$ is a union of disjoint sets each of which has no gaps.

Proof. Let $x<_{\mathfrak{c}} y$ and $C$ be a complete continuous chain from $x$ to $y$. Let $z \in Q$. By Proposition 6.6, $\curlywedge_{z}(C)$ is a $\wedge$-complete, continuous chain from $z \wedge x$ to $z \wedge y$. Let $\Gamma=\curlywedge_{z}(G) \subseteq \curlywedge_{z}(C)$ for some $G \subseteq C$. Then $\vee G \in C$, as $C$ is complete and, by (6.5),

$$
\vee \Gamma=\vee \curlywedge_{z}(G)=\vee\{z \wedge g: g \in G\}=z \wedge(\vee G) \in \curlywedge_{z}(C) .
$$

So $\curlywedge_{z}(C)$ is $\vee$-complete. Thus $\curlywedge_{z}(C)$ is complete, so that $z \wedge x<_{\mathfrak{c}} z \wedge y$. Hence $\leq_{\mathfrak{c}}$ is a dual H-order. Similarly, $<_{\mathfrak{c}}$ is an H-order. By Proposition 6.5, $<_{\mathfrak{c}}=<_{\mathfrak{c}}^{\triangleleft}=<_{\mathfrak{c}}^{\triangleright}$. Thus, by Lemma 6.9, $\leq_{\mathfrak{c}}$ is an RR-order.

By Theorem 2.12, $Q=\cup_{\lambda \in Q_{\mathfrak{r}}}[\mathfrak{p}(\lambda), \lambda]$, all $[\mathfrak{p}(\lambda), \lambda], \lambda \in Q_{\mathfrak{r}}$, are mutually disjoint and $<_{\mathfrak{c}}=\leq$ in each $[\mathfrak{p}(\lambda), \lambda]$. By Proposition 6.2, $[\mathfrak{p}(\lambda), \lambda]$ has no gaps.

As complete chains are modular lattices and have properties (JID) and (MID), we have

Corollary 6.11 Let $Q$ be a complete chain. Then $<_{\mathfrak{g}}$ is an $\mathbf{H H}$-relation, $<_{\mathfrak{g}}^{\triangleleft}$ is an $\mathbf{H}$-order and a dual $\mathbf{R}$-order, $<_{\mathfrak{g}}$ is an $\mathbf{R}$-order and a dual $\mathbf{H}$-order. The relation $<_{\mathfrak{c}}$ is an $\mathbf{R} \mathbf{R}$-order.

\section{Maps and relations in lattices}

In this section we study special subsets in complete lattices - enveloping and inscribing sets. In particular, we consider the lattices $\operatorname{Ref}(Q)$ of relations and $\operatorname{Map}(Q)$ of all maps on a complete lattice $Q$. We describe some of their enveloping and inscribing sets and construct the corresponding radical maps.

\subsection{Enveloping and inscribing sets in lattices, radical maps and topology}

Recall that a subset $L$ is a sublattice of $(Q, \leq)$ if $x \vee y, x \wedge y \in L$ for all $x, y \in L$. It is $\wedge$-complete, if $\wedge G \in L$; and $\vee$-complete, if $\vee G \in L$ for all $G \subseteq L$. It is complete if it is $\wedge$ - and $\vee$-complete.

On the other hand, $L$ can be a lattice in its own right with respect to the restriction of $\leq$ to $L$, not being a sublattice of $Q$. For example, the smallest element in $L$ majorizing $x, y \in L$ may differ from $x \vee y$. Denote it $x \vee^{L} y$. Similarly, we use the notation $x \wedge^{L} y$. A lattice $L$ is $\vee^{L}$-complete if each $G \subseteq L$ has the smallest element $\vee^{L} G$ in $L$ majorizing all $x \in G$. In general, $\vee G \leq \vee^{L} G$. Similarly, if $L$ is $\wedge^{L}$-complete then $\wedge^{L} G \leq \wedge G$. If $L$ is $\vee^{L}$ - and $\wedge^{L}$-complete, it is a complete lattice.

If $L$ is a sublattice of $Q$ then $x \vee y=x \vee^{L} y$ and $x \wedge y=x \wedge^{L} y$ for $x, y \in L$. It is $\wedge$-complete $\left(\vee\right.$-complete), if and only if $\wedge{ }^{L} G=\wedge G\left(\vee^{L} G=\vee G\right)$ for all $G \subseteq L$.

For $a \in Q$ and $G \subseteq Q$, write $G \leq a(a \leq G)$, if $a$ majorizes (minorizes) all $x \in G$. Set

$$
L^{G}=\{a \in L: G \leq a\} \text { and } L_{G}=\{a \in L: a \leq G\} .
$$


Lemma 7.1 (i) Let $L$ be $\wedge^{L}$-complete. If $L$ has the largest element, it is also $\vee^{L}$-complete, i.e., $L$ is a complete lattice; and $\vee^{L} G=\wedge^{L} L^{G}$ is the smallest in $L^{G}$ for all $G \subseteq L$.

(ii) Let $L$ be $\wedge$-complete and $\mathbf{1} \in L$. For $G \subseteq Q, \wedge^{L} L^{G}=\wedge L^{G}$ is the smallest element in $L^{G}$.

(iii) Let $L \subseteq Q$ be $\vee^{L}$-complete. If $L$ has the smallest element, then $L$ is also $\wedge^{L}$-complete, i.e., $L$ is a complete lattice, and $\wedge^{L} G=\vee^{L} L_{G}$ is the largest in $L_{G}$ for all $G \subseteq L$.

(iv) Let $L$ be $\vee$-complete and $\mathbf{0} \in L$. For $G \subseteq Q, \vee^{L} L_{G}=\vee L_{G}$ is the largest element in $L_{G}$.

Proof. Part (i) is proved in Lemma 34 [G]. The proof of (iii) is similar.

(ii) The set $L^{G}$ contains 1. As $L$ is $\wedge$-complete, it follows that $h:=\wedge^{L} L^{G}=\wedge L^{G} \in L$ is the largest element in $Q$ minorizing $L^{G}$. As $g \leq x$ for all $g \in G$ and $x \in L^{G}$, we have $g \leq h$. Thus $h \in L^{G}$ and is the smallest element in $L^{G}$. The proof of part (iv) is similar.

Definition 7.2 (cf. Definition $27[\mathrm{G}]$ ) A set L in $Q$ is enveloping (Cld in [G]) if, for each $x \in Q$, the set $\{l \in L: x \leq l\}$ has the smallest element $\bar{x}$, so that $x \leq \bar{x}$.

It is inscribing if, for each $x \in Q$, the set $\{l \in L: l \leq x\}$ has the largest element $\underline{x}$.

The following theorem refines Corollary $29[\mathrm{G}]$ and characterizes enveloping and inscribing sets in terms of lattice operations.

Theorem 7.3 (i) For $L \subseteq Q$, the following conditions are equivalent.

1) $L$ is enveloping;

2) $L$ is $\wedge$-complete and $\mathbf{1} \in L$;

3) $L$ is a complete lattice, $\wedge$-complete and $\mathbf{1} \in L$;

4) For each $G \subseteq Q$, the set $L^{G}$ (see (7.1)) has the smallest element $m_{G}$ and

$$
m_{G}=\wedge^{L} L^{G}=\wedge L^{G}=\vee^{L}\{\bar{x}: x \in G\} ; \quad \text { if } G \subseteq L \text { then } m_{G}=\vee^{L} G .
$$

(ii) For $N \subseteq Q$, the following conditions are equivalent.

1) $N$ is inscribing;

2) $N$ is $\vee$-complete and $\mathbf{0} \in N$;

3) $N$ is a complete lattice, $\vee$-complete and $\mathbf{0} \in N$;

4) For each $G \subseteq Q$, the set $N_{G}$ (see (7.1)) has the largest element $n_{G}$ and

$$
n_{G}=\vee^{N} N_{G}=\vee N_{G}=\wedge^{N}\{\underline{x}: x \in G\} ; \quad \text { if } G \subseteq N \text { then } n_{G}=\wedge^{N} G .
$$

Proof. (i) 1$) \Rightarrow 2$ ) As $L$ is enveloping, $\overline{\mathbf{1}}=\mathbf{1} \in L$. For $G \subseteq L$, let $t=\wedge G$. As $t \leq g$ for all $g \in G$, and $\bar{t}$ is the smallest element in $L$ majorizing $t, t \leq \bar{t} \leq g$. So $t \leq \bar{t} \leq \wedge G=t$. Thus $t=\bar{t} \in L$ and $L$ is $\wedge$-complete.

2) $\Rightarrow 3$ ) If $L$ is $\wedge$-complete and $\mathbf{1} \in L$, it is a complete lattice by Lemma 7.1(i).

$3) \Rightarrow 4)$ Let $G \subseteq Q$. By Lemma 7.1(ii), $\wedge \wedge^{L} L^{G}$ exists, $\wedge{ }^{L} L^{G}=\wedge L^{G}$ and it is the smallest element in $L^{G}$. So $m_{G}=\wedge^{L} L^{G}=\wedge L^{G}$. If $G \subseteq L$ then $m_{G}=\vee^{L} G$ by Lemma 7.1(i).

For each $x \in G$, the set $\{l \in L: x \leq l\}$ majorizing $x$ has the smallest element $\bar{x}$. As $L$ is a complete lattice, it has the smallest element $s=\vee^{L}\{\bar{x}: x \in G\}$ majorizing all $\bar{x}$. Thus $x \leq \bar{x} \leq s$ for all $x \in G$. So $s \in L^{G}$. 
If $y \in L$ majorizes all $x \in G$, then $\bar{x} \leq y$ for all $x \in G$ (by definition of $\bar{x}$ ), i.e., $y$ majorizes all $\bar{x}$. Therefore $s \leq y$. Hence $s$ is the smallest element in $L$ majorizing all $x \in G$, i.e., $s$ is the smallest element in $L^{G}$. Thus $s=m_{G}=\wedge^{L} L^{G}=\wedge L^{G}=\vee^{L}\{\bar{x}: x \in G\}$.

$4) \Rightarrow 1$ ) For each $x \in Q$, take $G=\{x\}$.

The proof of (ii) is similar.

Let $\left\{L_{\lambda}\right\}_{\lambda \in \Lambda}$ be subsets of $Q$. Consider the set $L_{\Lambda}=\left\{x=\left\{x_{\lambda}\right\}_{\lambda \in \Lambda}: x_{\lambda} \in L_{\lambda}\right\}$ and let

$$
\widehat{L_{\Lambda}}=\left\{\wedge x:=\wedge_{\lambda \in \Lambda} x_{\lambda} \text { for } x \in L_{\Lambda}\right\} \text { and } L_{\Lambda}^{\vee}=\left\{\vee x:=\vee_{\lambda \in \Lambda} x_{\lambda} \text { for } x \in L_{\Lambda}\right\} .
$$

Corollary 7.4 Let all $\left\{L_{\lambda}\right\}_{\lambda \in \Lambda}$ be enveloping and $\left\{N_{\lambda}\right\}_{\lambda \in \Lambda}$ inscribing sets in $Q$. Then

(i) The sets $\cap_{\lambda \in \Lambda} L_{\lambda}, \widehat{L_{\Lambda}}$ and $\cup_{\lambda \in \Lambda} L_{\lambda}$ are enveloping in $Q$.

(ii) The sets $\cup_{\lambda \in \Lambda} N_{\lambda}, N_{\Lambda}$ and $\cap_{\lambda \in \Lambda} N_{\lambda}$ are inscribing in $Q$.

Proof. (i) All $L_{\lambda}$ are $\wedge$-complete by Theorem 7.3. So $\cap_{\lambda \in \Lambda} L_{\lambda}$ is $\wedge$-complete. As $\mathbf{1} \in L_{\lambda}$ for all $\lambda \in \Lambda, \mathbf{1} \in \cap_{\lambda \in \Lambda} L_{\lambda}$. By Theorem 7.3, $\cap_{\lambda \in \Lambda} L_{\lambda}$ is enveloping.

Let $g \in G \subseteq \hat{L_{\Lambda}}$. By (7.3), $g=\wedge_{\lambda \in \Lambda} g_{\lambda}$ for some $\left\{g_{\lambda}\right\}_{\lambda \in \Lambda} \in L_{\Lambda}$, and

$$
\wedge G=\wedge_{g \in G} g=\wedge_{g \in G}\left(\wedge_{\lambda \in \Lambda} g_{\lambda}\right) .
$$

For $\lambda \in \Lambda$, set $y_{\lambda}=\wedge_{g \in G} g_{\lambda}$. As $g \leq g_{\lambda}$, we have $\wedge_{g \in G} g \leq \wedge_{g \in G} g_{\lambda}=y_{\lambda}$. So $\wedge G \leq \wedge_{\lambda \in \Lambda} y_{\lambda}$. On the other hand, as $y_{\lambda} \leq g_{\lambda}$ for each $g \in G$, we have $\wedge_{\lambda \in \Lambda} y_{\lambda} \leq \wedge_{\lambda \in \Lambda} g_{\lambda}=g$. Thus

$$
\wedge G \leq \wedge_{\lambda \in \Lambda} y_{\lambda} \leq \wedge_{g \in G} g=\wedge G, \text { so that } \wedge G=\wedge_{\lambda \in \Lambda} y_{\lambda}
$$

As all $L_{\lambda}$ are $\wedge$-complete by Theorem 7.3, all $y_{\lambda} \in L_{\lambda}$. So $\left\{y_{\lambda}\right\}_{\lambda \in \Lambda} \in L_{\Lambda}$ and $\wedge_{\lambda \in \Lambda} y_{\lambda} \in \widehat{L_{\Lambda}}$. Hence $\wedge G \in \widehat{L_{\Lambda}}$. Thus $\widehat{L_{\Lambda}}$ is $\wedge$-complete and $\mathbf{1} \in \widehat{L_{\Lambda}}$ as $\mathbf{1} \in L_{\lambda}$ for all $\lambda$. By Theorem $7.3, \widehat{L_{\Lambda}}$ is enveloping.

Now let $G \subseteq \cup_{\lambda \in \Lambda} L_{\lambda}$. Set $G_{\lambda}=G \cap L_{\lambda}$ for $\lambda \in \Lambda$. Then $\wedge G_{\lambda} \in L_{\lambda}$ for all $\lambda \in \Lambda$. Hence

$$
\left\{\wedge G_{\lambda}\right\}_{\lambda \in \Lambda} \in L_{\Lambda} \text { and } \wedge G=\wedge\left(\cup_{\lambda \in \Lambda} G_{\lambda}\right) \stackrel{(5.4)}{=} \wedge_{\lambda \in \Lambda}\left(\wedge G_{\lambda}\right) \stackrel{(7.3)}{\in} \hat{L_{\Lambda}}
$$

So $\cup_{\lambda \in \Lambda} L_{\lambda}$ is $\wedge$-complete. As it contains $\mathbf{1}$, it is enveloping by Theorem 7.3.

Part (ii) can be proved similarly.

A map $g: Q \rightarrow Q$ is called

a T-radical map if $x \leq g(x)=g(g(x))$ and $g(x \vee y)=g(x) \vee g(y)$,

a dual T-radical map if $g(g(x))=g(x) \leq x$ and $g(x \wedge y)=g(x) \wedge g(y)$ for $x, y \in Q$.

$T$-radical maps are radical (see (2.13)). Indeed, if $x \leq y$ then $g(x) \leq g(x) \vee g(y)=g(x \vee y)=g(y)$. Similarly, dual $T$-radical maps are dual radical maps.

For a map $g: Q \rightarrow Q$, we denote by $\operatorname{Fix}(g)$ the set of all $g$-fixed points:

$$
\operatorname{Fix}(g)=\{x \in Q: g(x)=x\} .
$$

For an enveloping set $L$ and an inscribing set $N$, define the maps $f_{L}$ and $g_{N}$ on $Q$ by

$$
f_{L}: x \in Q \mapsto \bar{x} \in L, \text { and } g_{N}: x \in Q \mapsto \underline{x} \in N
$$


Proposition 7.5 (i) $\theta: L \mapsto f_{L}$ is a bijection from the class of all enveloping sets in $Q$ onto the class of all radical maps on $Q$. Its inverse $\theta^{-1}: g \mapsto \operatorname{Fix}(g)$. Moreover, $L=\operatorname{Fix}\left(f_{L}\right)$ and $g=f_{\operatorname{Fix}(g)}$.

(ii) $\theta$ is a bijection from the class of all enveloping sublattices of $Q$ onto the class of all T-radical maps on $Q$.

(iii) $\phi: N \mapsto g_{N}$ is a bijection from the class of all inscribing sets in $Q$ onto the class of all dual radical maps on $Q$. Its inverse $\phi^{-1}: f \mapsto \operatorname{Fix}(f)$. Moreover, $N=\operatorname{Fix}\left(g_{N}\right)$ and $f=g_{\operatorname{Fix}(f)}$.

(iv) $\phi$ is a bijection from the class of all inscribing sublattices of $Q$ onto the class of all dual $T$-radical maps on $Q$.

Proof. Part (i) was proved in [G, Lemma 28]. The proof of (iii) is similar.

(ii) Let $L$ be an enveloping sublattice of $Q$. Set $f=f_{L}$. By (i), $f$ is a radical map. Hence, for $x, y \in Q, x \leq f(x) \leq f(x \vee y)$ and $y \leq f(y) \leq f(x \vee y)$. So $x \vee y \leq f(x) \vee f(y) \leq f(x \vee y)$. Thus $f(x \vee y) \leq f(f(x) \vee f(y)) \leq f(f(x \vee y))=f(x \vee y)$, so that $f(x \vee y)=f(f(x) \vee f(y))$. As $L$ is a sublattice, $f(x) \vee f(y) \in L=\operatorname{Fix}(f)$. Hence $f(x \vee y)=f(x) \vee f(y)$. Thus $f$ is a $T$-radical map.

Conversely, let $g$ be a $T$-radical map. By (i), $\operatorname{Fix}(g)$ is an enveloping set. By Theorem 7.3, $\operatorname{Fix}(g)$ is $\wedge$-complete. If $x, y \in \operatorname{Fix}(g)$ then $g(x \vee y)=g(x) \vee g(y)=x \vee y$. So $\operatorname{Fix}(g)$ is an enveloping sublattice of $Q$ which completes the proof of (ii). Part (iv) can be proved similarly.

Consider the following link between $T$-radical maps and topology. For a set $X$, the set $\mathrm{P}(X)$ of all subsets of $X$ is a complete lattice with order $\leq=\subseteq, \mathbf{0}=\varnothing, \mathbf{1}=X, \wedge=\cap$ and $\vee=\cup$.

For a topological space $(X, \tau)$, denote by $\tau^{\text {op }}$ the $\vee$-complete sublattice of all open subsets in $\mathrm{P}(X)$ and by $\tau^{\mathrm{cl}}$ the $\wedge$-complete sublattice of all closed subsets in $\mathrm{P}(X)$.

Proposition 7.6 (i) Let $(X, \tau)$ be a topological space and $Q=\mathrm{P}(X)$.

1) If $f: Q \rightarrow Q$ maps each $G \in Q$ into its $\tau$-closure then $f$ is a T-radical map.

2) If $g: Q \rightarrow Q$ maps each $G \in Q$ into its $\tau$-interior then $g$ is a dual T-radical map.

(ii) Let $X$ be a set and $Q=\mathrm{P}(X)$. Let $f: Q \rightarrow Q$ be a map.

1) If $f$ is a T-radical map and $f(\varnothing)=\varnothing$, then $(X, \tau)$ with $\tau^{\mathrm{cl}}=\{f(G): G \in Q\}$ is a topological space.

2) If $f$ is a dual T-radical map and $f(X)=X$, then $(X, \tau)$ with $\tau^{\text {op }}=\{f(G): G \in Q\}$ is a topological space.

Proof. (i) 1) For $G \in Q$, its $\tau$-closure $f(G)=\cap\left\{F \in \tau^{\mathrm{cl}}: G \subseteq F\right\} \in \tau^{\mathrm{cl}}$. Hence $G \subseteq$ $f(G)=f(f(G))$. Let $K \in Q$. As $\tau^{\mathrm{cl}}$ is a sublattice of $Q, G \cup K \subseteq f(G) \cup f(K) \in \tau^{\mathrm{cl}}$. Hence $f(G \cup K) \subseteq f(G) \cup f(K) \subseteq f(G \cup K)$. Thus $f(G \cup K)=f(G) \cup f(K)$, so that $f$ is a $T$-radical map. Part 2) follows from duality.

(ii) 1) Let $L=\operatorname{Fix}(f)=\{G \in Q: f(G)=G\}$. Then $L=\{f(G): G \in Q\}$. By Proposition 7.5, $L$ is an enveloping sublattice. By Theorem 7.3, it is $\cap$-complete. By (7.4), $f(G) \cup f(K)=$ $f(G \cup K) \in L$ for $G, K \in Q$. Hence $L$ is a $\cap$-complete sublattice of $Q$. As $X \subseteq f(X) \subseteq X$ by (7.4), $X=f(X) \in L$. As $f(\varnothing)=\varnothing, \varnothing \in L$. Then $L=\tau^{\text {cl }}$ for some topology $\tau$ in $X$. Part 2) follows from duality. 


\subsection{Enveloping and inscribing sets in $\operatorname{Ref}(Q)$}

For a complete lattice $(Q, \leq), \operatorname{Ref}(Q)$ is also a complete lattice with the order $\subseteq$ (see (1.1)). For a subset $F \subseteq \operatorname{Ref}(Q)$, the relations $\wedge F=\ll_{\wedge F}:=\cap_{\ll \in F} \ll$ and $\vee F=\ll_{\vee F}:=\cup_{\ll \in F} \ll$ are defined in (5.6). Consider the following subsets of $\operatorname{Ref}(Q)$ :

$$
\begin{gathered}
L_{\mathbf{H}}=\{\text { all } \mathbf{H} \text {-relations in } \operatorname{Ref}(Q)\}, L_{d \mathbf{H}}=\{\text { all dual H-relations in } \operatorname{Ref}(Q)\}, \\
L_{\mathrm{uc}}=\{\text { all up-contiguous } \ll \operatorname{in} \operatorname{Ref}(Q)\}, L_{\mathrm{dc}}=\{\text { all down-contiguous } \ll \text { in } \operatorname{Ref}(Q)\}, \\
L_{\mathrm{ue}}=\{\text { all up-expanded } \ll \operatorname{in} \operatorname{Ref}(Q)\}, L_{\mathrm{de}}=\{\text { all down-expanded } \ll \text { in } \operatorname{Ref}(Q)\}, \\
L_{\triangleright}=\left\{\ll i n \operatorname{Ref}(Q): \ll=\ll^{\triangleright}\right\}, L_{\triangleleft}=\left\{\ll \operatorname{in} \operatorname{Ref}(Q): \ll=\ll^{\triangleleft}\right\} .
\end{gathered}
$$

Theorem 7.7 (i) The sets $L_{\mathbf{H}}, L_{d \mathbf{H}}, L_{\mathrm{uc}}, L_{\mathrm{dc}}$ are inscribing and enveloping sublattices of $\operatorname{Ref}(Q)$.

(ii) The sets $L_{\triangleright}, L_{\triangleleft}, L_{\mathrm{ue}}, L_{\mathrm{de}}$ and the set $L_{\mathrm{o}}$ of all orders are enveloping in $\operatorname{Ref}(Q)$.

Proof. (i) Let $F \subseteq L_{\mathbf{H}}$. If $a \ll_{\wedge F} b$ (see (5.6)) then $a \ll b$ for all $\ll$ in $F$. As all $\ll$ are H-relations, $a \vee x \ll b \vee x$ for all $x \in Q$. Hence $a \vee x \ll_{\wedge F} b \vee x$. So $\wedge F$ is an H-relation. Let now $a \ll_{\vee F} b$ (see (5.6)). Then $a \ll b$ for some $\ll$ in $F$, and $a \vee x \ll b \vee x$ for all $x \in Q$. Hence $a \vee x \ll_{\vee F} b \vee x$. So $\vee F$ is an $\mathbf{H}$-relation. Thus $L_{\mathbf{H}}$ is complete. As $\mathbf{0 , 1} \in L_{\mathbf{H}}, L_{\mathbf{H}}$ is an inscribing and enveloping lattice by Theorem 7.3. The proof for $L_{d \mathbf{H}}$ is similar.

Let $F \subseteq L_{\mathrm{uc}}$. If $a \ll_{\wedge F} b$ then $a \ll b$ for all $\ll$ in $F$. So

$$
\left[a, \ll_{\wedge F}\right]=\cap_{\ll \in F}[a, \ll] \text { and }\left[\ll_{\wedge F}, b\right]=\cap_{\ll \in F}[\ll, b] .
$$

As all $\ll$ are up-contiguous, $[a, b] \subseteq[\ll, b]$ for all $\ll$ in $F$. By $(7.6),[a, b] \subseteq\left[\ll_{\wedge F}, b\right]$. So $\wedge F$ is up-contiguous. Let now $a \ll_{\vee F} b$. Then $a \ll b$ for some $\ll \operatorname{in} F$. Hence $[a, b] \subseteq[\ll, b]$. Thus $[a, b] \subseteq\left[\ll_{\vee F}, b\right]$. So $\vee F$ is up-contiguous. Thus $L_{\mathrm{uc}}$ is complete. As $\mathbf{1}$ and $\mathbf{0}$ are up-contiguous, $L_{\mathrm{uc}}$ is an inscribing and enveloping lattice by Theorem 7.3. The proof for $L_{\mathrm{dc}}$ is similar.

(ii) For $F \subseteq L_{\triangleright}$, let $a \ll_{\wedge F}^{\triangleright} b$. By (4.6), there is a complete, upper $\ll_{\wedge F}$-gap chain $C$ from $a$ to $b$, i.e., each $x \in C \backslash\{b\}$ has an immediate $\ll_{\wedge F}$-successor $s_{x}: x \ll_{\wedge F} s_{x}$ and $\left[x, s_{x}\right] \cap C=\left\{x, s_{x}\right\}$. By (5.6), $x \ll s_{x}$ for all $\ll$ in $F$. So $s_{x}$ is an immediate $\ll$-successor of $x$ in $C$. Thus $C$ is a complete, upper $\ll$-gap chain for all $\ll$ in $F$, i.e., $a \ll^{\triangleright} b$. As $\ll=\ll^{\triangleright}, a \ll b$ for all $\ll$ in $F$. Hence $a \ll_{\wedge F} b$. So $\ll_{\wedge F}^{\triangleright}$ is stronger than $\ll_{\wedge F}$. By (4.7), $\ll_{\wedge F}$ is stronger than $\ll_{\wedge F}^{\triangleright}$. Hence $\ll_{\wedge F}^{\triangleright}=\ll_{\wedge F}$. Thus $L_{\triangleright}$ is $\wedge$-complete. As $\mathbf{1} \in L_{\triangleright}, L_{\triangleright}$ is enveloping by Theorem 7.3. The proof for $L_{\triangleleft}$ is similar.

For $F \subseteq L_{\mathrm{ue}}$ and $a \in Q$, let $G \subseteq\left[a, \ll_{\wedge F}\right]$. By $(7.6), G \subseteq[a, \ll]$ for all $\ll$ in $F$. As all $\ll$ are up-expanded, $\vee G \in[a, \ll]$ for all $\ll$ in $F$. Hence $\vee G \in\left[a, \ll_{\wedge F}\right]$. So $\ll_{\wedge F}$ is up-expanded. Thus $L_{\text {ue }}$ is $\wedge$-complete. As $\mathbf{1} \in L_{\mathrm{ue}}, L_{\mathrm{ue}}$ is enveloping by Theorem 7.3. The proof for $L_{\mathrm{de}}$ is similar.

For $F \subseteq L_{\mathrm{o}}$, let $a \ll_{\wedge F} b \ll_{\wedge F} c$. Then $a \ll b \ll c$ for all $\ll$ in $F$, whence $a \ll c$. So $a \ll_{\wedge_{F}} c$. Thus $\ll_{\wedge F}$ is an order. Thus $L_{\mathrm{O}}$ is $\wedge$-complete. As $\mathbf{1} \in L_{\mathrm{o}}, L_{\mathrm{O}}$ is enveloping by Theorem 7.3.

Corollary 7.8 The sets of all $\mathbf{T}$-orders, of all dual $\mathbf{T}$-orders, of all $\mathbf{R}$-orders, of all dual $\mathbf{R}$-orders are enveloping in $\operatorname{Ref}(Q)$.

Proof. By Definition 2.3, the set of all T-orders is the intersection of $L_{\mathrm{o}}, L_{\mathrm{uc}}, L_{\mathrm{ue}}$ which are enveloping. So it is enveloping by Corollary 7.4. The rest of the proof is similar.

By Proposition 7.5, there is a bijection between enveloping sets $L$ and radical maps $f_{L}$. It is, however, often difficult to determine the action of $f_{L}$. Below we do it for some sets in $\operatorname{Ref}(Q)$. 
Lemma 7.9 The radical maps $f_{L_{\triangleright}}, f_{L_{\triangleleft}}$ on $\operatorname{Ref}(Q)$ act by $f_{L_{\triangleright}}: \ll \mapsto \ll^{\triangleright}$ and $f_{L_{\triangleleft}}$ : $\mapsto \ll^{\triangleleft}$.

Proof. Set $g: \ll \mapsto \ll^{\triangleright}$. Then $g(g(\ll))=\left(\ll^{\triangleright}\right)^{\triangleright} \stackrel{(4.8)}{=} \ll^{\triangleright}=g(\ll) \supseteq \ll$. It is easy to check that $\ll \subseteq \prec$ implies $g(\ll)=\ll^{\triangleright} \subseteq \prec^{\triangleright}=g(\prec)$, as each complete upper «-gap chain is also a complete upper $\prec$-gap chain. Thus $(2.13)$ holds. So $g$ is a radical map from $\operatorname{Ref}(Q)$ onto $L_{\triangleright}$. As $\operatorname{Fix}(g)=L_{\triangleright}$, we have from Proposition 7.5 that $g=f_{\mathrm{Fix}(g)}=f_{L_{\triangleright}}$. The proof for $f_{L_{\triangleleft}}$ is similar.

As $L_{\mathbf{H}}$ and $L_{d \mathbf{H}}$ are inscribing and enveloping sublattices of $\operatorname{Ref}(Q)$, the maps $f_{L_{\mathbf{H}}}$ and $f_{L_{d \mathbf{H}}}$ are $T$-radical, and $g_{L_{\mathbf{H}}}$ and $g_{L_{d \mathbf{H}}}($ see $(7.5))$ are dual $T$-radical. We describe their action below.

Proposition 7.10 (i) Set $\prec_{1}=g_{L_{\mathbf{H}}}(\ll)$. Then $a \prec_{1} b$ if $a \vee x \ll b \vee x$ for all $x \in Q$.

(ii) Set $\prec_{2}=g_{L_{d \mathbf{H}}}(\ll)$. Then $a \prec_{2} b$ if $a \wedge x \ll b \wedge x$ for all $x \in Q$.

(iii) Set $\prec_{3}=f_{L_{\mathbf{H}}}(\ll)$. Then $a \prec_{3} b$ if there are $x \ll y$ such that $x \leq a$ and $b=a \vee y$.

(iv) Set $\prec_{4}=f_{L_{d \mathbf{H}}}(\ll)$. Then $a \prec_{4} b$ if there are $x \ll y$ such that $a=b \wedge x$ and $b \leq y$.

Proof. (i) By Lemma 3.1, $\prec_{1}$ is an $\mathbf{H}$-relation. Set $x=\mathbf{0}$. We get that $\prec_{1}$ stronger than $\ll$ :

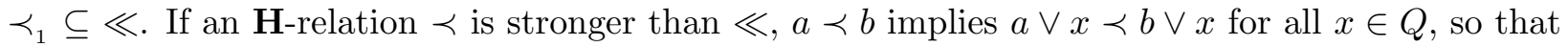
$a \vee x \ll b \vee x$. Thus $a \prec_{1} b$. So $\prec$ is stronger than $\prec_{1}: \prec \subseteq \prec_{1}$. Hence $\prec_{1}$ is the largest H-relation minorizing $\ll$. By Definition 7.2 and $(7.5), g_{L_{\mathbf{H}}}(\ll)=\prec_{1}$. The proof of (ii) is similar.

(iii) Let $a \prec_{3} b$. Then there are

$$
x, y \in Q \text { such that } x \ll y, x \leq a \text { and } b=a \vee y \text {. }
$$

For $z \in Q, x \leq a \vee z$ and $b \vee z=(a \vee y) \vee z=(a \vee z) \vee y$. Hence $(a \vee z) \prec_{3}(b \vee z)$. Thus $\prec_{3}$ is an H-relation by Lemma 3.1. It majorizes $\ll\left(\ll \subseteq \prec_{3}\right)$, as $a \ll b$ implies $a \prec_{3} b$ (set $x=a, y=b$ ).

Let an H-relation $\prec$ majorize $\ll(\ll \subseteq \prec)$. If $a \prec_{3} b$ then (7.7) holds. As $x \ll y$ and $\ll \subseteq \prec$, we have $x \prec y$. As $\prec$ is an H-relation, it follows from (7.7) that $a=(a \vee x) \prec(a \vee y)=b$. Thus $\prec_{3}$ is stronger than $\prec: \prec_{3} \subseteq \prec$. Hence $\prec_{3}$ is the smallest H-relation majorizing $\ll$, i.e., $\prec_{3}=f_{L_{\mathbf{H}}}(\ll)$. The proof of (iv) is similar.

For each $\ll$ in $\operatorname{Ref}(Q)$, define the relations $\ll_{\mathrm{uc}}$ and $\ll_{\mathrm{dc}}$ as follows:

$$
\begin{aligned}
& a \ll_{\mathrm{uc}} b \text { if and only if there is } c \in Q \text { satisfying } c \ll b \text { and } a \in[c, b] \text {; } \\
& a \ll_{\mathrm{dc}} b \text { if and only if there is } c \in Q \text { such that } a \ll c \text { and } b \in[a, c] .
\end{aligned}
$$

Proposition 7.11 For each $\ll$ in $\operatorname{Ref}(Q), \ll_{\mathrm{uc}}=f_{L_{\mathrm{uc}}}(\ll)$ and $\ll_{\mathrm{dc}}=f_{L_{\mathrm{dc}}}(\ll)$.

Proof. If $a \ll_{\mathrm{uc}} b$ then $x \in[c, b]$ for $x \in[a, b]$. Then, by $(7.8), x \ll_{\mathrm{uc}} b$. Hence $[a, b] \subseteq\left[\ll_{\mathrm{uc}}, b\right]$. So (see Definition 2.1) $\ll_{\mathrm{uc}}$ is up-contiguous.

If $a \ll b$ then $a \ll_{\mathrm{uc}} b$ (set $c=a$ in (7.8)). So $\ll_{\mathrm{uc}}$ majorizes $\ll\left(\ll \subseteq \ll_{\mathrm{uc}}\right.$ see $\left.(1.1)\right)$.

Let an up-contiguous relation $\prec$ majorize $\ll(\ll \subseteq \prec)$. If $a \ll$ uc $b$ then $c \ll b$ and $a \in[c, b]$ for some $c \in Q$. Hence $c \prec b$. As $\prec$ is up-contiguous, $[c, b] \subseteq[\prec, b]$, so that $a \prec b$. Thus $\ll_{\text {uc }}$ is stronger than $\prec$. So $\ll_{\mathrm{uc}}$ is the smallest element of $L_{\mathrm{uc}}$ majorizing $\ll$, i.e., $\ll_{\mathrm{uc}}=f_{L_{\mathrm{uc}}}(\ll)$. The equality $f_{L_{\mathrm{dc}}}(\ll)=\ll_{\mathrm{dc}}$ is proved similarly. 


\subsection{Enveloping and inscribing sets of maps on lattices}

For a complete lattice $(Q, \leq)$, denote by $\operatorname{Map}(Q)$ the set of all maps $Q \rightarrow Q$. For $f, g \in \operatorname{Map}(Q)$,

$$
g \lesssim f \text { if } g(x) \leq f(x) \text { for all } x \in Q ; \text { and set } \mathbf{0}_{\mathrm{Map}}: x \mapsto \mathbf{0} \text { and } \mathbf{1}_{\mathrm{Map}}: x \mapsto \mathbf{1} .
$$

Then $(\operatorname{Map}(Q), \lesssim)$ is a complete lattice and, for each $G \subseteq \operatorname{Map}(Q)$,

$$
(\vee G)(x)=\vee_{f \in G} f(x) \text { and }(\wedge G)(x)=\wedge_{f \in G} f(x) .
$$

Let $\operatorname{Rad}_{Q}$ and $\operatorname{dRad}_{Q}$ be the sets of all radical and dual radical maps, respectively, on $Q$ (we write Rad and dRad). They are partially ordered with respect to the order $\lesssim$,

$$
\begin{gathered}
\mathbf{0}_{\mathrm{Rad}}: x \mapsto x \text { and } \mathbf{1}_{\mathrm{Rad}}=\mathbf{1}_{\mathrm{Map}}: x \mapsto \mathbf{1} \text { for all } x \in Q ; \\
\mathbf{0}_{\mathrm{dRad}}=\mathbf{0}_{\mathrm{Map}}: x \mapsto \mathbf{0} \text { and } \mathbf{1}_{\mathrm{dRad}}=\mathbf{0}_{\mathrm{Rad}}: x \mapsto x \text { for all } x \in Q .
\end{gathered}
$$

Lemma 7.12 (i) Let $f$ and $g$ be radical maps in $Q$. The following conditions are equivalent:
1) $g \lesssim f$
2) $f(g(x))=f(x)$ for all $x \in Q$;
3) $\operatorname{Fix}(f) \subseteq \operatorname{Fix}(g)$.

(ii) For $G \subseteq \operatorname{Rad}$, the set $\cap_{g \in G} \operatorname{Fix}(g)$ is enveloping.

Proof. (i) 1) $\Rightarrow 2$ ) Let $x \in Q$. By (2.13), $x \leq g(x) \leq f(x)$, so that $f(x) \leq f(g(x)) \leq$ $f(f(x))=f(x)$. Thus $f(g(x))=f(x)$.

$2) \Rightarrow 3)$ If $x \in \operatorname{Fix}(f)$ then, by $(2.13), x \leq g(x) \leq f(g(x))=f(x)=x$. So $g(x)=x$ and $\operatorname{Fix}(f) \subseteq \operatorname{Fix}(g)$.

$3) \Rightarrow 1)$ For all $x \in Q, f(x) \in \operatorname{Fix}(f) \subseteq \operatorname{Fix}(g)$. So, by (2.13), $g(x) \leq g(f(x))=f(x)$.

(ii) By Proposition 7.5, $\operatorname{Fix}(g)$ are enveloping sets for $g \in G$. So $\cap_{g \in G} \operatorname{Fix}(g)$ is enveloping by Corollary 7.4 .

Theorem 7.13 (i) $\operatorname{Rad}$ is an enveloping set in $\operatorname{Map}(Q)$.

(ii) For $G \subseteq \operatorname{Rad}$, set $\mathfrak{h}=\vee^{\operatorname{Rad}} G$ and $\mathfrak{g}=\wedge G$. Then $\mathfrak{h}, \mathfrak{g} \in \operatorname{Rad}$, Fix $(\mathfrak{h})=\cap_{g \in G} \operatorname{Fix}(g)$,

$$
\operatorname{Fix}(\mathfrak{g})=\left(\cup_{g \in G} \operatorname{Fix}(g)\right) \cup \widehat{K_{G}} \text {, where } K_{G}=\left\{\left\{x_{g}\right\}_{g \in G}: x_{g} \in \operatorname{Fix}(g)\right\}(\text { see }(7.3)) \text {. }
$$

The set $\operatorname{Fix}(\mathfrak{g})$ is the smallest enveloping set in $Q$ containing $\cup_{g \in G} \operatorname{Fix}(g)$.

Proof. (i) For $G \subseteq \operatorname{Rad}$, set $\gamma=\wedge G \in \operatorname{Map}(Q)$. As $x \leq f(x)$ for all $x \in Q, f \in G$ by (2.13), we have $x \leq \gamma(x) \leq f(x)$ by (7.10). From this and (2.13) it follows that for all $x \in Q$,

$$
\gamma(x) \leq \gamma(\gamma(x)) \leq f(\gamma(x)) \leq f(f(x))=f(x) \text { for all } f \in G .
$$

Hence $\gamma(x) \leq \gamma(\gamma(x)) \leq \wedge_{f \in G} f(x)=\gamma(x)$. Thus $x \leq \gamma(x)=\gamma(\gamma(x))$ for all $x \in Q$.

Let $x \leq y$. Then $f(x) \leq f(y)$ for all $f \in G$. Hence $\gamma(x)=\wedge_{f \in G} f(x) \leq \wedge_{f \in G} f(y)=\gamma(y)$. Thus $\gamma \in \operatorname{Rad}$. So $\operatorname{Rad}$ is $\wedge$-complete. As $\mathbf{1}_{\text {Map }} \in \operatorname{Rad}$, Rad is an enveloping set by Theorem 7.3.

(ii) As Rad is enveloping, it is complete and $\wedge$-complete by Theorem 7.3. Thus $\mathfrak{h}, \mathfrak{g} \in \operatorname{Rad}$.

By Lemma 7.12, $L:=\cap_{g \in G} \operatorname{Fix}(g)$ is enveloping. By Proposition 7.5,L=Fix $\left(f_{L}\right)$ for some radical map $f_{L}$. As $\operatorname{Fix}\left(f_{L}\right) \subseteq \operatorname{Fix}(g)$ for all $g \in G, g \lesssim f_{L}$ by Lemma 7.12. As $\mathfrak{h}$ is the smallest radical map majorizing all $g \in G, g \lesssim \mathfrak{h} \lesssim f_{L}$. So, by Lemma $7.12, L=\operatorname{Fix}\left(f_{L}\right) \subseteq \operatorname{Fix}(\mathfrak{h}) \subseteq$ $\cap_{g \in G} \operatorname{Fix}(g)=L$. Thus $\operatorname{Fix}(\mathfrak{h})=L=\cap_{g \in G} \operatorname{Fix}(g)$. 
As $\mathfrak{g} \in \operatorname{Rad}$, it is the largest radical map minorizing all $g \in G$. Hence, by Proposition 7.5 and Lemma 7.12, $\operatorname{Fix}(\mathfrak{g})$ is an enveloping set in $Q$ and $F:=\cup_{g \in G} \operatorname{Fix}(g) \subseteq \operatorname{Fix}(\mathfrak{g})$. Let $S$ be an enveloping set such that $F \subseteq S$. Then $S=\operatorname{Fix}\left(f_{S}\right)$ for a radical map $f_{S}$, and $f_{S} \lesssim g$ for $g \in G$. Hence $f_{S} \lesssim \mathfrak{g}$, so that $\operatorname{Fix}(\mathfrak{g}) \subseteq S$. Thus $\operatorname{Fix}(\mathfrak{g})$ is the smallest enveloping set containing $F$.

Let $R=F \cup \widehat{K_{G}}$ (see (7.3) and (7.11)). By Corollary 7.4, $R$ is enveloping. Hence, as above, $F \subseteq \operatorname{Fix}(\mathfrak{g}) \subseteq R$. For $x=\left\{x_{g}\right\} \in K_{G}, \wedge x=\wedge_{g \in G} x_{g} \in \widehat{K_{G}}$ and $\wedge x \leq x_{g}$ for all $g \in G$. Hence $\mathfrak{g}(\wedge x) \leq \mathfrak{g}\left(x_{g}\right)=x_{g}$, as $\operatorname{Fix}(g) \subseteq \operatorname{Fix}(\mathfrak{g})$. So $\mathfrak{g}(\wedge x) \leq \wedge_{g \in G} x_{g}=\wedge x$. As $\mathfrak{g}$ is radical $\mathfrak{g}(\wedge x)=\wedge x$. Thus $\wedge x \in \operatorname{Fix}(\mathfrak{g})$, so that $\widehat{K_{G}} \subseteq \operatorname{Fix}(\mathfrak{g})$. Hence $R=F \cup \widehat{K_{G}} \subseteq \operatorname{Fix}(\mathfrak{g}) \subseteq R$. So $R=\operatorname{Fix}(\mathfrak{g})$.

By duality, similarly to Theorem 7.13, we get

Theorem 7.14 (i) dRad is an inscribing set in $\operatorname{Map}(Q)$.

(ii) For each $G \subseteq \mathrm{dRad}$, we have $\operatorname{Fix}\left(\wedge^{\operatorname{Rad}} G\right)=\cup_{g \in G} \operatorname{Fix}(g)$ and

$$
\operatorname{Fix}(\vee G)=\left(\cap_{g \in G} \operatorname{Fix}(g)\right) \cap \stackrel{\vee}{K_{G}} \text {, where } K_{G}=\left\{\left\{x_{g}\right\}_{g \in G}: x_{g} \in \operatorname{Fix}(g)\right\}
$$

(see (7.3)). The set $\operatorname{Fix}(\vee G)$ is the largest inscribing set in $Q$ contained in $\cap_{g \in G} \operatorname{Fix}(g)$.

The subset $\mathcal{T}$ of $\operatorname{Rad}$ of all $T$-radical maps on $Q$ is partially ordered with respect to $\lesssim$. It has the smallest element $\mathbf{0}_{\mathcal{T}}=\mathbf{0}_{\mathrm{Rad}}: x \mapsto x$, and the largest element $\mathbf{1}_{\mathcal{T}}=\mathbf{1}_{\mathrm{Map}}: x \mapsto \mathbf{1}$.

Theorem 7.15 (i) $\mathcal{T}$ is a complete lattice, i.e., $\vee^{\mathcal{T}} G$ and $\wedge^{\mathcal{T}} G$ exist for each $G \subseteq \mathcal{T}$. Moreover,

(a) $\vee^{\mathcal{T}} G=\vee^{\operatorname{Rad}} G=\wedge^{\mathcal{T}} \mathcal{T}^{G}$ (see (7.1)) is the smallest element in $\mathcal{T}^{G}$;

(b) $\wedge^{\mathcal{T}} G=\vee^{\mathcal{T}} \mathcal{T}_{G}$ (see (7.1)) is the largest element in $\mathcal{T}_{G}$;

(c) Fix $\left(\wedge^{\mathcal{T}} G\right)$ is the smallest enveloping sublattice of $Q$ containing $\cup_{g \in G} \operatorname{Fix}(g)$.

(ii) $\mathcal{T}$ is an inscribing sublattice of $\operatorname{Rad}_{Q}$.

Proof. (i) Let $G \subseteq \mathcal{T}$. By Theorem 7.13, there is $\mathfrak{h}=\vee^{\operatorname{Rad}} G$ in $\operatorname{Rad}_{Q}$ and $L:=\operatorname{Fix}(\mathfrak{h})=$ $\cap_{g \in G} \operatorname{Fix}(g)$ is enveloping in $Q$. As each $g \in G$ is $T$-radical, Fix $(g)$ is a sublattice of $Q$. Hence $L$ is an enveloping sublattice of $Q$. By Proposition 7.5, h a $T$-radical map. As $\mathfrak{h}$ is the smallest radical map majorizing all $g \in G$, it is also the smallest $T$-radical map majorizing all $g \in G$, i.e., $\mathfrak{h}=\vee^{\mathcal{T}} G$. Thus $\mathcal{T}$ is $\vee^{\mathcal{T}}$-complete and $\vee^{\mathcal{T}} G=\vee^{\operatorname{Rad}} G$.

As $\mathbf{0}_{\mathcal{T}}$ is the smallest element in $\mathcal{T}$, it follows from Lemma 7.1(iii) that $\mathcal{T}$ is a complete lattice and $\mathfrak{n}$ : $=\wedge^{\mathcal{T}} G=\vee^{\mathcal{T}} \mathcal{T}_{G}$ is the largest element in $\mathcal{T}_{G}$ (see (7.1)). As $\mathcal{T}$ is a complete lattice, Lemma 7.1(i) implies that $\vee^{\mathcal{T}} G=\wedge^{\mathcal{T}} \mathcal{T}^{G}$ is the smallest in $\mathcal{T}^{G}$. This completes the proof of (a) and (b).

(c) As $\mathfrak{n}$ is $T$-radical, $\operatorname{Fix}(\mathfrak{n})$ is an enveloping sublattice of $Q$ by Proposition 7.5. As $\mathfrak{n} \leq g$ for all $g \in G, \operatorname{Fix}(\mathfrak{n})$ contains $\cup_{g \in G} \operatorname{Fix}(g)$ by Lemma 7.12. If $\cup_{g \in G} \operatorname{Fix}(g) \subseteq L$ for some enveloping sublattice $L$, then $L=\operatorname{Fix}\left(f_{L}\right)$ for a $T$-radical map $f_{L}$. By Lemma 7.12, $f_{L} \leq g$ for $g \in G$. Hence $f_{L} \leq \mathfrak{n}$, as $\mathfrak{n}$ is the largest $T$-radical map minorizing all $g \in G$. By Lemma 7.12, Fix $(\mathfrak{n}) \subseteq L$.

(ii) By Theorem 7.13, $\operatorname{Rad}_{Q}$ is a complete lattice. As $\vee^{\mathcal{T}} G=\vee^{\operatorname{Rad}} G$ for all $G \subseteq \mathcal{T}$, $\mathcal{T}$ is a $\vee^{\text {Rad }}$-complete sublattice of $\operatorname{Rad}$. As $\mathbf{0}_{\mathcal{T}} \in \mathcal{T}, \mathcal{T}$ is inscribing in $\operatorname{Rad}$ by Theorem 7.3. 


\subsection{Transfinite extensions of relations}

For $f, g \in \operatorname{Map}(Q)$, their superposition $g \circ f$ is defined by $(g \circ f)(x)=g(f(x))$. If $g$ is a radical map then $f \lesssim g \circ f$. If $f, g$ are radical then $g \circ f$ is radical. Consider the following process of transfinite superposition that has wide applications in the theory of radicals of rings and algebras.

Let $G \subseteq \operatorname{Rad}$. For an interval $[1, \gamma]$ of ordinals, the set $\left(h_{\alpha}\right)_{1 \leq \alpha \leq \gamma}$ in $\operatorname{Rad}$ is an ascending superposition G-series if

$$
\begin{aligned}
& h_{1} \in G, h_{\alpha+1}=g^{\alpha} \circ h_{\alpha} \text { for each } \alpha \in[1, \gamma) \text { and some } g^{\alpha} \in G, \\
& h_{\beta}=\vee_{\alpha<\beta} h_{\alpha} \text { for each limit ordinal } \beta(\text { cf. (4.4)). }
\end{aligned}
$$

If $g \circ h_{\gamma}=h_{\gamma}$ for all $g \in G$, then the series is maximal.

Let $\mathbf{0}_{\operatorname{Rad}} \notin G$. As all $g \in G$ are radical maps, $h_{\alpha+1}$ is larger than $h_{a}$ for all $\alpha$. So the cardinality of $\left(h_{\alpha}\right)_{1 \leq \alpha \leq \gamma}$ is not larger than the cardinality of Rad. Thus each $G$-series extends to a maximal one.

Proposition 7.16 Let $G \subseteq \operatorname{Rad}$ and $L=\cap_{g \in G} \operatorname{Fix}(g)$. Then $L$ is an enveloping set. Moreover,

(i) for each maximal ascending superposition $G$-series $\left(h_{\alpha}\right)_{\alpha \leq \gamma}$,

$$
f_{L}=h_{\gamma}=\vee^{\operatorname{Rad}} G \in \operatorname{Rad}, h_{\gamma}=g \circ h_{\gamma} \text { for all } g \in G \text { and } \operatorname{Fix}\left(h_{\gamma}\right)=L \text {; }
$$

(ii) if $f$ is a radical map and $g \circ f=f$ for all $g \in G$, then $h_{\gamma} \lesssim f$;

(iii) if $x \leq y \in L$ then $h_{\gamma}(x) \leq y$.

Proof. (i) Set $\mathfrak{h}=\vee^{\operatorname{Rad}} G$. By Theorem 7.13, h $\in \operatorname{Rad}$ and $\operatorname{Fix}(\mathfrak{h})=L$. As $\left(h_{\alpha}\right)_{1 \leq \alpha \leq \gamma}$ is maximal, $h_{\gamma}=g \circ h_{\gamma}$ for all $g \in G$. So $h_{\gamma}(x)=g\left(h_{\gamma}(x)\right) \in L$ for all $x \in Q$. Hence $h_{\gamma}(Q) \subseteq L$ and $\operatorname{Fix}\left(h_{\gamma}\right) \subseteq L$.

If $z \in L$ then $g(z)=z$ for all $g \in G$. As $h_{1} \in G, h_{1}(z)=z$ and, by induction, $h_{2}(z)=z, \ldots$, $h_{\gamma}(z)=z$. Thus $L \subseteq \operatorname{Fix}\left(h_{\gamma}\right)$. Hence $L=\operatorname{Fix}\left(h_{\gamma}\right)$ and $h_{\gamma}\left(h_{\gamma}(x)\right)=h_{\gamma}(x)$ for all $x \in Q$.

As $x \leq g(x)$ for all $x \in Q$ and $g \in G$, we have $x \leq h_{1}(x) \leq h_{2}(x)$... By induction, $x \leq h_{\gamma}(x)=$ $h_{\gamma}\left(h_{\gamma}(x)\right)$. If $x \leq y$ then, similarly by induction, $h_{\gamma}(x) \leq h_{\gamma}(y)$. So $h_{\gamma} \in \operatorname{Rad}$. As $\operatorname{Fix}(\mathfrak{h})=L=$ $\operatorname{Fix}\left(h_{\gamma}\right)$, we have $\mathfrak{h}=h_{\gamma}=f_{L}$ by Lemma 7.12 and Proposition 7.5.

(ii) Let $f$ be a radical map and $g \circ f=f$ for all $g \in G$. As $x \leq f(x)$ for all $x \in Q, g(x) \leq$ $g(f(x))=f(x)$ for all $g \in G$. Thus $h_{1} \lesssim f$. Similarly, we prove by induction that $h_{\gamma} \lesssim f$.

(iii) As $h_{\gamma}$ is a radical map, $h_{\gamma}(x) \leq h_{\gamma}(y)$ by $(2.13)$, and $h_{\gamma}(y)=y$ by (i).

By Proposition 7.16, the radical map $f_{L}=\vee^{\operatorname{Rad}} G=h_{\gamma}$ for $L=\cap_{g \in G} \operatorname{Fix}(g)$, is obtained "step by step" via maximal ascending superposition $G$-series. Note that, if for some $\ll$ in $\operatorname{Ref}(Q)$,

$$
g(\ll)=\ll \text { for all } g \in G \text {, then } \ll \text { belongs to } L \text { and } f_{L}(\ll)=\ll \text {. }
$$

By Theorem 7.7, $L_{\mathrm{uc}}, L_{\mathrm{dc}}, L_{\mathrm{ue}}, L_{\mathrm{de}}, L_{\mathrm{o}}$ are enveloping sets in $\operatorname{Ref}(Q)$. So, by Proposition 7.5, $f_{L_{\mathrm{uc}}}, f_{L_{\mathrm{dc}}}, f_{L_{\mathrm{ue}}}, f_{L_{\mathrm{de}}}, f_{L_{\mathrm{o}}}$ are radical maps. Set $G=\left\{f_{L_{\mathrm{uc}}}, f_{L_{\mathrm{dc}}}, f_{L_{\mathrm{ue}}}, f_{L_{\mathrm{de}}}, f_{L_{\mathrm{o}}}\right\}$. By Corollary 7.4, $L=L_{\mathrm{uc}} \cap L_{\mathrm{dc}} \cap L_{\mathrm{ue}} \cap L_{\mathrm{de}} \cap L_{\mathrm{o}}=\cap_{g \in G} \operatorname{Fix}(g)$ is enveloping. As above, $f_{L}=\vee^{\operatorname{Rad}} G=h_{\gamma}$ for any maximal ascending superposition $G$-series $\left(h_{\alpha}\right)_{1 \leq \alpha \leq \gamma}$ (see (7.12)). Set

$$
\widetilde{\ll}=h_{\gamma}(\ll)=f_{L}(\ll) \text { for } \ll \text { from } \operatorname{Ref}(Q) .
$$


Proposition 7.17 For each $\ll$ from $\operatorname{Ref}(Q)$, « is the smallest TT-order majorizing $\ll$.

Proof. By Proposition 7.16, $h_{\gamma} \in \operatorname{Rad}_{\operatorname{Ref}(Q)}$ and $h_{\gamma}=g \circ h_{\gamma}$ for all $g \in G$. Hence $g(\widetilde{\ll})=$ $g\left(h_{\gamma}(\ll)\right)=h_{\gamma}(\ll)=\widetilde{\ll}$ for all $g \in G$. So, by $(7.14), \widetilde{\ll}$ belongs to $L$, so that it is an up- and down-contiguous, and up- and down-expanded order, i.e., it is an TT-order.

If $\prec$ is a TT-order then it is contiguous and expanded (see Definition 2.3). So $\prec$ belongs to $L=\cap_{g \in G} \operatorname{Fix}(g)$. Thus $g(\prec)=\prec$ for all $g \in G$. If $\ll \subseteq \prec($ see $(1.1))$ then $\widetilde{\ll}=h_{\gamma}(\ll) \subseteq \prec$ by Proposition 7.16(iii).

By Theorem 7.7, $L_{\triangleright}, L_{\triangleleft}$ are enveloping sets in $\operatorname{Ref}(Q)$. Hence $G_{\triangleright \triangleleft}=\left\{f_{L_{\triangleright}}, f_{L_{\triangleleft}}\right\}$ consists of radical maps, so that $L_{\triangleright \triangleleft}=L_{\triangleright} \cap L_{\triangleleft}$ is enveloping. The radical map $f_{L}=\vee^{\operatorname{Rad}} G_{\triangleright \triangleleft}$ is obtained by constructing a maximal ascending superposition $G_{\triangleright \triangleleft}$-series $\left(h_{\alpha}\right)_{1 \leq \alpha \leq \gamma}(\operatorname{see}(7.12))$ and $f_{L_{\triangleright \triangleleft}}=h_{\gamma}$. For $\ll \operatorname{from} \operatorname{Ref}(Q)$, set

$$
\bar{\ll}=h_{\gamma}(\ll)=f_{L_{\triangleright \triangleleft}}(\ll) .
$$

Example 7.18 The relation $\ll$ in $Q$ can be neither contiguous, nor expanded. Indeed,

$1)$ Let $Q=\{\mathbf{0}, a, \mathbf{1}\}, \mathbf{0}<a<\mathbf{1}$ and $\mathbf{0} \ll \mathbf{1}$. Then $\ll=\ll$ is not contiguous.

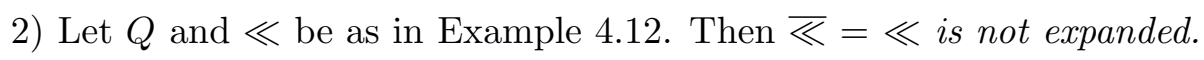

Proposition 7.19 (i) $\ll=\ll=\nwarrow^{\triangleleft}$ and $\ll$ is the smallest of all relations $\prec$ satisfying $\ll \subseteq \prec$ $=\prec^{\triangleright}=\prec^{\triangleleft}$.

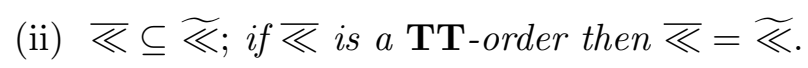

(iii) If $\ll$ is an expanded order then $\ll=\ll$.

(iv) Let $Q$ be a chain. If $\ll$ is up- or down-contiguous, « is up-or down-contiguous, respectively.

Proof. The proof of (i) is the same as in Proposition 7.17 .

(ii) As $\widetilde{\ll}$ is a TT-order, $\ll \subseteq \widetilde{\ll}=\widetilde{\ll}=\widetilde{\ll}$ by Theorem 4.9 . Hence $\ll \subseteq \widetilde{\ll}$ by (i).

By Proposition $7.17, \widetilde{\ll}$ is the smallest $\mathbf{T T}$-order majorizing $\ll$. So, if $\ll$ is a $\mathbf{T T}$-order then $\widetilde{\ll} \ll$. Thus $\bar{\ll}=\widetilde{\ll}$.

(iii) If $\ll$ is an expanded order, it follows from Theorem 4.9 that $\ll=\ll^{\triangleleft}=\ll^{\triangleright}$. Thus $g(\ll)=$ $\ll$ for all $g \in G_{\triangleright \triangleleft}$. By $(7.14), \ll=h_{\gamma}(\ll)=\ll$.

(iv) If $Q$ is a chain then, by Lemma 4.11 , if $\ll$ is up-contiguous, the relations $\ll^{\triangleleft}$ and $\ll^{\triangleright}$ are up-contiguous. By induction, $\ll$ is up-contiguous. The down-contiguous case is similar.

By Proposition $7.19, \ll \subseteq \widetilde{\ll}$. Below we consider the case when they coincide.

Proposition 7.20 Let $Q$ have properties (JIDC) and (MIDC) (see Definition 6.8). If $\ll$ is an

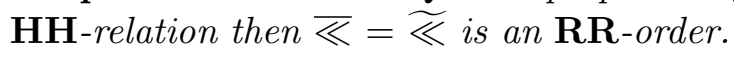

Proof. Let $Q$ have properties (JIDC) and (MIDC) and let $G_{\triangleright \triangleleft}=\left\{f_{L_{\triangleright}}, f_{L_{\triangleleft}}\right\}$. By Lemma 7.9, the radical maps $f_{L_{\triangleright}}, f_{L_{\triangleleft}}$ on $Q$ act by $f_{L_{\triangleright}}(\ll)=\ll^{\triangleright}$ and $f_{L_{\triangleleft}}(\ll)=\ll^{\triangleleft}$. Let $\ll$ be an HH-relation. By Lemma $6.9, \ll^{\triangleleft}$ is an $\mathbf{H}$-order and a dual $\mathbf{R}$-order, and $\ll^{\triangleright}$ is an $\mathbf{R}$-order and a dual $\mathbf{H}$-order. So $f_{L_{\triangleleft}}(\ll)=\ll^{\triangleleft}$ and $f_{L_{\triangleright}}(\ll)=\ll^{\triangleright}$ are $\mathbf{H H}$-orders.

Let $\left(h_{\alpha}\right)_{1 \leq \alpha \leq \gamma}$ be a maximal ascending superposition $G_{\triangleright \triangleleft}$-series Set $\ll_{\alpha}=h_{\alpha}(\ll)$. If $\ll_{\alpha}$ is an HH-order, then $\ll_{\alpha+1}$ is either $f_{L_{\triangleright}}\left(\ll_{\alpha}\right)$, or $f_{L_{\triangleleft}}\left(\ll_{\alpha}\right)$, so that it is an HH-order by above. 
Let $\beta$ be a limit ordinal. By (7.12), $\ll_{\beta}=h_{\beta}(\ll) \stackrel{(7.12)}{=} \vee_{\alpha<\beta} h_{\alpha}(\ll)=\vee_{\alpha<\beta} \ll_{\alpha}$. If $a \ll_{\beta} b$ in $Q$, it follows from (5.6) that $a \ll_{\alpha} b$ for some $\alpha<\beta$. As $\ll_{\alpha}$ is an HH-order, $a \wedge z \ll_{\alpha} b \wedge z$ and $a \vee z \ll_{\alpha} b \vee z$ for all $z \in Q$. Hence, by (5.6), $a \wedge z \ll_{\beta} b \wedge z$ and $a \vee z \ll_{\beta} b \vee z$. Thus $\ll_{\beta}$ is an $\mathbf{H H}$-order. So, by induction, $\ll=h_{\gamma}(\ll)$ is an $\mathbf{H H}$-order.

By $(7.13), \widetilde{\ll}^{\triangleleft}=f_{L_{\triangleleft}}(\widetilde{\ll})=f_{L_{\triangleleft}}\left(h_{\gamma}(\ll)\right)=h_{\gamma}(\ll)=\widetilde{\ll}$ and $\ll^{\triangleright}=f_{L_{\triangleright}}(\widetilde{\ll})=f_{L_{\triangleright}}\left(h_{\gamma}(\ll)\right)=$ $h_{\gamma}(\ll)=\ll$. Then, by Corollary 5.6, $\ll$ is an RR-order. So $\ll$ is a TT-order. By Proposition $7.19, \ll=\widetilde{\ll}$.

Corollary 7.21 Let a modular complete lattice $Q$ have properties (JIDC) and (MIDC) (in particular, $Q$ is a complete chain). Let $<_{\mathfrak{g}}$ and $<_{\mathfrak{c}}$ be the relations in $Q$ defined in Definition 6.1. Then

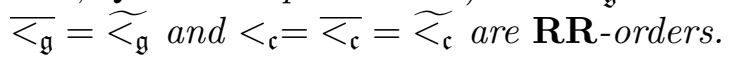

Proof. By Corollary 6.7, $<_{\mathfrak{g}}$ is an $\mathbf{H H}$-relation, as $Q$ is modular. Since $Q$ has properties (JIDC) and (MIDC), Proposition 7.20 implies that $\overline{\overline{<}_{\mathfrak{g}}}=\widetilde{\overline{<}_{\mathfrak{g}}}$ is an RR-order.

By Theorem $6.10, \leq_{\mathfrak{c}}$ is an $\mathbf{R R}$-order, as $Q$ is modular and has properties (JIDC) and (MIDC). Hence $<_{\mathfrak{c}}=<_{\mathfrak{c}}^{\triangleleft}=<_{\mathfrak{c}}$ by Corollary 5.6. So $<_{\mathfrak{c}}={\overline{<_{\mathfrak{c}}}}_{\text {by }}$ (7.14). It also follows from Proposition 7.20 that $\bar{\ll}_{\mathfrak{c}}=\widetilde{\ll_{\mathfrak{c}}}$.

Problem 7.22 Let a modular lattice do not have properties (JIDC) and (MIDC). Will $\overline{<_{\mathfrak{g}}}=\widetilde{\overline{\mathrm{q}}_{\mathfrak{g}}}$ and $<_{\mathfrak{c}}=\overline{<_{\mathfrak{c}}}=\widetilde{<_{\mathfrak{c}}}$ ? Will they still be $\mathbf{R R}$-orders?

Let $\ll$ belong to $\operatorname{Ref}(Q)$. We say that a chain $C$ in $Q$ is complete $\ll-g a p$ dense if,

for all $z<w$ in $C$ there are $u \ll v$ in $[z, w]_{C}$ such that $[u, v]_{C}$ is a gap in $C$.

Consider the following reflexive relation $\ll_{\mathrm{g} \text {-d }}$ on $Q$ : for $a<b$, we write

$$
a \ll_{\text {g-d }} b \text { if there is a complete } \ll \text {-gap dense chain from } a \text { to } b \text {. }
$$

Example 7.23 The relation $\ll_{\mathrm{g}-\mathrm{d}}$ in $Q$ can be neither contiguous, nor expanded. Indeed,

1) Let $Q=\{\mathbf{0}, a, b, \mathbf{1}\}, \mathbf{0}<a<\mathbf{1}, \mathbf{0}<b<\mathbf{1}$. Let $\ll$ be a reflexive order in $Q$ such that $\mathbf{0} \ll a \ll \mathbf{1}$. Then $\ll_{\mathrm{g}-\mathrm{d}}=\ll$ and $\mathbf{0} \ll_{\mathrm{g}-\mathrm{d}} \mathbf{1}$. However $[\mathbf{0}, \mathbf{1}] \nsubseteq\left[\ll_{\mathrm{g}-\mathrm{d}}, \mathbf{1}\right]$. So $\ll_{\mathrm{g}-\mathrm{d}}$ is not contiguous.

$2)$ Let $\ll$ be the relation considered in Example 4.12. Then $\ll_{\mathrm{g}-\mathrm{d}}=\ll$ is not expanded.

Note that $\ll_{\text {g-d }}$ is an order. Each complete upper (lower) «-gap chain is «-gap dense.

The proof of the following lemma is evident.

Lemma 7.24 Let $T$ be a chain from a to $b$ such that each $t \in T \backslash\{b\}$ has an immediate successor $t_{s}$ in $T$, i.e., $\left[t, t_{s}\right]_{T}=\left\{t, t_{s}\right\}$ is a gap in $T$. Let $\ll$ belong to $\operatorname{Ref}(Q)$ and suppose that, for each $t \in T \backslash\{b\}$, there is a -gap dense chain $C_{t}$ in $Q$ from $t$ to $t_{s}$. Set $C_{b}=\{b\}$. Then the chain $C=\cup_{t \in T} C_{t}$ is «-gap dense.

Corollary 7.25 (i) For each $\ll$ from $\operatorname{Ref}(Q), \ll \subseteq \ll_{\text {g-d }}=\left(\ll_{\text {g-d }}\right)^{\triangleright}=\left(\ll_{\text {g-d }}\right)^{\triangleleft}$. So $\ll_{\text {g-d }}=\ll_{\text {g-d }}$.

(ii) For each $\ll$ from $\operatorname{Ref}(Q), \ll \subseteq \ll_{\text {g-d }}$. 
Proof. (i) Clearly, $\ll \subseteq \ll_{\text {g-d }}$ and $\ll_{\text {g-d }}$ is an order. Set $\prec=\ll_{\text {g-d }}$. If $a \prec^{\triangleright} b$ then there is a complete upper $\prec$-gap chain $T$ from $a$ to $b$, i.e., each $t \in T \backslash\{b\}$ has the immediate $\prec$-successor $t_{s}$ in $T$. Then there is a complete $\ll$-gap dense chain $C^{t}$ in $Q$ from $t$ to $t_{s}$. Set $C^{b}=\{b\}$. By Lemma 7.24, $C=\cup_{t \in T} C^{t}$ is a complete «-gap dense chain from $a$ to $b$. Thus $a \prec b$, so that $\prec \triangleright \prec$. As $\prec \subseteq \prec$ by (4.7), we have $\prec^{\triangleright}=\prec$. Similarly, $\prec^{\triangleleft}=\prec$. By $(7.14), \bar{\prec}=\prec$.

(ii) By Proposition 7.19(i), « is the smallest of all relations $\prec$ satisfying the condition $\ll \subseteq \prec$ $=\prec^{\triangleright}=\prec^{\triangleleft}$. As the relation $\ll_{\text {g-d }}$ satisfies this condition by (i), $\ll \subseteq \ll_{\text {g-d }}$.

In general, $\ll \neq \ll_{\mathrm{g} \text {-d }}$ in $Q$, even if $Q$ is a chain. To show this, consider the gap relation $<_{\mathfrak{g}}$ (see Definition 6.1). First, it should be noted that, although the relations $<_{\mathfrak{g}}$ and $<_{\mathfrak{c}}$ are not compatible (if $a<_{\mathfrak{c}} b$ then $a \nless_{\mathfrak{g}} b$ and vice versa), the relations ${\overline{<_{\mathfrak{g}}}}_{\text {and }}<_{\mathfrak{c}}$ can be compatible, as the lattice $Q$

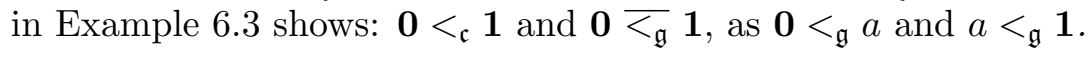

Proposition 7.26 If $Q$ has no continuous chains (see Definition 6.1 ) then $\left(<_{\mathfrak{g}}\right)_{\mathrm{g}-\mathrm{d}}=\leq$.

Proof. For $a<b$ in $Q$, let $C$ be a maximal chain from $a$ to $b$. By Lemma 4.5, $C$ is complete. By (7.18), $C$ is $<_{\mathfrak{g}}$-gap dense if

$$
\text { each interval }[x, y]_{C} \subseteq C \text { contains a gap }[u, v]_{Q} .
$$

Hence, if $C$ is not $\ll_{\mathfrak{g}}$-gap dense, then there is an interval $[x, y]_{C}$ in $C$ without gaps. So, by Definition $6.1,[x, y]_{C}$ is a continuous chain, a contradiction. Thus $C$ is $<_{\mathfrak{g}^{-}}$gap dense, so that $a\left(<_{\mathfrak{g}}\right)_{\mathrm{g}-\mathrm{d}} b$. Hence $\leq \subseteq\left(<_{\mathfrak{g}}\right)_{\mathrm{g}-\mathrm{d}}$. As we always have $\left(<_{\mathfrak{g}}\right)_{\mathrm{g}-\mathrm{d}} \subseteq \leq$, so $\left(<_{\mathfrak{g}}\right)_{\mathrm{g}-\mathrm{d}}=\leq$.

We shall now consider an example of a chain, for which $\overline{<_{\mathfrak{g}}} \neq\left(<_{\mathfrak{g}}\right)_{\mathrm{g}-\mathrm{d}}$.

Example 7.27 Let $Q=\{(t ; n): t \in[0,1] \subset \mathbb{R}, n=0,1\}$. Let $(t ; n)<(s ; m)$, if either $t<s$, or $t=s, n=0$ and $m=1$. Then $Q$ is a complete chain from $\mathbf{0}=(0 ; 0)$ to $\mathbf{1}=(1 ; 1)$.

As each interval $[(t, 0),(t, 1)]$ in $Q$ is a gap, all intervals in $Q$ have gaps. So, by $(7.19), Q$ is $<_{\mathfrak{g}}$-gap dense. By Proposition 7.26, $\left(<_{\mathfrak{g}}\right)_{\mathfrak{g} \text {-d }}=\leq$. Hence $\mathbf{0}\left(<_{\mathfrak{g}}\right)_{\mathrm{g} \text {-d }} \mathbf{1}$.

On the hand, it is easy to see that $<_{\mathfrak{g}}={\overline{<_{\mathfrak{g}}}}$. So $\mathbf{0} \nless_{\mathfrak{g}} \mathbf{1}$. Thus $\overline{<_{\mathfrak{g}}} \neq\left(<_{\mathfrak{g}}\right)_{\mathrm{g} \text {-d }}$.

Although the relations $\ll$ and $\ll_{\text {g-d }}$ do not coincide, they are closely linked.

Theorem 7.28 If $a \ll b$ then there is a complete «-gap dense chain $C$ from a to $b$ such that $x$ $\ll y$ for all $x<y$ in $C$.

Proof. Let $G_{\triangleright \triangleleft}=\left\{f_{L_{\triangleright}}, f_{L_{\triangleleft}}\right\}$ and $\left(h_{\alpha}\right)_{1 \leq \alpha \leq \gamma}$ be a maximal ascending superposition $G_{\triangleright \triangleleft}$-series, $h_{1}=f_{L_{\triangleleft}}$ and $h_{\gamma}=f_{L_{\triangleright \triangleleft}}$. Set $\ll_{\alpha}=h_{\alpha}(\ll)$ and $\bar{\ll}=\ll_{\gamma}$. Consider the following induction.

Let $a \ll_{1} b$, i.e., $a \ll^{\triangleleft} b$ for some $a, b \in Q$. Then there is a complete lower $\ll$-gap chain $C^{1}$ from $a$ to $b$. As each $x \in C^{1} \backslash\{a\}$ has an immediate «-predecessor $x_{p}$ : $\left[x_{p}, x\right]_{C}$ is a gap in $C^{1}$ and $x_{p} \ll x$. So $C^{1}$ is «-gap dense. For all $x<y$ in $C^{1},[x, y]_{C^{1}}$ is a complete lower «-gap chain from $x$ to $y$. Thus $x \ll^{\triangleleft} y$.

Assume that if $a \ll_{\alpha} b$, for some $a, b \in Q$ and some $\alpha<\gamma$, then

1) there is a complete $\ll$-gap dense chain $C^{\alpha}$ from $a$ to $b$ and

2) $x \ll_{\alpha} y$ for all $x<y$ in $C^{\alpha}$. 
Let now $a \ll_{\alpha+1} b$, say, $a\left(\ll_{\alpha}\right)^{\triangleright} b$ for some $a, b \in Q$. Then there is a complete upper gap $\ll_{\alpha^{-}}$ chain $T$ from $a$ to $b$, i.e., each $t \in T \backslash\{b\}$ has an immediate $\ll_{\alpha}$-successor $t_{s} \in T-\left[t, t_{s}\right]_{T}=\left\{t, t_{s}\right\}$ is a gap in $T$ and $t \ll_{\alpha} t_{s}$. By (7.20), for each $t \in T \backslash\{b\}$, there is a complete $\ll$-gap dense chain $C_{t}$ from $t$ to $t_{s}$ satisfying (7.21). Set $C_{b}=\{b\}$. By Lemma 7.24, $C^{\alpha+1}=\cup_{t \in T} C_{t}$ is a complete $\ll$-gap dense chain from $a$ to $b$.

Let $x<y$ in $C^{\alpha+1}$. Then $x \in C_{t}, y \in C_{t^{\prime}}$ for some $t \leq t^{\prime}$ in $T$. If $t=t^{\prime}$ then $x, y \in C_{t}$ and $x \ll_{\alpha} y$, by (7.21). Thus $x \ll_{\alpha+1} y$ by (4.7). If $t \neq t^{\prime}$ then either $x<t_{s}<y$, or $x=t_{s}<y$, or $x<t_{s}=y$. Let $x<t_{s}<y$. As $C_{t}, C_{t^{\prime}}$ satisfy (7.21), $x \ll_{\alpha} t_{s}$ and $t^{\prime} \ll_{\alpha} y$. Hence $x \ll_{\alpha+1} t_{s}$ and $t^{\prime} \ll_{\alpha+1} y$ by (4.7). As $T$ is a complete upper gap $\ll_{\alpha}$-chain, $t_{s} \ll_{\alpha+1} t^{\prime}$. As $\ll_{\alpha+1}$ is an order, $x \ll_{\alpha+1} y$. Similarly, $x \ll_{\alpha+1} y$ when $x=t_{s}<y$, or $x<t_{s}=y$. Thus (7.21) holds for all $x<y$ in $C^{\alpha+1}$ and $\ll_{\alpha+1}$.

Let $\beta$ be a limit ordinal and let, for each $\alpha<\beta, a \ll_{\alpha} b$ imply (7.20) and (7.21). Let now $a \ll_{\beta} b$. As $\ll_{\beta}=h_{\beta}(\ll) \stackrel{(7.12)}{=} \vee_{\alpha<\beta} h_{\alpha}(\ll)=\vee_{\alpha<\beta} \ll_{\alpha}$, it follows from (5.6) that $a \ll_{\alpha} b$ for some $\alpha<\beta$. Hence there is a complete $\ll$-gap dense chain $C^{\alpha}$ from $a$ to $b$ satisfying (7.21). Set $C^{\beta}=C^{\alpha}$. Then $C^{\beta}$ satisfies (7.20). Let $x<y$ in $C^{\beta}$. By (7.21), $x \ll_{\alpha} y$. Hence, by (5.6), $x \ll_{\beta} y$. Thus (7.20) and (7.21) hold for $\beta$. By induction, if $a \ll_{\gamma} b$ then there is a complete $\ll$-gap dense chain $C^{\gamma}$ from $a$ to $b$ satisfying $(7.21)$ for $\alpha=\gamma$. As $\ll_{\gamma}=\ll$, the proof is complete.

\section{Relations in lattices of subspaces of Banach spaces}

Let $X$ be a Banach space. The set $\operatorname{Ln}(X)$ of all linear subspaces of $X$ and the set $\mathrm{Cl}(X)$ of all closed subspaces of $X$ are complete lattices with order $\subseteq$,

$$
\begin{gathered}
\mathbf{0}=\{0\}, \mathbf{1}=X, \wedge G=\bigcap_{Y \in G} Y \text { for a subset } G \neq \varnothing \text { in } \operatorname{Ln}(X) \text { and } \mathrm{Cl}(X), \\
\vee G=\sum_{Y \in G} Y \text { for } G \subseteq \operatorname{Ln}(X) \text { and } \vee G=\overline{\sum_{Y \in G} Y} \text { for } G \subseteq \operatorname{Cl}(X) .
\end{gathered}
$$

As before, for $L \subseteq M$ in $Q$, we write

$$
L<_{\mathfrak{g}} M \text { if either } L=M \text {, or }[L, M]_{Q} \text { is a gap in } Q .
$$

In this section we concentrate on the relation $<_{\mathfrak{g}}$ in the sublattices of $\operatorname{Ln}(X)$ and $\mathrm{Cl}(X)$. We show that all sublattices of $\operatorname{Ln}(X)$ are modular, so that $<_{\mathfrak{g}}$ is an HH-relation in all of them. The lattice $\mathrm{Cl}(X)$ is not modular. Although $<_{\mathfrak{g}}$ is an $\mathbf{H H}$-relation in many of its sublattices (Corollary 8.24), there are sublattices of $\mathrm{Cl}(X)$, where it is neither an $\mathbf{H}$-, nor a dual $\mathbf{H}$-relation (Corollary 8.21).

Let $Q$ be a complete sublattice of $\operatorname{Ln}(X)$, or $\operatorname{Cl}(X)$. As in $(2.2)$ and (5.7), for $\ll$ from $\operatorname{Ref}(Q)$,

$$
\begin{array}{r}
{[\ll, L]_{Q}=\{K \in Q: K \ll L\},[L, \ll]_{Q}=\{K \in Q: L \ll K\} \text { for } L \in Q ;} \\
\sigma_{\ll}(L)=\wedge[\ll, L]_{Q}=\cap\left\{K: K \in[\ll, L]_{Q}\right\} \text { and } s^{\ll}(L)=\vee[L, \ll]_{Q}, \text { so that } \\
s^{\ll}(L)=\sum K \in[L, \ll]_{Q} K \text { in } \operatorname{Ln}(X), \text { and } s^{\ll}(L)=\overline{\sum K \in[L, \ll]_{Q} K} \text { in } \operatorname{Cl}(X) .
\end{array}
$$

It follows from Theorem 5.9 that if $\ll$ is an $\mathbf{H}$-relation then $\ll^{\triangleright}$ is an $\mathbf{R}$-order in $Q$ and $\mathfrak{r}_{L}=s^{\ll^{\triangleright}}(L)$ is the unique $\ll^{\triangleright}$-radical in $[L, X]_{Q}$, i.e., $L \ll \mathfrak{r}_{L} \overleftarrow{\ll} X$. 
If $\ll$ is a dual $\mathbf{H}$-relation then $\ll^{\triangleleft}$ is a dual $\mathbf{R}$-order in $Q$ and $\mathfrak{p}_{L}=\sigma_{\ll^{\triangleleft}}(L)$ is the unique dual $\ll^{\triangleleft}$-radical in $[\{0\}, L]_{Q}$, i.e., $\{0\} \gtrless \mathfrak{p}_{L} \ll^{\triangleleft} L$. By $(5.8)$ and $(5.9)$,

$$
s^{\ll}(L) \subseteq \mathfrak{r}_{L} \text { for an } \mathbf{H} \text {-relation } \ll \text {, and } \mathfrak{p}_{L} \subseteq \sigma_{\ll}(L) \text { for a dual H-relation } \ll \text {. }
$$

Thus, for a complete sublattice $Q$ of $\operatorname{Ln}(X)$, or of $\mathrm{Cl}(X)$, Theorem 5.9 yields

Theorem 8.1 (i) Let $\ll$ be an $\mathbf{H}$-relation and $L \subseteq K$ in $Q$.

1) If $K \subseteq \mathfrak{r}_{L}$ then there is an ascending «-series of spaces in $Q$ from $K$ to $\mathfrak{r}_{L}$.

2) If $\mathfrak{r}_{L} \nsubseteq K \neq X$ then $K$ has a $\ll$-successor $S \in Q: K \ll S ; \mathfrak{r}_{L}$ has no $\ll$-successor.

3) If there is an ascending «-series of spaces in $Q$ from $L$ to $K$ then $K \subseteq \mathfrak{r}_{L}$.

(ii) Let $\ll$ be a dual $\mathbf{H}$-relation and $K \subseteq L$ in $Q$.

1) If $\mathfrak{p}_{L} \subseteq K$ then there is a descending $\ll$-series of spaces in $Q$ from $K$ to $\mathfrak{p}_{L}$.

2) If $\{0\} \neq K \nsubseteq \mathfrak{p}_{L}$ then $K$ has a $\ll$-predecessor $P \in Q: P \ll K ; \mathfrak{p}_{L}$ has no $\ll$-predecessor.

3) If there is a descending «-series of spaces in $Q$ from $L$ to $K$ then $\mathfrak{p}_{L} \subseteq K$.

If $X$ is separable then the $\ll$-series in Theorem 8.1 are isomorphic to $\mathbb{N}$.

Proposition 8.2 Let $X$ be a separable Banach space and $Q$ be a complete sublattice of $\mathrm{Cl}(X)$.

(i) If $\ll$ is a dual $\mathbf{H}$-relation in $Q$ then there are spaces

$$
\ldots \ll Y_{n} \ll \ldots \ll Y_{1} \ll X \text { in } Q \text { such that } \cap_{n=1}^{\infty} Y_{n}=\sigma_{\ll}(X) \text {. }
$$

There are also spaces $\ldots \ll^{\triangleleft} Z_{n} \ll^{\triangleleft} \ldots \ll^{\triangleleft} Z_{1} \ll^{\triangleleft} X$ in $Q$ such that

$$
\cap_{n=1}^{\infty} Z_{n}=\mathfrak{p}_{X} \text { is the dual } \ll^{\triangleleft} \text {-radical in } X \text {. }
$$

(ii) If $\ll$ is an $\mathbf{H}$-relation then there are spaces $\{0\} \ll Y_{1} \ll \ldots \ll Y_{n} \ll \ldots$ in $Q$ and spaces $\{0\} \ll^{\triangleright} Z_{1} \ll \ll^{\triangleright} Z_{n} \ll$.. in $Q$ such that

$$
\overline{\sum_{n=1}^{\infty} Y_{n}}=s^{\ll}(\{0\}) \text { and } \overline{\sum_{n=1}^{\infty} Z_{n}}=\mathfrak{r}_{\{0\}} \text { is the } \ll^{\triangleright} \text {-radical in } X \text {. }
$$

Proof. (i) Set $\omega=\sigma_{\ll}(X)$. Let $\mathbf{B}$ be the unit ball of $X^{*}$. For each $L \in[\ll, X]_{Q}$, set $W_{L}=\{f \in$ B : $L \subseteq \operatorname{ker}(f)\}$. Set $W=\cup_{L \ll X} W_{L}$. Then $W \subseteq \mathbf{B}$. As $X$ is separable, $\mathbf{B}$ is a separable metric space in the $\sigma\left(X^{*}, X\right)$-topology (see [Sch, Section 4.1.7]). Hence $W$ has a $\sigma\left(X^{*}, X\right)$ dense sequence $\left\{f_{k}: k \in \mathbb{N}\right\}$. As each $L=\cap_{f \in W_{L}} \operatorname{ker} f$, we have $\cap_{k} \operatorname{ker}\left(f_{k}\right)=\cap_{f \in W} \operatorname{ker}(f)=\cap_{L \ll X} L=\omega$.

For each $k \in \mathbb{N}$, choose $L_{k} \in[\ll, X]_{Q}$ such that $f_{k} \in W_{L_{k}}$. Then, by (8.4), $\omega \subseteq \cap_{k=1}^{\infty} L_{k} \subseteq$ $\cap_{k} \operatorname{ker}\left(f_{k}\right)=\omega$. Hence $\cap_{k=1}^{\infty} L_{k}=\omega$.

Set $Y_{n}=L_{1} \cap \ldots \cap L_{n} \in Q$. Then $Y_{k+1} \subseteq Y_{k}$ for all $k$, and $\cap_{n=1}^{\infty} Y_{n}=\cap_{k=1}^{\infty} L_{k}=\omega$. Suppose, by induction, that $Y_{k} \ll Y_{k-1} \ll \ldots \ll Y_{1} \ll X$ for some $k$. As all $L_{n} \in[\ll, X]_{Q}$, we have $L_{k+1} \ll X$. Since $\ll$ is a dual $\mathbf{H}$-order, it follows from Lemma 3.1(ii) that

$$
Y_{k+1}=Y_{k} \cap L_{k+1}=Y_{k} \wedge L_{k+1} \ll Y_{k} \wedge X=Y_{k} \cap X=Y_{k} .
$$

Hence (8.6) holds. As $\mathfrak{p}_{X}=\sigma_{\ll^{\triangleleft}}(X)$ and $\ll^{\triangleleft}$ is a dual R-order in $Q$, (8.7) follows from (8.6).

Part (ii) can be proved similarly. 


\subsection{Relations $<_{\mathfrak{g}}$ and $\ll_{n}$ in sublattices of $\operatorname{Ln}(X)$.}

Proposition 8.3 $\operatorname{Ln}(X)$ is modular and $<_{\mathfrak{g}}$ is an $\mathbf{H H}$-relation in $\operatorname{Ln}(X)$.

Proof. Let $I, J, K \in \operatorname{Ln}(X)$ and $I \subseteq J$. Then $\curlyvee_{I} \curlywedge_{J}(K)=I+(J \cap K)$ and $\curlywedge_{J} \curlyvee_{I}(K)=$ $J \cap(I+K)$. Clearly, $I+(J \cap K) \subseteq J \cap(I+K)$. Conversely, if $x \in J \cap(I+K)$ then $x=i+k \in J$, where $i \in I, k \in K$. Then $i \in J$, as $I \subseteq J$. Hence $k \in J \cap K$. So $x \in I+(J \cap K)$. Thus $J \cap(I+K) \subseteq I+(J \cap K)$. Hence $I+(J \cap K)=J \cap(I+K)$. So $\curlyvee_{I} \curlywedge_{J}(K)=\curlywedge_{J} \curlyvee_{I}(K)$. Thus $\operatorname{Ln}(X)$ is modular by $(6.2)$.

It follows from Corollary 6.7 that $<_{\mathfrak{g}}$ is an HH-relation in $\operatorname{Ln}(X)$.

To introduce more HH-relations, we will use the following result proved in Theorem 2.2 [Di].

Lemma 8.4 Let $L \subset M$ in $\operatorname{Ln}(X)$ and $n:=\operatorname{dim}(M / L)<\infty$. Let $K \in \operatorname{Ln}(X)$.

(i) If $K \subseteq M$ then $\operatorname{dim}(K /(L \cap K))=\operatorname{dim}((L+K) / L) \leq n$.

(ii) If $L \subseteq K$ then $\operatorname{dim}(M /(M \cap K))=\operatorname{dim}((M+K) / K) \leq n$.

(iii) If $K, L, M$ are closed subspaces, then $L+K$ in (i) and $M+K$ in (ii) are closed.

Consider the following relation in $\operatorname{Ln}(X)$. For $n \in \mathbb{N} \cup \infty$ and $L \subseteq M$ in $\operatorname{Ln}(X)$, we write

$$
L \ll_{n} M \text { if } \operatorname{dim}(M / L)<n .
$$

Corollary 8.5 All $\ll_{n}$ are $\mathbf{H H}$-relations in each sublattice of $\operatorname{Ln}(X)$ and $\ll_{\infty}$ is an $\mathbf{H H}$-order.

Proof. Let $L \ll_{n} M$, i.e., $\operatorname{dim}(M / L)<n$. Let $L \subseteq K$. By (8.1) and Lemma 8.4(ii), $M \vee K=$ $M+K$ and $\operatorname{dim}((M+K) / K) \leq n$. Hence $K \ll_{n} M \vee K$. So, by Lemma 3.1(i), $\ll_{n}$ is an H-relation.

Let $K \subseteq M$. By (8.1) and Lemma 8.4(i), $L \wedge K=L \cap K$ and $\operatorname{dim}(K /(L \cap K)) \leq n$. Hence $L \wedge K \ll_{n} K$. So, by Lemma 3.1(ii), $\ll_{n}$ is a dual $\mathbf{H}$-relation. Clearly, $\ll_{\infty}$ is an $\mathbf{H H}$-order.

\subsection{Relations $\ll_{n},<_{\mathfrak{g}}, \sqsubset_{\mathfrak{g}}$ and $\prec_{\mathfrak{g}}$ in sublattices of $\mathrm{Cl}(X)$.}

Note that the restrictions of $\mathbf{H}$ - and dual $\mathbf{H}$-relations to sublattices $Q$ of $\mathrm{Cl}(X)$ also have the same properties. However, an $\mathbf{H}$-, or a dual $\mathbf{H}$-relation in $Q$ is not necessarily a restriction of a relation in $\mathrm{Cl}(X)$ with the same property.

Making use of Lemma 8.4(iii), and repeating the proof of Corollary 8.5, we get

Corollary 8.6 $\left\{\ll_{n}\right\}_{n=1}^{\infty}$ are $\mathbf{H H}$-relations in all sublattices of $\mathrm{Cl}(X)$ and $\ll_{\infty}$ is an $\mathbf{H H}$-order.

The relation $<_{\mathfrak{g}}$ is an $\mathbf{H H}$-relation in many sublattices of $\mathrm{Cl}(X)$. Corollary 6.11 yields

Lemma 8.7 If $Q$ is a nest (a complete linearly ordered set) in $\mathrm{Cl}(X)$ then $<_{\mathfrak{g}}$ is an $\mathbf{H H}$-relation.

However, there are sublattices of $\mathrm{Cl}(X)$, where $<_{\mathfrak{g}}$ is neither an $\mathbf{H}$-, nor a dual $\mathbf{H}$-relation (see Corollary 8.21). The main obstacle is the fact that the sum of subspaces is not necessarily closed.

To avoid this, introduce relations $\sqsubset$ and $\prec$ in sublattices $Q$ of $\mathrm{Cl}(X)$. For $L \subseteq M$ in $Q$, we write

$$
\begin{aligned}
& L \sqsubset M \text { if } M+K \text { is closed for each } K \in Q \text { such that } L \subseteq K, \\
& L \prec M \text { if } L+K \text { is closed for each } K \in Q \text { such that } K \subseteq M .
\end{aligned}
$$

We will show that the intersection of $<_{\mathfrak{g}}$ with $\sqsubset$ is an $\mathbf{H}$-relation and with $\prec$ is a dual $\mathbf{H}$-relation. 
Proposition $8.8 \sqsubset$ is an $\mathbf{H}$-relation and $\prec$ is a dual $\mathbf{H}$-relation in any sublattice $Q$ of $\mathrm{Cl}(X)$.

Proof. Let $L \sqsubset M$ in $Q$. For $L \subseteq K \in Q$, let us show that $K \sqsubset M \vee K$. Indeed, for each $R \in Q, K \subseteq R$, we have $L \subseteq R$, so that $(M \vee K)+R=M+K+R=M+R$ is closed. Thus $K \sqsubset M \vee K$. Hence $\sqsubset$ is an $\mathbf{H}$-relation by Lemma 3.1(i).

Let $L \prec M$ in $Q$. For $K \subseteq M$ in $Q$, let us show that $L \cap K \prec K$. Indeed, for each $R \in Q$, $R \subseteq K$, we have $R \subseteq M$, so that $L \cap K+R=(L+R) \cap K$ is closed, as $L+R$ is closed. Thus $L \cap K \prec K$. Hence $\prec$ is a dual $\mathbf{H}$-relation by Lemma 3.1(ii).

In many important sublattices $Q$ of $\mathrm{Cl}(X)$ the relations $\sqsubset, \prec, \subseteq$ coincide. For example, they coincide if $Q$ is a nest (a linearly ordered set of subspaces), or a commutative subspace lattice, if $X$ is a Hilbert space (Theorem 8.23).

Consider another example. Let $B(X)$ the algebra of all bounded operators on $X$. A projection $p$ in $B(X)$ is an $L$-projection if $\|x\|=\|p x\|+\|x-p x\|$ for $x \in X$. The subspace $p X$ of $X$ is called an $L$-summand. A subspace $J$ of $X$ is an $M$-ideal if

$$
J^{\perp}=\left\{f \in X^{*}: f(x)=0 \text { for all } x \in J\right\} \text { is an } L \text {-summand in } X^{*} .
$$

Some spaces (for example, $L^{q}$-spaces, $1<q<\infty$ ) have no non-trivial $M$-ideals. In Banach algebras $M$-ideals are closed subalgebras but not necessarily ideals [Har, Theorem V.2.3]. In $\mathrm{C}^{*}$-algebras the sets of $M$-ideals and all closed two-sided ideals coincide.

If $I, J$ are $M$-ideals of $X$ then $I+J$ is an $M$-ideal [Har, Proposition 1.11]. This yields

Corollary 8.9 If a sublattice $Q$ of $\mathrm{Cl}(X)$ consists of $M$-deals then $\sqsubset=\prec=\subseteq$ in $Q$.

For a subset $S \subseteq \mathrm{Cl}(X), \operatorname{Alg} S$ is the algebra of all operators in $B(X)$ that leave all $L \in S$ invariant. For a subalgebra $\mathcal{A}$ of $B(X)$, Lat $\mathcal{A}$ is the lattice of all $\mathcal{A}$-invariant subspaces of $X$. A sublattice $Q$ of $\mathrm{Cl}(X)$ is reflexive if $Q=\operatorname{Lat}(\operatorname{Alg} Q)$ ). Similarly, a subalgebra $\mathcal{A}$ of $B(X)$ is reflexive if $\mathcal{A}=\operatorname{Alg}(\operatorname{Lat} \mathcal{A})$ ). $\operatorname{Alg} Q$ is a reflexive subalgebra of $B(X)$ and Lat $\mathcal{A}$ is a strongly closed, complete reflexive sublattice of $\mathrm{Cl}(X)$.

For $L \subseteq M$ in Lat $\mathcal{A}$, let $p: M \rightarrow M / L$ be the quotient map. Set $A_{p} p(x)=p(A x)$ for $A \in \mathcal{A}$ and $x \in M$. Then $\mathcal{A}_{p}=\left\{A_{p}: A \in \mathcal{A}\right\}$ is a subalgebra of the algebra $B(M / L)$ of all operators acting on $M / L$. To proceed further we need the following lemma.

Lemma 8.10 Let $L \subset M$ in Lat $\mathcal{A}$, let $p: M \rightarrow M / L$ and $K \in$ Lat $\mathcal{A}$.

(i) Suppose that $K \subseteq M$ and $L+K$ is closed. Set $q$ : $K \rightarrow K /(L \cap K)$. For $x \in K$, let $S q(x)=p(x)$. Then $p(K)$ is closed in $p(M), S$ is an invertible contraction from $q(K)$ onto $p(K)$ and

$$
S A_{q} q(x)=A_{p} S q(x) \text { for all } A \in \mathcal{A} \text { and } x \in K .
$$

(ii) Suppose that $L \subseteq K$ and $N:=M+K$ be closed. Set $q: N \rightarrow N / K$. For $x \in M$, let $T p(x)=q(x)$. Then $T$ is a contraction from $p(M)$ onto $q(N), \operatorname{ker} T=p(M \cap K)$ and

$$
T A_{p} p(x)=A_{q} T p(x) \text { for all } A \in \mathcal{A} \text { and } x \in M \text {. }
$$

Proof. (i) As $L+K$ is closed, $p(L+K)$ is closed. So $p(K)=p(L+K)$ is closed in $p(M)$. 
If $q(x)=q(y)$ then $x-y \in L \cap K$. So $p(x)=p(y)$. Thus $S$ is a well defined linear operator from $q(K)$ onto $p(K)$. If $S q(x)=0$ for $x \in K$, then $p(x)=0$. So $x \in L$. Thus $x \in L \cap K$ and $q(x)=0$. Hence $\operatorname{ker} S=\{0\}$. Moreover, $\|S\| \leq 1$, as

$$
\|p(x)\|_{M / L}=\inf _{t \in L}\|x+t\| \leq \inf _{t \in L \cap K}\|x+t\|=\|q(x)\|_{K /(L \cap K)} \text { for all } x \in K .
$$

Hence $S$ is an invertible contraction. For $A \in \mathcal{A}$ and $x \in K, A_{q} q(x)=q(A x)$, so that

$$
S A_{q} q(x)=S q(A x)=p(A x)=A_{p} p(x)=A_{p} S q(x) .
$$

(ii) As $N$ is closed, $q(M)=q(N)$ is a Banach space. If $p(x)=p(y)$ then $x-y \in L$. So $q(x)=q(y)$, as $L \subseteq K$. Thus $T$ is a well defined linear operator from $p(M)$ onto $q(N)$. If $T p(x)=q(x)=0$ then $x \in M \cap K$. So $\operatorname{ker} T=p(M \cap K)$. Moreover, $\|T\| \leq 1$, since

$$
\|q(x)\|_{N / K}=\inf _{t \in K}\|x+t\| \leq \inf _{t \in L}\|x+t\|=\|p(x)\|_{M / L} \text { for all } x \in M .
$$

We also have $T A_{p} p(x)=T p(A x)=q(A x)=A_{q} q(x)=A_{q} T p(x)$ for $A \in \mathcal{A}$ and $x \in M$.

Recall that a subalgebra $\mathcal{A}$ of $B(X)$ is irreducible on $L \in$ Lat $\mathcal{A}$, if $L$ has no non-trivial invariant subspaces. It is algebraically irreducible, if $L$ has no non-trivial invariant linear manifolds.

For a sublattice $Q$ of $\operatorname{Cl}(X)$, consider the following relations from $\operatorname{Ref}(Q)$ stronger than $<_{\mathfrak{g}}$ :

$$
\sqsubset_{\mathfrak{g}}=<_{\mathfrak{g}} \cap \sqsubset \text { and } \prec_{\mathfrak{g}}=<_{\mathfrak{g}} \cap \prec \text {, }
$$

that is, $L \sqsubset_{\mathfrak{g}} M$ if $L<_{\mathfrak{g}} M$ and $L \sqsubset M$; and $L \prec_{\mathfrak{g}} M$ if $L<_{\mathfrak{g}} M$ and $L \prec M$ for $L, M \in Q$.

However, for some pairs $(L, M), L<_{\mathfrak{g}} M$ implies $L \sqsubset_{\mathfrak{g}} M$, for some it implies $L \prec_{\mathfrak{g}} M$. For example, if $L<_{\mathfrak{g}} X$ then $L \sqsubset_{\mathfrak{g}} X$; if $\{0\}<_{\mathfrak{g}} M$ then $\{0\} \prec_{\mathfrak{g}} M$.

Theorem 8.11 $\sqsubset_{\mathfrak{g}}$ is an $\mathbf{H}$-relation and $\prec_{\mathfrak{g}}$ is a dual $\mathbf{H}$-relation in each reflexive sublattice.

Proof. If a sublattice $Q$ of $\operatorname{Cl}(X)$ is reflexive then $Q=$ Lat $\mathcal{A}$, where $\mathcal{A}=\operatorname{Alg} Q$.

Let $L \sqsubset_{\mathfrak{g}} M$ in $Q$ and $L \subseteq K \in Q$. As $L \sqsubset M$, we have that $N:=K+M=K \vee M$ is invariant and closed, i.e., $N \in Q$. If we show that $K \sqsubset_{\mathfrak{g}} N$ then, by Lemma 3.1(i), $\sqsubset_{\mathfrak{g}}$ is an H-relation.

By Proposition 8.8, $\sqsubset$ is an $\mathbf{H}$-relation. Hence it follows from Lemma 3.1(i) that $K \sqsubset N$. As $\sqsubset_{\mathfrak{g}}=<_{\mathfrak{g}} \cap \sqsubset$, we only need to prove $K<_{\mathfrak{g}} N$. As $L \subseteq M \cap K \subseteq M$ and $L<_{\mathfrak{g}} M$, either $L=M \cap K$ or $M \cap K=M$. In the second case $M \subseteq K$, so that $K=N$. Thus $K<_{\mathfrak{g}} N$ by (8.2).

Let now $L=M \cap K$ and $p: M \rightarrow M / L, q: N \rightarrow N / K$ be the quotient maps. The operator $T$ in Lemma 8.10(ii) is a contraction from $p(M)$ onto $q(N)$ and $\operatorname{ker} T=p(M \cap K)=p(L)=\{0\}$. Thus $T$ is invertible. Moreover, $T A_{p} p(x)=A_{q} T p(x)$ for all $A \in \mathcal{A}$ and $x \in M$.

As $L<_{\mathfrak{g}} M$, the algebra $\mathcal{A}_{p}$ on $M / L$ is irreducible. Hence, as $T$ is invertible, the algebra $\mathcal{A}_{q}$ on $N / K$ is irreducible, i.e., $K<_{\mathfrak{g}} N$. Thus $K \sqsubset_{\mathfrak{g}} N$, so that $\sqsubset_{\mathfrak{g}}$ is an H-relation.

Similarly, one can prove that $\prec_{\mathfrak{g}}$ is a dual $\mathbf{H}$-relation in $Q$.

Corollary 8.12 Let $Q=$ Lat $\mathcal{A}$ be a reflexive sublattice of $\mathrm{Cl}(X)$.

(i) $<_{\mathfrak{g}} \cap \ll_{n}$ is an $\mathbf{H H}$-relation in $Q$ for each $1 \leq n \leq \infty$.

(ii) Suppose that whenever $L<_{\mathfrak{g}} M$ in $Q$ then $\mathcal{A}_{p}$ is algebraically irreducible in $M / L$, where $p$ : $M \rightarrow M / L$. Then $<_{\mathfrak{g}}=\prec_{\mathfrak{g}}$ is a dual $\mathbf{H}$-relation. 
Proof. (i) By Lemma 8.4, the HH-relation $\ll_{n}$ is stronger than $\sqsubset$ and than $\prec$. By Theorem 8.11, $<_{\mathfrak{g}} \cap \sqsubset=\sqsubset_{\mathfrak{g}}$ is an $\mathbf{H}$-relation and $<_{\mathfrak{g}} \cap \prec=\prec_{\mathfrak{g}}$ is a dual H-relation. Hence, by Lemma 5.8, $<_{\mathfrak{g}} \cap \ll_{n}$ is an $\mathbf{H H}$-relation.

(ii) If $K \subset M$ in $Q$ then $L+K$ is an $\mathcal{A}$-invariant manifold and $L \subseteq L+K \subseteq M$. As $\mathcal{A}_{p}$ is algebraically irreducible in $M / L$, either $L+K=L$, or $L+K=M$. Hence $L+K$ is closed. Thus $L \prec M$. So $L \prec \mathfrak{g} M$.

Algebraically irreducible representations of Banach algebras were studied in the papers of Poguntke $[\mathrm{P}]$, Jeu and Tomiyama [JT], Radjavi $[\mathrm{R}]$, Barnes $[\mathrm{Ba}]$ and of many others.

\subsection{Superinvariant subspaces in Lat $\mathcal{A}$.}

A subspace $W \subseteq B(X)$ is a Lie subalgebra of $B(X)$ if the commutator $A B-B A \in W$ for all $A, B \in W$. Let $\mathcal{A}$ be a closed subalgebra of $B(X)$ and $Q=$ Lat $\mathcal{A}$. Then

$$
\text { Nor } \mathcal{A}=\{S \in B(X): S A-A S \in \mathcal{A} \text { for all } A \in \mathcal{A}\}
$$

is a closed Lie subalgebra of $B(X)$ and $\mathcal{A}^{\prime}+\mathcal{A} \subseteq$ Nor $\mathcal{A}$, where $\mathcal{A}^{\prime}$ is the commutant of $\mathcal{A}$ :

$$
\mathcal{A}^{\prime}=\{B \in B(X): A B-B A=0 \text { for all } A \in \mathcal{A}\} .
$$

A space in $Q$ is superinvariant ([K1]) if it is invariant for all operators in Nor $\mathcal{A}$.

For $S \in B(X)$, we define the map $\theta_{S}$ on $\mathrm{Cl}(X)$ by the formula $\theta_{S}(L)=e^{S} L$. If $S \in$ Nor $\mathcal{A}$ then (see [K2, Lemmas 2.1 and 2.3]) $\theta_{S}$ is an isomorphism of $Q$ and

$$
L \in Q \text { is superinvariant if and only if } \theta_{S}(L)=L \text { for all } S \in \text { Nor } \mathcal{A} \text {. }
$$

Recall (see (5.11) that $\theta_{S}$ preserves a relation $\ll$ in $Q$ if $\theta_{S}(L) \ll \theta_{S}(K) \Leftrightarrow L \ll K$ for $L, K \in Q$.

Proposition 8.13 Let all isomorphisms $\theta_{S}, S \in$ Nor $\mathcal{A}$, preserve a relation $\ll$ in $Q$. If $L \in Q$ is superinvariant then

(i) The subspaces $s^{\ll}(L)$ and $\sigma_{\ll}(L)$ in (8.3) and (8.4) are superinvariant. In particular, $s^{\ll}(\{0\})$ and $\sigma_{\ll}(X)$ are superinvariant.

(ii) If $\ll$ is an $\mathbf{H}$-relation then the $\ll^{\triangleright}$-radical in $[L, X]_{Q}$ is superinvariant. In particular, the $\ll^{\triangleright}$-radical in $Q$ is superinvariant.

(iii) If $\ll$ is a dual $\mathbf{H}$-relation then the dual $\ll^{\triangleleft}$-radical in $[\{0\}, L]_{Q}$ is superinvariant. In particular, the dual $\ll^{\triangleleft}$-radical in $Q$ is superinvariant.

Proof. (i) As $\theta_{S}$ preserve $\ll$ and as $\theta_{S}(L)=L$ for all $S \in$ Nor $\mathcal{A}$ by (8.13), Proposition 5.10 gives $\theta_{S}\left(s^{\ll}(L)\right)=s^{\ll}(L)$ and $\theta_{S}\left(\sigma_{\ll}(L)\right)=\sigma_{\ll}(L)$. Then, by $(8.13), s^{\ll}(L)$ and $\sigma_{\ll}(L)$ are superinvariant. Parts (ii) and (iii) have similar proofs and follow from Proposition 5.10.

Combining Theorem 8.1, Propositions 8.2 and 8.13 and (8.5), we obtain 
Corollary 8.14 Let $\ll$ be a relation in $Q=$ Lat $\mathcal{A}$ and let all $\theta_{S}, S \in$ Nor $\mathcal{A}$, preserve $\ll$.

(i) Suppose that $\mathcal{A}$ has no non-trivial superinvariant subspaces.

If $[\ll, X]_{Q} \neq\{X\}$ then $\sigma_{\ll}(X)=\{0\}$. If $[\{0\}, \ll]_{Q} \neq\{0\}$ then $s^{\ll}(\{0\})=X$.

(ii) Let $\ll$ be a dual $\mathbf{H}$-relation. If $\sigma_{\ll}(X)=\{0\}$ then the dual $\ll^{\triangleleft}$-radical $\mathfrak{p}=\{0\}$ and

1) for each $L \neq\{0\}$ in $Q$, there is $K \subset L$ in $Q$ such that $K \ll L$.

2) If $X$ is separable, there are $\ldots \ll Y_{n} \ll \ldots \ll Y_{1} \ll X$ in $Q$ such that $\cap_{n=1}^{\infty} Y_{n}=\{0\}$.

(iii) Let $\ll$ be an $\mathbf{H}$-relation. If $s^{\ll}(\{0\})=X$ then the $\ll^{\triangleright}$-radical $\mathfrak{r}=X$ and

1) for each $L \neq X$ in $Q$, there is $M \supset L$ in $Q$ such that $L \ll M$.

2) If $X$ is separable, there are $\{0\} \ll Y_{1} \ll \ldots \ll Y_{n} \ll \ldots$ in $Q$ such that $\overline{\sum_{n=1}^{\infty} Y_{n}}=X$.

Corollary 8.15 Isomorphisms $\theta_{S}, S \in$ Nor $\mathcal{A}$, preserve the relations $<_{\mathfrak{g}}, \sqsubset, \prec, \ll_{n}$ for $n \in \mathbb{N} \cup \infty$. Thus all results of Propositions 8.2 and 8.13 and Corollary 8.14 hold for these relations.

Proof. For $S \in$ Nor $\mathcal{A}$ and each $R \in Q$, set $R_{S}=e^{S} R$. Let $L<_{\mathfrak{g}} M$ in $Q$. If $L_{S} \varsubsetneqq K \varsubsetneqq M_{S}$ for some $K \in Q$, then $L \varsubsetneqq K_{-S} \varsubsetneqq M$ and $K_{-S} \in Q$, a contradiction. Thus $L_{S}<_{\mathfrak{g}} M_{S}$.

Let $L \sqsubset M$. Let $L_{S} \subset K \in Q$. Then $L \subset K_{-S}$, so that $M+K_{-S}$ is closed. Hence $\left(M+K_{-S}\right)_{S}=$ $M_{S}+K$ is closed. So $L_{S} \sqsubset M_{S}$. Conversely, if $L_{S} \sqsubset M_{S}$ then, by above, $L=\left(L_{S}\right)_{-S} \sqsubset\left(M_{S}\right)_{-S}=M$. So $\theta_{S}$ preserves the relation $\sqsubset$. Similarly, $\theta_{S}$ preserves the relation $\prec$.

If $L \subset M$ then $\operatorname{dim}(M / L)=\operatorname{dim}\left(M_{S} / L_{S}\right)$. So $\theta_{S}$ preserve all relations $\ll_{n}, n \in \mathbb{N} \cup \infty$.

Let us consider some cases where Corollary 8.14 can be applied.

Proposition 8.16 Let an operator $T \in B(X)$ have eigenvectors $\left\{e_{\lambda}\right\}_{\lambda \in \Lambda}$.

(i) If $X=\overline{\operatorname{span}\left(e_{\lambda}\right)_{\lambda \in \Lambda}}$ then the $\ll_{1}^{\triangleright}$-radical $\mathfrak{r}=s_{1}(\{0\})=X$ in Lat $T$ and all results of Corollary 8.14(iii) hold for the $\mathbf{H H}$-relation $\ll_{1}$ (see (8.8)).

(ii) Set $X_{\mu}=\overline{\operatorname{span}\left(e_{\lambda}\right)_{\mu \neq \lambda \in \Lambda}}$ for $\mu \in \Lambda$. If $\cap_{\mu \in \Lambda} X_{\mu}=\{0\}$ then the dual $\ll_{1}^{\triangleleft}$-radical $\mathfrak{p}=$ $\sigma_{\ll_{1}}(X)=\{0\}$ in Lat $T$ and all results of Corollary 8.14(ii) hold for the HH-relation $\ll_{1}$.

Proof. (i) As $\{0\} \ll_{1} \mathbb{C} e_{\lambda}$, we have $\mathbb{C} e_{\lambda} \in\left[\{0\}, \ll_{1}\right]$ (see (8.3)) for all $\lambda \in \Lambda$. By (8.4), $X=\vee \mathbb{C} e_{\lambda}=s^{\ll_{1}}(\{0\})$. By Corollary 8.15, $\mathfrak{r}=X$ and Corollary 8.14(iii) holds for $\ll_{1}$.

Part (ii) has a similar proof.

To illustrate Proposition 8.16, consider the following example.

Example 8.17 Let $X$ be a Hilbert space with basis $\left\{e_{n}\right\}_{n=1}^{\infty}$ and $T$ be the adjoint to the unilateral shift: $T e_{n}=e_{n-1}$. Let $\Lambda=\{\lambda \in \mathbb{C}:|\lambda|<1\}$. For each $\lambda \in \Lambda, e_{\lambda}=\left(1, \lambda, \lambda^{2}, \ldots\right) \in X$ is an eigenvector of $T: T e_{\lambda}=\lambda e_{\lambda}$, and $X=\overline{\operatorname{span}\left(e_{\lambda}\right)_{\lambda \in \Lambda}}$. Thus all results of Corollary 8.14(iii) hold.

Let the commutant $S^{\prime}$ of a set $S \subset B(X)$ contain projections $\left\{P_{\lambda}\right\}_{\lambda \in \Lambda}$, $\operatorname{dim} P_{\lambda}<\infty$. Set $Q=$ Lat $S$. All subspaces $X_{\lambda}=P_{\lambda} X$ belong to $\left[\{0\}, \ll_{\infty}\right]_{Q}$ and all $\left(\mathbf{1}-P_{\lambda}\right) X$ to $\left[\ll_{\infty}, X\right]_{Q}$. As in Proposition 8.16 , we obtain

Proposition 8.18 (i) If $\overline{\sum_{\lambda \in \Lambda} X_{\lambda}}=X$ then the $\ll_{\infty}^{\triangleright}$-radical $\mathfrak{r}=s^{\ll_{\infty}}(\{0\})=X$ in $Q$ and all results of Corollary 8.14(iii) hold for $\ll_{\infty}$.

(ii) If $\cap_{\lambda \in \Lambda}\left(\mathbf{1}-P_{\lambda}\right) X=\{0\}$ then the dual $\ll_{\infty}^{\triangleleft}$-radical $\mathfrak{p}=\sigma_{\ll_{\infty}}(X)=\{0\}$ in $Q$ and all results of Corollary 8.14(ii) hold for $\ll_{\infty}$. 
For example, suppose that the commutant $S^{\prime}$ contains a compact operator $T$. Then $\operatorname{Sp}(T)=$ $\left\{\lambda_{n}\right\}_{n=0}^{m}$ for $m \leq \infty$, where $\lambda_{0}=0$. The algebra $S^{\prime}$ contains projections $P_{n}, 1 \leq n \leq m$, such that $\operatorname{dim} P_{n}<\infty$. The subspaces $X_{n}=P_{n} X$ and $V_{n}=\left(1-P_{n}\right) X$ are $T$-invariant,

$$
X=X_{n}+V_{n}, \quad \operatorname{Sp}\left(\left.T\right|_{X_{n}}\right)=\left\{\lambda_{n}\right\} \text { and } \operatorname{Sp}\left(\left.T\right|_{V_{n}}\right)=\operatorname{Sp}(T) \backslash\left\{\lambda_{n}\right\} \text { for } 1 \leq n \leq m .
$$

Moreover, $X_{n} \subseteq V_{k}$ for $n \neq k$. So, for each $1 \leq k \leq m, X=Y_{k} \dot{+} \sum_{n=1}^{k} \dot{+} X_{n}$, where $Y_{k}=\cap_{n=1}^{k} V_{k}$, and all subspaces in the decompositions are $S$-invariant. Thus

1) if $\overline{\sum_{n \geq 1} X_{n}}=X$ then all results of Corollary 8.14(iii) hold for the relation $\ll_{\infty}$;

2) if $\cap_{n \geq 1} V_{n}=\{0\}$ then all results of Corollary 8.14(ii) hold for the relation $\ll_{\infty}$.

Proposition 8.19 The conditions $\overline{\sum_{n \geq 1} X_{n}}=X$ and $\cap_{n \geq 1} V_{n}=\{0\}$ above are not equivalent.

Proof. To show that $\overline{\sum_{n \geq 1} X_{n}}=X \not \cap_{n \geq 1} V_{n}=\{0\}$, consider the compact operator $T$ constructed in [Ha] (see also [N, p. 262]) on a Hilbert space $X$. It has the following properties:

$$
\operatorname{Sp}(T)=\left\{\lambda_{n}\right\}_{n=0}^{\infty}, \overline{\sum_{n \geq 1} X_{n}}=X, \text { where } X_{n}=\operatorname{Ker}\left(T-\lambda_{n} \mathbf{1}\right),
$$

and there exists a $T$-invariant subspace $E \neq\{0\}$ satisfying $\left.T\right|_{E} \neq 0$ and $\operatorname{Sp}\left(\left.T\right|_{E}\right)=0$. Let $P_{n}$ be the projections on $X_{n}$. Then (8.14) hold.

As the spectral radius $r\left(\left.T\right|_{E}\right)=0$, we have, for each $e \in E$,

$$
\left\|T^{k} e\right\|^{1 / k} \leq\left\|\left(\left.T\right|_{E}\right)^{k}\right\|^{1 / k} \longrightarrow r\left(\left.T\right|_{E}\right)=0, \text { as } k \longrightarrow \infty .
$$

Let $e \in E$ and $n \geq 1$. By (8.14), $e=x+y$, where $x \in X_{n}$ and $y \in V_{n}$. As $T$ and $P_{n}$ commute,

$$
\left\|T^{k} x\right\|^{1 / k}=\left\|T^{k} P_{n} e\right\|^{1 / k}=\left\|P_{n} T^{k} e\right\|^{1 / k} \leq\left\|P_{n}\right\|^{1 / k}\left\|T^{k} e\right\|^{1 / k} \stackrel{(8.15)}{\longrightarrow} 0,
$$

as $k \rightarrow \infty$. As $\operatorname{dim} X_{n}<\infty$ and $\operatorname{Sp}\left(\left.T\right|_{X_{n}}\right)=\lambda_{n} \neq 0$, it is easy to prove that $x=0$. Thus $e \in V_{n}$. Hence $\{0\} \neq E \subseteq \cap_{n \geq 1} V_{n}$. Thus $\overline{\sum_{n \geq 1} X_{n}}=X$ does not imply $\cap_{n \geq 1} V_{n}=\{0\}$.

To prove conversely that $\cap_{n \geq 1} V_{n}=\{0\} \not \overline{\sum_{n \geq 1} X_{n}}=X$, consider $A=T^{*}$. Then $\operatorname{Sp}(A)=$ $\left\{\lambda_{n}^{*}\right\}_{n=0}^{\infty}$. As in (8.14), let $Y_{n}$ and $U_{n}$ be $A$-invariant subspaces satisfying

$$
X=Y_{n} \dot{+} U_{n}, \operatorname{Sp}\left(\left.A\right|_{Y_{n}}\right)=\left\{\lambda_{n}^{*}\right\}, \operatorname{dim} Y_{n}<\infty \text { and } \operatorname{Sp}\left(\left.A\right|_{U_{n}}\right)=\operatorname{Sp}(A) \backslash\left\{\lambda_{n}^{*}\right\},
$$

for each $n$. Set $U=\cap_{n \geq 1} U_{n}$. Since $\operatorname{Sp}\left(\left.A\right|_{U}\right)=\{0\}$, we have as in (8.15) that $\left\|A^{k} u\right\|^{1 / k} \longrightarrow 0$, as $k \rightarrow \infty$, for each $u \in U$. Let $x \in X_{n}$ and $T x=\lambda_{n} x$ for $n \geq 1$. Then for all $u \in U$,

$$
\left|\lambda_{n}\right||(x, u)|^{1 / k}=\left|\left(T^{k} x, u\right)\right|^{1 / k}=\left|\left(x, A^{k} u\right)\right|^{1 / k} \leq\|x\|^{1 / k}\left\|A^{k} u\right\|^{1 / k} \longrightarrow 0,
$$

as $k \rightarrow \infty$. Hence $x \perp U$. If $T x=\lambda_{n} x+z$ and $T z=\lambda_{n} z$, then $z \perp U$ and, by (8.16), $x \perp U$. As $\operatorname{dim} X_{n}<\infty$, continuing this, we get that all $X_{n} \perp U$. As $\overline{\sum_{n \geq 1} X_{n}}=X$, we have $U=\{0\}$.

On the other hand, let $y \in Y_{n}$ be such that $A y=\lambda_{n}^{*} y$. Then, for each $e \in E$,

$$
\left|\lambda_{n}^{*}\right||(y, e)|^{1 / k}=\left|\left(A^{k} y, e\right)\right|^{1 / k}=\left|\left(y, T^{k} e\right)\right|^{1 / k} \leq\|y\|^{1 / k}\left\|T^{k} e\right\|^{1 / k} \stackrel{(8.15)}{\longrightarrow} 0,
$$

as $k \rightarrow \infty$. Hence $y \perp E$. If $A y=\lambda_{n}^{*} y+z$ and $A z=\lambda_{n}^{*} z$, then $z \perp U$ and, by (8.17), $y \perp U$. As $\operatorname{dim} Y_{n}<\infty$, continuing this, we get that all $Y_{n} \perp E$, so that $\overline{\sum_{n \geq 1} Y_{n}} \neq X$. Thus $\cap_{n \geq 1} U_{n}=\{0\}$ does not imply $\overline{\sum_{n \geq 1} Y_{n}}=X$. 


\subsection{Relation $<_{\mathfrak{g}}$ in sublattices of $\mathrm{Cl}(H)$, where $H$ is a Hilbert space}

In this section $X=H$ is a Hilbert space. First we consider reflexive sublattices of $\mathrm{Cl}(H)$, dim $H=\infty$, where $<_{\mathfrak{g}}$ is neither $\mathbf{H}$-, nor a dual $\mathbf{H}$-relation.

For $x, y \in H$, define the rank one operator $x \otimes y$ on $H$ by

$$
(x \otimes y) \xi=(\xi, x) y \text { for } \xi \in H .
$$

Let $F$ be a closed symmetric operator on $H$ with domain $D(F)$ and $F^{*}$ be its adjoint. Let $F^{*} \neq F$. Then $D(F) \varsubsetneqq D\left(F^{*}\right)$. For $u, v \in H, y \in D(F)$ and $x \in D\left(F^{*}\right)$,

$$
(x \otimes y)(u \otimes v)=(v, x)(u \otimes y) \text { and } F(x \otimes y)=x \otimes F y,(x \otimes y) F=F^{*} x \otimes y .
$$

Set $X=H \oplus H$ and let $\Omega=\{\{0\}, H \oplus\{0\},\{0\} \oplus H, X\}$. Consider

$$
\mathcal{A}=\operatorname{span}\left\{A(x, y)=\left(\begin{array}{cc}
x \otimes F y & 0 \\
0 & F x \otimes y
\end{array}\right) \text { for } x, y \in D(F)\right\} .
$$

By $(8.19), \mathcal{A}$ is a subalgebra of $B(X)$. For each closed operator $S$ on $H$ with domain $D(S)$,

$$
M_{S}=\{\widehat{\xi}=S \xi \oplus \xi: \xi \in D(S)\} \text { is a closed subspace of } X \text {. }
$$

Lemma 8.20 Let $\overline{F D(F)}=H$. Then Lat $\mathcal{A}=\Omega \cup\left(\cup_{t \in \mathbb{C} \backslash\{0\}} \mathcal{K}_{t}\right)$ and, for each $t$,

$$
\mathcal{K}_{t}=\left\{M_{t T}: F \subseteq T \subseteq F^{*}, T \text { is closed }\right\}=\left\{L: L \text { is a space, } M_{t F} \subseteq L \subseteq M_{t F^{*}}\right\} .
$$

Proof. Let $L \in$ Lat $\mathcal{A}$ and $L \notin \Omega$. As $F D(F)$ is dense in $H$, the subspaces $H \oplus\{0\}$ and $\{0\} \oplus H$ have no non-trivial $\mathcal{A}$-invariant subspaces. Hence $L=M_{S}$ for some closed operator $S \neq 0$ on $H$. As $A(x, y) \widehat{\xi} \subseteq M_{S}$ for all $x, y \in D(F)$ and $\xi \in D(S)$,

$$
(F x \otimes y) \xi \stackrel{(8.18)}{=} \sum_{i=1}^{n}(\xi, F x) y \in D(S) \text { and }(x \otimes F y) S=S(F x \otimes y) \stackrel{(8.19)}{=} F x \otimes S y .
$$

As $F D(F)$ is dense in $H$, we have $D(F) \subseteq D(S)$. As $(x \otimes F y) S=F x \otimes S y$, we have

$$
(x \otimes F y) S \eta \stackrel{(8.18)}{=}(S \eta, x) F y=(F x \otimes S y) \eta \stackrel{(8.18)}{=}(\eta, F x) S y \text { for } \eta \in D(S) .
$$

Fix $\eta$ and choose $x$ such that $(\eta, F x) \neq 0$. Then $S y=t F y$ for $y \in D(F)$ with $t=(S \eta, x) /(\eta, F x) \in$ C. As $0 \neq S$ is closed and $D(F) \subseteq D(S)$, we have $t \neq 0$. Thus $t F \subseteq S$.

Choose now $y \in D(F)$ in (8.20) such that $F y \neq 0$. Then $(S \eta, x)=t(\eta, F x)$ for all $x \in D(F)$ and $\eta \in D(S)$. Hence $\eta \in D\left(F^{*}\right)$ and $S \eta=t F^{*} \eta$. Thus $S \subseteq t F^{*}$ and $t F \subseteq S \subseteq t F^{*}$. Set $T=S / t$. Then $M_{S}=M_{t T}$ and $F \subseteq T \subseteq F^{*}$.

Conversely, let $F \subseteq T \subseteq F^{*}$. Then, for $A(x, y) \in \mathcal{A}, \xi \in D(T)$ and $t \in \mathbb{C} \backslash\{0\}$, we have

$$
A(x, y)(t T \xi \oplus \xi) \stackrel{(8.18)}{=} t(T \xi, x) F y \oplus(\xi, F x) y=(T \xi, x)(t T y \oplus y) \in M_{t T} .
$$

Thus $M_{t T} \in$ Lat $\mathcal{A}$ for all $t \in \mathbb{C} \backslash\{0\}$.

If $L$ is a subspace of $X$ and $M_{t F} \subseteq L \subseteq M_{t F^{*}}$, then there is a linear operator $T$ such that $D(F) \subseteq D(T) \subseteq D\left(F^{*}\right)$ and $L=M_{t T}$. As $L$ is closed, $T$ is closed. Thus $L \in \mathcal{K}_{t}$. 
Corollary 8.21 The sublattice Lat $\mathcal{A}$ of $\mathrm{Cl}(H)$ in Lemma 8.20 is not modular and the relation $<_{\mathfrak{g}}$ in Lat $\mathcal{A}$ is neither an $\mathbf{H}-$, nor a dual $\mathbf{H}$-relation.

Proof. As $\{0\}, H \oplus\{0\}, X, M_{F^{*}}, M_{F}$ form a pentagon in Lat $\mathcal{A}$ (see (6.3)), Lat $\mathcal{A}$ is not modular.

By Lemma 8.20, $\{0\}<_{\mathfrak{g}} H \oplus\{0\}<_{\mathfrak{g}} X$. If $<_{\mathfrak{g}}$ is an H-relation then, by Lemma 3.1(iii), $M_{F}=$ $\{0\} \vee M_{F}<_{\mathfrak{g}}(H \oplus\{0\}) \vee M_{F}=\overline{(H \oplus\{0\})+M_{F}}=X$. However, $M_{F} \nless_{\mathfrak{g}} X$, since $M_{F} \varsubsetneqq M_{F^{*}} \varsubsetneqq X$. Thus $<_{\mathfrak{g}}$ is not an H-relation.

If $<_{\mathfrak{g}}$ is a dual $\mathbf{H}$-relation then $\{0\}=(H \oplus\{0\}) \cap M_{F^{*}}<_{\mathfrak{g}} X \cap M_{F^{*}}=M_{F^{*}}$ by Lemma 3.1(ii). However, $\{0\} \nless_{\mathfrak{g}} M_{F^{*}}$, since $\{0\} \varsubsetneqq M_{F} \varsubsetneqq M_{F^{*}}$. Thus $<_{\mathfrak{g}}$ is not a dual H-relation.

As sublattices of a modular lattice are modular, it follows from Corollary 8.21 that $\mathrm{Cl}(H)$ is not modular if $\operatorname{dim} H=\infty$. On the other hand, $\mathrm{Cl}(H)=\operatorname{Ln}(H)$ if $\operatorname{dim} H<\infty$, so that $\mathrm{Cl}(H)$ is modular by Proposition 8.3. This yields (cf. Proposition 1.5.5 [K]).

Corollary 8.22 $\mathrm{Cl}(H)$ is a modular lattice if and only if $\operatorname{dim}(H)<\infty$.

We will now consider some sublattices of $\mathrm{Cl}(H)$, where $<_{\mathfrak{g}}$ is an $\mathbf{H H}$-relation. Denote by $P(H)$ the set of all orthogonal projections in $B(H)$. For $L \in \mathrm{Cl}(H), p_{L}$ denotes the projection on $L$. So $\mathrm{Cl}(H)$ can be identified with $P(H)$ and each sublattice of $\mathrm{Cl}(H)$ with a sublattice of $P(H)$.

A complete sublattice $Q$ of $\mathrm{Cl}(H)$ is a commutative subspace lattice (CSL) if all projections $p_{L}$, $L \in Q$, commute. For $p \in P(H)$, let $p^{\perp}=\mathbf{1}-p$. If $Q$ is CSL and $p, q \in Q$ then

$$
p \wedge q=p q, p \vee q=p+q-p q=p+p^{\perp} q .
$$

Theorem 8.23 Let $Q$ be a CSL in $\mathrm{Cl}(H)$. Then

(i) $Q$ has properties (JID) and (MID) (see (6.5)), so that it is modular.

(ii) $L+K \in Q$ for all $L, K \in Q$ and $<_{\mathfrak{g}}$ is an $\mathbf{H H}$-relation in $Q$.

(iii) $\sqsubset=\prec=\subseteq$ and $\sqsubset_{\mathfrak{g}}=\prec_{\mathfrak{g}}=<_{\mathfrak{g}}$ in $Q$.

(iv) $<_{\mathfrak{g}}^{\triangleleft}$ is an $\mathbf{H}$-order and a dual $\mathbf{R}$-order; $<_{\mathfrak{g}}$ is an $\mathbf{R}$-order and a dual $\mathbf{H}$-order.

(v) $\overline{<_{\mathfrak{g}}}=\widetilde{<_{\mathfrak{g}}}$ (see $(7.15)$ and $\left.(7.16)\right)$ is an $\mathbf{R R}$-order.

(vi) $Q$ is a union of disjoint intervals that have no gaps.

Proof. (i) It was noted in [Da, p. 357] that $Q$ has properties (JID) and (MID). For completeness, we prove this here directly. Let $G \subseteq Q$ and $z \in Q$. Set $q=\vee G$ and $p=\vee\{z x: x \in G\}$.

As $Q$ is a complete sublattice of $\mathrm{Cl}(H), q \in Q$. So

$$
p=\vee\{z x: x \in G\} \stackrel{(8.21)}{=} \vee\{z \wedge x: x \in G\} \stackrel{(6.4)}{\leq} z \wedge(\vee G)=z \wedge q \stackrel{(8.21)}{=} z q .
$$

We have $x=z x \oplus z^{\perp} x \leq p \oplus z^{\perp} q$ for all $x \in G$. Hence $q=\vee\{x: x \in G\} \leq p \oplus z^{\perp} q \stackrel{(8.22)}{\leq} z q \oplus z^{\perp} q=q$. So $z q=p$, i.e., $z \wedge(\vee G)=\vee\{z \wedge x: x \in G\}$. Thus $Q$ has property (MID) (see (6.5)).

If $G=\{x, y\}$ then $z \wedge(x \vee y)=(z \wedge x) \vee(z \wedge y)$. Thus $Q$ is distributive. So it is modular.

Set now $r=\wedge G$ and $s=\wedge\left\{z^{\perp} x: x \in G\right\}$. As $Q$ is complete, $r \in Q$. So

$$
\begin{aligned}
z \oplus z^{\perp} r \stackrel{(8.21)}{=} z \vee r & =z \vee(\wedge G) \stackrel{(6.4)}{\leq} \wedge\{z \vee x: x \in G\} \stackrel{(8.21)}{=} \wedge\left\{z \oplus z^{\perp} x: x \in G\right\} \\
& =z \oplus \wedge\left\{z^{\perp} x: x \in G\right\}=z \oplus s .
\end{aligned}
$$


So $z^{\perp} r \leq s$. For $x \in G$, we have $x=z x \oplus z^{\perp} x \geq z r \oplus s$. Therefore $r=\wedge\{x: x \in G\} \geq z r \oplus s$. So $z^{\perp} r \geq s$. Hence $z^{\perp} r=s$, so that $z \oplus z^{\perp} r=z \oplus s$. Thus

$$
z \vee(\wedge G) \stackrel{(8.21)}{=} z \oplus z^{\perp} r=z \oplus s \stackrel{(8.23)}{=} \wedge\{z \vee x: x \in G\}
$$

Therefore (see (6.5)) $Q$ has properties (JID).

(ii) Let $a=p_{L}, b=p_{K}$ for $L, K \in Q$. Then $L+K=L \oplus a^{\perp} K=L \oplus a^{\perp} b H$. As $Q$ is CSL, $a$ and $b$ commute. So $a^{\perp} b$ is a projection. Thus $a^{\perp} b H$ is a closed subspace of $K^{\perp}$. So $L+K$ is closed.

As $Q$ is modular, it follows from Corollary 6.7 that $<_{\mathfrak{g}}$ is an HH-relation in $Q$.

(iii) As $L+K \in Q$ for all $L, K \in Q$, it follows from (8.9) that $\sqsubset=\prec=\subseteq$. Hence, since $<_{\mathfrak{g}}=$ $<_{\mathfrak{g}} \cap \subseteq, \sqsubset_{\mathfrak{g}}=<_{\mathfrak{g}} \cap \sqsubset$ and $\prec_{\mathfrak{g}}=<_{\mathfrak{g}} \cap \prec$, we have $<_{\mathfrak{g}}=\sqsubset_{\mathfrak{g}}=\prec_{\mathfrak{g}}$.

As $Q$ is modular, has properties (JID) and (MID) (see (6.5)) and $<_{\mathfrak{g}}$ is an $\mathbf{H H}$-relation in $Q$, (iv) and (v) follow from Lemma 6.9 and Corollary 7.21. Part (vi) follows from Theorem 6.10.

The lattice of all finite projections in a $\mathrm{W}^{*}$-algebra is modular (Theorem V.1.37 [T]). So Corollary 6.7 yields

Corollary $\mathbf{8 . 2 4}<_{\mathfrak{g}}$ is an $\mathbf{H H}$-relation in the lattice of all finite projections in any $W^{*}$-algebra.

The lattice $\mathrm{Cl}(H) \approx P(H)$ has a large variety of $\mathbf{H}$ - and dual $\mathbf{H}$-relations which is difficult to describe. However, it is possible to describe all HH-relations in $P(H)$. To do this, recall that projections $p, q \in P(H)$ are equivalent $(p \sim q)$ if

$$
p=v v^{*} \text { and } q=v^{*} v \text { for some partial isometry } v .
$$

To introduce a new class of $\mathbf{H H}$-relations in $P(H)$, we need the following result.

Proposition 8.25 [K3] Let $p \leq q$ in $P(H)$. Then, for each projection $r \in P(H)$,

$$
\begin{aligned}
& (q-p)^{\perp} \leq(q \vee r-p \vee r)^{\perp} ; \\
& (q-p)^{\perp} \sim \mathfrak{z} \text { for some } \mathfrak{z} \in P(H), \text { and } \mathfrak{z} \leq(q \wedge r-p \wedge r)^{\perp} .
\end{aligned}
$$

Consider now the reflexive relations $\ll_{n}^{\perp}$ in $P(H)$, for $0 \leq n \leq \infty$, defined by the condition

$$
p \ll_{n}^{\perp} q \text { if } p \leq q \text { in } P(H) \text { and } \operatorname{dim}(q-p)^{\perp} \geq n \text {, so that } \ll_{0}^{\perp}=\leq .
$$

Theorem 8.26 The relations $\ll_{n}^{\perp}, 0 \leq n \leq \infty$, are $\mathbf{H H}$-relations in $P(H)$.

Proof. Let $p, q, r \in Q$ and $p \ll_{n}^{\perp} q$ for some $n$. Then $p \leq q$ and $\operatorname{dim}(q-p)^{\perp} \geq n$.

We have that $p \vee r, q \vee r$ belong to $Q$ and $p \vee r \leq q \vee r$. By $(8.25),(q-p)^{\perp} \leq(q \vee r-p \vee r)^{\perp}$. So $\operatorname{dim}(q \vee r-p \vee r)^{\perp} \geq n$. Hence $p \vee r \ll_{n}^{\perp} q \vee r$ by (8.27).

We have $p \wedge r, q \wedge r \in Q$ and $p \wedge r \leq q \wedge r$. By $(8.26),(q-p)^{\perp} \sim \mathfrak{z}$ and $\mathfrak{z} \leq(q \wedge r-p \wedge r)^{\perp}$ for some projection $\mathfrak{z}$ in $P(H)$. It follows from (8.24) that $\operatorname{dim}(q-p)^{\perp}=\operatorname{dim} \mathfrak{z}$. Hence $\operatorname{dim}(q \wedge r-p \wedge r)^{\perp} \geq n$. So $p \wedge r \ll_{n}^{\perp} q \wedge r$ by (8.27). Thus, by Definition $3.2 \ll_{n}^{\perp}$ is an HH-relation.

By Corollary 8.6, all $\ll_{n}, 1 \leq n \leq \infty$, are HH-relations in $\mathrm{Cl}(H) \approx P(H)$. The following result obtained [K3] in describes all $\mathbf{H H}$-relation in $P(H)$.

Theorem 8.27 For a separable $H$, each $\mathbf{H H}$-relation in $P(H)$ is either $\ll_{n}$, or $\ll_{n}^{\perp}$ for $n \in \mathbb{N} \cup \infty$. 


\section{$9 \quad$ H-relations in the lattices of ideals of Banach algebras}

\subsection{H-relations in the lattices of ideals of Banach algebras}

Let $A$ be a Banach algebra. Denote by $\operatorname{Id}_{A}$ the set of all closed two-sided ideals in $A$ (we call them ideals). Let $L R(A)$ be the Banach algebra of operators on $A$ generated by all operators of left and right multiplication by elements from $A$. Then $\operatorname{Id}_{A}=$ Lat $L R(A)$ is a sublattice of $\operatorname{Cl}(A)$.

The following Proposition was obtained in [Ru1, Example 4.6] and rediscovered in [Ki, Lemma 4.9(iv)] (see also [W, Lemma 8.1] and [Di, Proposition 2.4]).

Proposition 9.1 Let $L$ and $R$ be closed left and right ideals of $A$. If $L$ has a bounded right approximate identity (a. $i$.), or $R$ has a bounded left $a$. i., then $L+R$ is closed.

Corollary 9.2 Let a be a Banach algebra. If each $I \in \operatorname{Id}_{A}$ has a bounded left or right $a$. $i$., then $\prec=\sqsubset=\subseteq$ and $<_{\mathfrak{g}}=\prec_{\mathfrak{g}}=\sqsubset_{\mathfrak{g}}$ are $\mathbf{H H}$-relations in $\operatorname{Id}_{A}$.

Proof. It follows from (8.9) and Proposition 9.1 that $\prec=\sqsubset=\subseteq$. From this and from (8.2) and (8.11) we have $<_{\mathfrak{g}}=\prec_{\mathfrak{g}}=\sqsubset_{\mathfrak{g}}$ in $\operatorname{Id}_{A}$. By Theorem 8.11, it is an HH-relation.

Let $\ll$ be a relation in $\operatorname{Id}_{A}$. As in (8.3), for $L \in \operatorname{Id}_{A}$, let

$$
\sigma_{\ll}(L)=\cap\left\{J \in \operatorname{Id}_{A}: J \ll L\right\} \text { and } s^{\ll}(L)=\overline{\sum\left\{J \in \operatorname{Id}_{A}: L \ll J\right\}} .
$$

For an $\mathbf{H}$-relations $\ll, \ll^{\triangleright}$ is an $\mathbf{R}$-order in $\operatorname{Id}_{A}$ and the ideal

$$
\mathfrak{r}_{L} \stackrel{(2.7)}{=} \overline{\operatorname{span}\left\{J \in \operatorname{Id}_{A}: L \ll \triangleright J\right\}} \text { is the unique } \ll^{\triangleright} \text {-radical in }[L, A] \subseteq \operatorname{Id}_{A} .
$$

If $\ll$ is a dual $\mathbf{H}$-relations then $\ll^{\triangleleft}$ is a dual $\mathbf{R}$-order in $\operatorname{Id}_{A}$ and the ideal

$$
\mathfrak{p}_{L} \stackrel{(2.6)}{=} \cap\left\{J \in \operatorname{Id}_{A}: J \ll^{\triangleleft} L\right\} \text { is the unique dual } \ll^{\triangleleft} \text {-radical in }[\{0\}, L] \subseteq \operatorname{Id}_{A} .
$$

Corollary 9.3 The results of Theorem 8.1 and Proposition 8.2 hold for $X=A$, where $Q=\operatorname{Id}_{A}$ is a sublattice of $\mathrm{Cl}(A)$ and the word "spaces" replaced by "ideals" of $A$.

By Corollary 8.6, all $\ll_{n}$ are $\mathbf{H H}$-relations in $\operatorname{Id}_{A}$ and $\ll_{\infty}$ is an $\mathbf{H H}$-order. Proposition 8.2 yields

Corollary 9.4 Let $A$ be a separable Banach algebra.

(i) Let $\sigma_{\ll_{\infty}}(A)=\{0\}$. Then there is a chain ... $I_{n} \subset \ldots \subset I_{0}=A$ of ideals of finite codimension in $A$ such that $\cap_{n} I_{n}=\{0\}$. Each $\{0\} \neq J \in \operatorname{Id}_{A}$ contains $I \in \operatorname{Id}_{A}$ such that $0<\operatorname{dim}(J / I)<\infty$.

(ii) Let $s^{\ll_{\infty}}(\{0\})=A$. Then there is a chain $\{0\}=I_{0} \subset . . \subset I_{n}$.. of ideals such that $\operatorname{dim} I_{n}<\infty$ and $\overline{\sum_{n=1}^{\infty} I_{n}}=A$. Each $A \neq J \in \operatorname{Id}_{A}$ is contained in $I \in \operatorname{Id}_{A}$ such that $0<\operatorname{dim}(I / J)<\infty$.

Since ideals of a Banach algebra $A$ are precisely invariant subspaces of the algebra $L R(A)$, it is natural to call superinvariant subspaces of $A$ by superideals. Thus $I \in \operatorname{Id}_{A}$ is a superideal of $A$, if (see (8.12)) it is invariant for all operators $S$ from the Lie subalgebra Nor $L R(A)$ of the algebra $B(A)$ :

$$
\text { Nor } L R(A)=\{S \in B(A): S T-T S \in L R(A) \text { for all } T \in L R(A)\} .
$$

A bounded operator $\delta \in B(A)$ is a derivation on $A$ if $\delta(a b)=\delta(a) b+a \delta(b)$ for $a, b \in A$. 
Theorem 9.5 (i) Nor $L R(A)$ contains all derivations of $A$.

(ii) If $I \in \operatorname{Id}_{A}$ then each operator $S \in \operatorname{Nor} L R(A)$ maps $\overline{I^{2}}$ in $I: S x \in I$ for $x \in \overline{I^{2}}$.

(iii) If $\overline{I^{2}}=I$ (in particular, if $I$ has a left (right) a. i.) then $I$ is a superideal.

Proof. (i) Let $L_{a}, R_{a}$ be the operators of left and right multiplication by $a \in A$ on $A$. Then

$$
\left[\delta, L_{a}\right] x=\delta L_{a} x-L_{a} \delta(x)=\delta(a x)-a \delta(x)=\delta(a) x=L_{\delta(a)} x \text { for all } x \in A .
$$

Hence $\left[\delta, L_{a}\right]=L_{\delta(a)} \in L R(A)$. Similarly, $\left[\delta, R_{a}\right]=\delta R_{a}-R_{a} \delta=R_{\delta(a)} \in L R(A)$. Since the algebra $L R(A)$ is generated by the sums of products of the operators $L_{a}$ and $R_{b}$ and since $[\delta, T S]=$ $[\delta, T] S+T[\delta, S]$ for all $T, S \in B(A)$, we have $\delta \in \operatorname{Nor} L R(A)$.

(ii) Let $S \in$ Nor $L R(A)$ and $a, b \in I$. Then

$$
S(a b)=S R_{b} a=R_{b} S a+\left[S, R_{b}\right] a=(S a) b+\left[S, R_{b}\right] a .
$$

As $S a \in A$ and $b \in I$, we have $(S a) b \in I$. As $\left[S, R_{b}\right] \in L R(A)$ by $(9.2)$, we have $\left[S, R_{b}\right] a \in I$. Hence $S(a b) \in I$. Since $S$ is linear and bounded, it maps $\overline{I^{2}}$ into $I$. Part (iii) follows from (ii).

To see an example of a Banach algebra that has no non-trivial superideal, consider a Banach space $A$ with trivial multiplication: $a b=0$ for $a, b \in A$. Then $A$ is a Banach algebra, $L R(A)=\{0\}$ and Nor $L R(A)=B(A)$. As $B(A)$ has no non-trivial invariant subspaces, $A$ has no superideals. On the other hand, all closed subspaces of $A$ are ideals of $A$.

Denote by $\Sigma_{A}$ the set of all closed subalgebras of $A$ of finite codimension.

Proposition 9.6 For each Banach algebra $A, \sigma_{\ll_{\infty}}(A)=\cap_{S \in \Sigma_{A}} S$.

Proof. As $\left\{J \in \operatorname{Id}_{A}: J \ll_{\infty} A\right\} \subseteq \Sigma_{A}$, we have $\cap_{S \in \Sigma_{A}} S \subseteq \sigma_{\ll_{\infty}}(A)$ by (9.1). In [L] it was proved that each $S \in \Sigma_{A}$ contains an ideal of finite codimension. Thus $\sigma_{\ll_{\infty}}(A) \subseteq \cap_{S \in \Sigma_{A}} S$. So $\sigma_{\ll_{\infty}}(A)=\cap_{S \in \Sigma_{A}} S$.

\subsection{H-relations in the lattices of all ideals of $\mathrm{C}^{*}$-algebras.}

Let $A$ be a $C^{*}$-algebra. Since ideals of $A$ have bounded a.i., $I+J \in \operatorname{Id}_{A}$ for all $I, J \in \operatorname{Id}_{A}$, by Proposition 9.1 (see $[\mathrm{D}]$ ). Thus $\operatorname{Id}_{A}$ is a sublattice (not a complete sublattice) of $\operatorname{Ln}(A)$. So $\operatorname{Id}_{A}$ is a modular lattice and $<_{\mathfrak{g}}$ is an $\mathbf{H H}$-relation in $\operatorname{Id}_{A}$ by Proposition 8.3. It also follows from Corollary 9.2 that the relations $\prec$, $\sqsubset$ and $\subseteq$ coincide in $\operatorname{Id}_{A}$ and $<_{\mathfrak{g}}=\prec_{\mathfrak{g}}=\sqsubset_{\mathfrak{g}}$.

The complete lattice $\operatorname{Id}_{A}$ has many $\mathbf{H}$-relations (some of them stronger than $<_{\mathfrak{g}}$ ) that depend on the nature of the quotient ideals $J / I$. A large variety of such relations was investigated in [KST4]. In this section we briefly consider some of them.

Let $\mathfrak{A}$ be the set of all $\mathrm{C}^{*}$-algebras. We say that a subclass $P$ of $\mathfrak{A}$ is a property, if

$$
\{0\} \in P \text { and } A \in P \text { implies } B \in P \text { for all } B \approx A \text {. }
$$

If $A \in P$, we say that $A$ has property $P$, or $A$ is a $P$-algebra. For example, the classes $C C R$ of all CCR-algebras and $G C R$ of all GCR-algebras are properties.

A standard way to define a relation in $\operatorname{Id}_{A}$ is to consider a property $P$ and to write

$$
I \ll_{P} J \text { if } I \subseteq J \text { in } \operatorname{Id}_{A} \text { and } J / I \text { is a } P \text {-algebra, i.e., } J / I \in P \text {. }
$$

A property $P$ on $\mathfrak{A}$ is lower stable if $A \in P$ implies that all $I \in \operatorname{Id}_{A}$ belong to $P$; $P$ is upper stable if $A \in P$ implies that the quotients $A / I \in P$ for all $I \in \operatorname{Id}_{A}$. 
Theorem 9.7 (i) A property $P$ is upper stable if and only if $\ll_{P}$ is an $\mathbf{H}$-relation. Then $\ll_{P}^{\triangleright}$ is an $\mathbf{R}$-order and $\operatorname{Id}_{A}$ has the $\ll_{P}^{\triangleright}$-radical $\mathfrak{r}_{P}:\{0\} \ll_{P}^{\triangleright} \mathfrak{r}_{P} \overleftarrow{\ll}_{P}^{\triangleright} A$.

(ii) A property $P$ is lower stable if and only if $\ll_{P}$ is a dual $\mathbf{H}$-relation. Then $\ll_{P}^{\triangleleft}$ is a dual $\mathbf{R}$-order and $\operatorname{Id}_{A}$ has the dual $\ll_{P}^{\triangleleft}$-radical $\mathfrak{p}_{P}:\{0\} \overrightarrow{\ll_{P}^{\triangleleft}} \mathfrak{p}_{P} \ll_{P}^{\triangleleft} A$.

Proof. (i) Let $I \ll_{P} J$ in $\operatorname{Id}_{A}, I \neq J$, and $I \subseteq K \in \operatorname{Id}_{A}$. Then $(K \cap J) / I$ is an ideal of $J / I$. As $J / I \in P$ and $P$ is upper stable, the quotient $(J / I) /((K \cap J) / I) \in P$. As $(J / I) /((K \cap J) / I) \approx$ $J /(K \cap J)$, we have $J /(K \cap J) \in P$. By Corollary 1.8.4 [D], $J /(K \cap J) \approx(J+K) / K$, so that $(J+K) / K \in P$. Thus $K \ll_{P}(J+K)=J \vee K$. So, by Lemma 3.1, $\ll_{P}$ is an H-relation.

Conversely, if $\ll_{P}$ is an $\mathbf{H}$-relation and $A \in P$ then $\{0\} \ll_{P} A$ by (9.4). Hence, for $I \in \operatorname{Id}_{A}$, $I \ll_{P}(A+I)=A$ by Lemma 3.1. Thus $A / I \in P$ by (9.4). So $P$ is upper stable.

The rest of (i) follows from Theorem 5.5. Part (ii) can be proved similarly.

Now we can treat $\operatorname{Id}_{A}$ as a lattice with two relations: $\subseteq$ and $\ll_{P}$. With this approach we can construct a large variety of $\mathbf{H}$ - and dual $\mathbf{H}$-relations and, using methods of the lattice theory, obtain many results in the theory of $\mathrm{C}^{*}$-algebras. Thus Theorems 8.1 and 9.7 yield

Theorem 9.8 I. Let $P$ be an upper stable property, so that $\ll_{P}$ is an $\mathbf{H}$-relation. Let $A \in \mathfrak{A}$.

(i) The radical $\mathfrak{r}_{P}$ is the largest ideal in $A$ such that there is an ascending $\ll_{P}$-series of ideals from $\{0\}$ to $\mathfrak{r}_{P}$.

(ii) If $\mathfrak{r}_{P} \nsubseteq I \neq A$, then there is $J \in I d_{A}$ such that $J / I$ is a $P$-algebra.

(iii) $\mathfrak{r}_{P}$ is the smallest out of all ideals $J$ such that $A / J$ has no $P$-ideals.

II. Let $P$ be a lower stable property, so that $\ll_{P}$ is a dual $\mathbf{H}$-relation. Let $A \in \mathfrak{A}$.

(i) The dual radical $\mathfrak{p}_{P}$ is the smallest ideal in $A$ such that there is an descending $\ll_{P}$-series of ideals from $A$ to $\mathfrak{p}_{P}$.

(ii) If $\{0\} \neq I \nsubseteq \mathfrak{p}_{P}$ then there is $J \in I d_{A}$ such that $I / J$ is a P-algebra.

(iii) $\mathfrak{p}_{P}$ is the largest ideal such that all its quotients are not P-algebras.

Proof. I. Parts (i) and (ii) follow from Theorem 8.1 (ii).

(iii). By Theorem 8.1 (ii), $\mathfrak{r}_{P}$ has no $\ll_{P}$-successor. So $A / \mathfrak{r}_{P}$ has no $P$-ideals.

Let $A / J$ have no $P$-ideals for $J \in \operatorname{Id}_{A}$. If $\mathfrak{r}_{P} \nsubseteq J$ then, by Theorem 8.1 (ii) 2 ), $A / J$ has $P$-ideals. So $\mathfrak{r}_{P} \subseteq J$ which proves (iii). The proof of part II is similar.

Each automorphism $\phi$ of a $\mathrm{C}^{*}$-algebra $A$ generates a lattice automorphism $\widetilde{\phi}$ of $\operatorname{Id}_{A}$. For a property $P$, let $I \ll_{P} J$ in $\operatorname{Id}_{A}$, i.e., $J / I \in P$. Then $\widetilde{\phi}(I) \subseteq \widetilde{\phi}(J)$ and the map $\widehat{\phi}: J / I \rightarrow \widetilde{\phi}(J) / \widetilde{\phi}(I)$ defined by $\widehat{\phi}(x+I)=\phi(x)+\widetilde{\phi}(I)$ for $x \in J$, is an isomorphism, i.e., $J / I \approx \widetilde{\phi}(J) / \widetilde{\phi}(I)$, so that $\widetilde{\phi}(J) / \widetilde{\phi}(I) \in P$, i.e., $\widetilde{\phi}(I) \ll_{P} \widetilde{\phi}(J)$. As $\phi^{-1}$ is also an automorphism, $\widetilde{\phi}(I) \ll_{P} \widetilde{\phi}(J)$ implies $I \ll_{P} J$. Thus $\widetilde{\phi}$ preserves $\ll_{P}$. So Proposition 5.10 yields

Corollary 9.9 Let $\ll_{P}$ be an $\mathbf{H}$-relation with $\ll_{P}^{\triangleright}$-radical $\mathfrak{r}_{P}$ and $\ll_{S}$ be a dual $\mathbf{H}$-relation with dual $\ll_{S}^{\triangleleft}$-radical $\mathfrak{p}_{S}$. Then $\widetilde{\phi}\left(\mathfrak{r}_{P}\right)=\mathfrak{r}_{P}$ and $\widetilde{\phi}\left(\mathfrak{p}_{S}\right)=\mathfrak{p}_{S}$ for all automorphisms $\phi$ of $A$.

By Propositions 4.2.4 and 4.3.5 [D], the properties $C C R$ and $G C R$ of all CCR- and GCRalgebras are lower and upper stable. For the property $C C R$ this gives a well-known result that 
the radical $\mathfrak{r}_{C C R}$ is the largest GCR-ideal in $A$ and $A / \mathfrak{r}_{C C R}$ has no CCR-ideals. Moreover, if $\mathfrak{r}_{C C R} \nsubseteq I \neq A$ then there is $J \in \operatorname{Id}_{A}$ such that $J / I$ is a CCR-algebra. However, the property of all NGCR-algebras is lower, but not upper stable (see $4.7 .4 \mathrm{~b}$ ) and c) [D]).

Consider now some more lower and upper stable properties. A unital $A \in \mathfrak{A}$ has real rank zero $[\mathrm{BP}]$ if its invertible selfadjoint elements are dense in the set of all selfadjoint elements of $A$. A non-unital algebra is real rank zero if its unitization is real rank zero. Denote by $R Z$ the class of all real rank zero algebras. Then $R Z$ is a property.

Let $A \in R Z$. By Corollary $2.8[\mathrm{BP}]$, each hereditary $\mathrm{C}^{*}$-subalgebra of $A$ also belongs to $R Z$. As each ideal $I$ of $A$ is hereditary (Theorem I.5.3 [Da1]), $I \in R Z$. Let $p: A \rightarrow A / I$. As $\|p(x)\|_{A / I} \leq\|x\|_{A}$, it follows that $A / I$ also belongs to $R Z$. This yields

Corollary 9.10 The property $R Z$ is lower and upper stable.

Recall that $A \in \mathfrak{A}$ is approximately finite-dimensional (AF algebra) if it is the closure of an increasing union of finite-dimensional *-subalgebras. It is nuclear if, for each $\mathrm{C}^{*}$-algebra $B, A \odot B$ has only one $\mathrm{C}^{*}$-norm. Finite-dimensional and all commutative $\mathrm{C}^{*}$-algebras, $C(\mathcal{H})$, all $\mathrm{AF}$ and all $\mathrm{C}^{*}$-algebras of type I are nuclear. Then (see Section III.4 [Da1] and Corollary XV.3.4 [T])

Theorem 9.11 (i) $A C^{*}$-algebra $A$ is nuclear if and only if each ideal $I$ and $A / I$ are nuclear.

(ii) $A C^{*}$-algebra $A$ is an AF algebra if and only if each ideal $I$ and $A / I$ are AF algebras.

Denote by $A F$ and $N U$ the classes of all $\mathrm{AF}$ and of all nuclear $\mathrm{C}^{*}$-algebras. By Theorem 9.11, they are lower and upper stable properties. Hence Theorem 9.7 yields

Corollary 9.12 For each $A \in \mathfrak{A}, \ll_{C C R}, \ll_{G C R}, \ll_{R Z}, \ll_{A F}, \ll_{N U}$ are $\mathbf{H H}$-relations in $\operatorname{Id}_{A}$.

We will now discuss transitivity of the relation $\ll_{P}$ for a property $P$.

Lemma 9.13 The relation $\ll_{P}$ is transitive in all lattices $\operatorname{Id}_{A}, A \in \mathfrak{A}$, if and only if, for all $B \in \mathfrak{A}$ and all $S \in \operatorname{Id}_{B}$, the condition $S, B / S \in P$ implies $B \in P$.

Proof. Let $B \in \mathfrak{A}$ and $S \in \operatorname{Id}_{B}$. Let $S, B / S \in P$. Then $\{0\} \ll_{P} S \ll_{P} B$. If $\ll_{P}$ is transitive in all lattices $\operatorname{Id}_{A}, A \in \mathfrak{A}$, it is transitive in $\operatorname{Id}_{B}$. So $\{0\} \ll_{P} B$. Thus $B \in P$.

Conversely, let $A \in \mathfrak{A}$ and $K \ll_{P} I \ll_{P} J$ in $\operatorname{Id}_{A}$. Then $I / K, J / I \in P$ and $J / I \approx(J / K) /(I / K)$. Let, for all $B \in \mathfrak{A}$ and $S \in \operatorname{Id}_{B}, S, B / S \in P$ implies $B \in P$. Set $B=J / K$ and $S=I / K$. As $S, B / S \approx J / I \in P$, we have $J / K=B \in P$. So $K \ll_{P} J$. Thus $\ll_{P}$ is transitive in all $\operatorname{Id}_{A}, A \in \mathfrak{A}$.

The relation $\ll_{C C R}$ is not transitive. Indeed, let $B=C(H)+\mathbb{C} \mathbf{1}_{H}$. Then $C(H)$ and $B / C(H) \approx$ $\mathbb{C} 1$ are CCR-algebras, but $B$ is not a CCR-algebra. So, by Lemma $9.13, \ll_{C C R}$ is not transitive.

Similarly, $\ll_{R Z}$ is not transitive, since $A \in R Z$ if and only if all $I \in \operatorname{Id}_{A}$ and $A / I$ are $R Z$-algebras and all projections in $A / I$ lift to projections in $A$ (Theorem $3.14[\mathrm{BP}]$ ). On the other hand, $\ll_{A F}$ and $\ll_{N U}$ are transitive by Theorem 9.11. It is also easy to show that $\ll_{G C R}$ is transitive.

If an HH-relation $\ll_{P}$ is not transitive then, generally, the $\ll_{P}^{\triangleright}$-radical $\mathfrak{r}_{P}$ is not a $P$-algebra. For example, if $B=C(H)+\mathbb{C} \mathbf{1}_{H}$, we have $\{0\} \ll_{C C R} C(H) \ll_{C C R} B$ and $\mathfrak{r}_{C C R}=B$ is a GCR- and not a CCR-algebra. Even if $\ll_{P}$ is transitive, $\mathfrak{r}_{P}$ may still be not a $P$-algebra. It is a $P$-algebra if and only if the inductive limits of ascending series of $P$-ideals of $A$ are $P$-algebras ([KST4]). For example, AF and nuclear algebras have this property (see [W, p. 17]). 
Corollary 9.14 For $A \in \mathfrak{A}$, let $\mathfrak{r}_{A F}$ be the $\ll_{A F}^{\triangleright}$-radical and $\mathfrak{r}_{N U}$ be the $\ll_{N U}^{\triangleright}$-radical.

(i) ([KST4]) The relations $\ll_{A F}$, $\ll_{N U}$ are $\mathbf{R}$-orders in $\mathrm{Id}_{A}$ : $\ll_{A F}=\ll_{A F}^{\triangleright}$ and $\ll_{N U}=\ll_{N U}^{\triangleright}$.

(ii) ([ST], [KST4]) $\mathfrak{r}_{A F}$ is the largest AF-algebra and $\mathfrak{r}_{N U}$ is the largest nuclear algebra in $A$.

\subsection{Relation $\ll_{\infty}$ in the lattices of Lie ideals of Banach Lie algebras.}

A complex Lie algebra $A$ with Lie multiplication $[\cdot, \cdot]$ is a Banach Lie algebra, if it is a Banach space in some norm $\|\cdot\|$ and $\|[a, b]\| \leq C\|a\|\|b\|$ for some $C>0$ and for all $a, b \in A$.

A subspace $I$ of $A$ is a Lie subalgebra if $[a, b] \in I$ for all $a, b \in I$; it is a Lie ideal if $[a, b] \in I$ for all $a \in I, b \in A$. Banach algebras are Banach Lie algebras with Lie multiplication $[a, b]=a b-b a$.

The set $\mathrm{Li}_{A}$ of all closed Lie ideals of $A$ is a complete lattice of invariant subspaces of the subalgebra of $B(A)$ generated by all operators $\operatorname{ad}(a): x \rightarrow[a, x]$ on $A$. As in (8.8), the HH-relation $\ll_{\infty}$ in $\operatorname{Li}_{A}$ is defined by: $I \ll_{\infty} J$ if $\operatorname{dim}(J / I)<\infty$. Then Corollaries 9.3 and 9.4 hold in this setting with two-sided ideals replaced by Lie ideals.

A linear bounded operator $\delta$ on $A$ is a Lie derivation if

$$
\delta([a, b])=[\delta(a), b]+[a, \delta(b)] \text { for } a, b \in A .
$$

The set $\mathfrak{D}(A)$ of all Lie derivations on $A$ is a closed Lie subalgebra of the algebra $B(A)$ of all bounded operators on $A$. Each $a \in A$ defines a Lie derivation ad $(a)$ on $A$.

An ideal $I \in \mathrm{Li}_{A}$ is called characteristic if $\delta(I) \subseteq I$ for all $\delta \in \mathfrak{D}(A)$. By (9.5), the centre of $A$ is a characteristic Lie ideal. If $A$ is commutative then $\{0\}$ and $A$ are the only characteristic Lie ideals of $A$, as $\mathfrak{D}(A)=B(A)$ and only $\{0\}$ and $A$ are invariant for $B(A)$.

If $\delta \in \mathfrak{D}(A)$ then $e^{t \delta}=\sum_{n=0}^{\infty} t^{n} \delta^{n} / n$ ! for $t \in \mathbb{R}$, is a one-parameter group of bounded Lie automorphism of $A: e^{t \delta}([a, b])=\left[e^{t \delta}(a), e^{t \delta}(b)\right]$ for $a, b \in A$. Hence

$$
I \in \operatorname{Li}_{A} \Rightarrow e^{t \delta}(I) \in \operatorname{Li}_{A} \text { for all } t \in \mathbb{R} \text { and } \delta \in \mathfrak{D}(A)
$$

Moreover, since $\delta(a)=\lim _{t \rightarrow 0}\left(e^{t \delta}(a)-a\right) / t \in I$ for all $a \in I$, it follows that

$I$ is a characteristic Lie ideal if and only if $e^{t \delta}(I)=I$ for all $t \in \mathbb{R}$ and $\delta \in \mathfrak{D}(A)$.

Denote by $\mathrm{Li}_{A}^{\text {ch }}$ the subset of $\mathrm{Li}_{A}$ of all closed characteristic Lie ideals of $A$. We have that

$$
\text { if } J \in \mathrm{Li}_{A}^{\text {ch }} \text { and } I \text { is a characteristic Lie ideal of } J \text {, then } I \in \mathrm{Li}_{A}^{\text {ch }} \text {. }
$$

Indeed, $\left.\delta\right|_{J} \in \mathfrak{D}(J)$ for all $\delta \in \mathfrak{D}(A)$, as $J \in \mathrm{Li}_{A}^{\text {ch }}$. Then $\delta(I)=\left.\delta\right|_{J}(I) \subseteq I$, as $I \in \mathrm{Li}_{J}^{\mathrm{ch}}$. Thus, as $\operatorname{ad}(a) \in \mathfrak{D}(A)$, for each $a \in A,[a, I]=\operatorname{ad}(a)(I) \subseteq I$. Hence $I \in \mathrm{Li}_{A}^{\text {ch }}$.

The existence of Lie and characteristic Lie ideals of finite codimension in Banach Lie algebras was studied in [KST2]. We will use below the following result obtained there.

Theorem 9.15 If a Banach non-commutative Lie algebra has a closed proper Lie subalgebra of finite codimension, then it has a proper closed characteristic Lie ideal of finite codimension.

As the intersection and the closed sum of any family of characteristic Lie ideals is a characteristic Lie ideal, $\mathrm{Li}_{A}^{\text {ch }}$ is a complete sublattice of $\mathrm{Li}_{A}$. Denote by $\mathfrak{p}_{\infty}^{\text {ch }}(A)$ the dual $\ll_{\infty}^{\triangleleft}$-radical in $\mathrm{Li}_{A}^{\text {ch }}$ and by $\Sigma_{A}$ the set of all closed Lie subalgebras of finite codimension in $A$.

We now prove the following version of Proposition 9.6 for Banach Lie algebras. 
Theorem 9.16 Let $\mathrm{Li}_{A}^{\mathrm{ch}}$ have no commutative infinite-dimensional Lie ideals and $\cap_{L \in \Sigma_{A}} L=\{0\}$. Then, for each $\{0\} \neq K \in \mathrm{Li}_{A}^{\mathrm{ch}}$, there is $I \in \mathrm{Li}_{A}^{\text {ch }}$ such that $K \neq I \ll_{\infty} I$, and $\mathfrak{p}_{\infty}^{\mathrm{ch}}(A)=\{0\}$.

Proof. Set $\mathfrak{p}=\mathfrak{p}_{\infty}^{\text {ch }}(A)$. Let $\{0\} \neq K \in \operatorname{Li}_{A}^{\text {ch }}$. If $\operatorname{dim} K<\infty$ then $\{0\} \ll_{\infty} K$ and $\{0\} \in \operatorname{Li}_{A}^{\text {ch }}$.

Let $\operatorname{dim} K=\infty$. As $\cap_{L \in \Sigma_{A}} L=\{0\}$, there is $L \in \Sigma_{A}$ that does not contain $K$. Then $K \cap L$ is a proper closed Lie subalgebra of $A$. Replacing $M$ by $A$ in Lemma 8.4(i), we have that $K \cap L$ has finite codimension in $K$. By our assumption, $K$ is non-commutative. So, by Theorem 9.15 , there is $I \in \mathrm{Li}_{K}^{\text {ch }}$ of finite codimension, i.e., $\operatorname{dim}(K / I)<\infty$. By $(9.8), I \in \mathrm{Li}_{A}^{\text {ch }}$, so that $I \ll_{\infty} K$ in $\mathrm{Li}_{A}^{\text {ch }}$.

If $\mathfrak{p} \neq\{0\}$ then, by above, $I \ll_{\infty} \mathfrak{p}$ for some $\mathfrak{p} \neq I \in \mathrm{Li}_{A}^{\text {ch }}$ which contradicts Theorem 8.1(i).

The condition that $\mathrm{Li}_{A}^{\text {ch }}$ has no commutative infinite-dimensional Lie ideals in Theorem 9.16 is essential. To show this, consider the following construction of Banach Lie algebras [Bo, Sec 1.8].

Let $X$ be a Banach space and $\mathcal{L}$ a closed Lie subalgebra of $B(X)$. The direct sum $A=\mathfrak{L} \oplus^{\text {id }} X$ (semidirect product of $\mathfrak{L}$ and $X$ ) endowed with Lie multiplication and norm

$$
[(a ; x),(b ; y)]=([a, b] ; a y-b x) \text { and }\|(a ; x)\|=\max \{\|a\|,\|x\|\} \text { for } a, b \in \mathfrak{L}, x, y \in X,
$$

is a Banach Lie algebra. Clearly, $J=\{0\} \oplus^{\text {id }} X$ is a closed commutative Lie ideal of $A$.

Example 9.17 Let $\operatorname{dim} X=\infty, \operatorname{dim} \mathfrak{L}<\infty$ and let $\mathfrak{L}$ have no invariant subspaces in $X$. Then $\cap_{L \in \Sigma_{A}} L=\{0\}$. However, $\mathfrak{p}_{\infty}^{\mathrm{ch}}(A)=J \neq\{0\}$, as $\mathrm{Li}_{A}^{\mathrm{ch}}$ has a commutative Lie ideal $J$.

Proof. If $Y$ is a closed subspace of codimension 1 in $X$ then $\{0\} \oplus^{\text {id }} Y \in \Sigma_{A}$, as $\operatorname{dim} \mathfrak{L}<\infty$. Since the intersection of all such Lie subalgebras is $\{0\}$, we have $\cap_{L \in \Sigma_{A}} L=\{0\}$.

As $\mathfrak{L}$ is irreducible in $X$, each $K \in \mathrm{Li}_{A}$ has form $K=I \oplus^{\text {id }} X$, where $I \in \mathrm{Li}_{\mathfrak{L}}$; and $J$ is the unique minimal non-zero closed Lie ideal of $A$. By $(9.6), e^{t \delta}(J)$ is a minimal Lie ideal for each $t \in \mathbb{R}$ and $\delta \in \mathfrak{D}(A)$, so that $e^{t \delta}(J)=J$. By $(9.7), J \in \mathrm{Li}_{A}^{\text {ch }}$. Thus the condition in Theorem 9.16 fails and the theorem does not hold since $\{0\} \neq \mathfrak{p}_{\infty}^{\mathrm{ch}}(A)=J \ll_{\infty} A$.

Let $\mathfrak{p}_{\infty}(\mathfrak{L})$ be the dual $\ll_{\infty}^{\triangleleft}$-radicals in $\operatorname{Li}_{\mathfrak{L}}$ and $\mathfrak{p}_{\infty}(X)$ be the dual $\ll_{\infty}^{\triangleleft}$-radicals in Lat $\mathfrak{L}$ - the lattice of all $\mathfrak{L}$-invariant subspaces in $X$. Let $\mathfrak{p}_{\infty}(A)$ be the dual $\ll_{\infty}^{\triangleleft}$-radical in $\mathrm{Li}_{A}$.

Proposition 9.18 Let $A=\mathfrak{L} \oplus^{\text {id }} X$. If $\mathfrak{p}_{\infty}(\mathfrak{L})=\{0\}$ then $\mathfrak{p}_{\infty}(A)=\{0\} \oplus^{\text {id }} \mathfrak{p}_{\infty}(X)$.

Proof. As $\mathfrak{p}_{\infty}(\mathfrak{L})=\{0\}$, there is a descending $\ll_{\infty}$-series of Lie ideals $\left(I_{\lambda}\right)_{1 \leq \lambda \leq \gamma}$ of $\mathfrak{L}$ from $\mathfrak{L}$ to $\{0\}$. Then $C_{\mathfrak{L}}=\left\{I_{\lambda} \oplus^{\text {id }} X\right\}_{1 \leq \lambda \leq \gamma}$ is a descending $\ll_{\infty}$-series of Lie ideals of $A$ from $A$ to $\{0\} \oplus^{\text {id }} X$.

Let $C_{X}=\left\{Y_{\omega}\right\}_{1 \leq \omega \leq \alpha}$ be a descending $\ll_{\infty}$-series of subspaces in Lat $\mathfrak{L}$ from $X$ to $\mathfrak{p}_{\infty}(X)$. As $\mathfrak{p}_{\infty}(X)$ has no invariant subspaces of finite codimension, the Lie ideal $J=\{0\} \oplus^{\text {id }} \mathfrak{p}_{\infty}(X)$ contains no Lie ideals of $A$ of finite codimension and $C=C_{\mathfrak{L}} \cup C_{X}$ is a descending $\ll_{\infty}$-series of Lie ideals from $A$ to $J$. So, by Corollary $9.3, \mathfrak{p}_{\infty}(A)=J$.

\section{References}

[A-I] S. A. Amitsur, A general theory of radicals. I. Radicals in complete lattices, Amer. J. Math. 74 (1952), 774-786.

[A-II] S. A. Amitsur, A general theory of radicals. II. Radicals in rings and bicategories, Amer. J. Math. 76 (1954), 100-125. 
[A-III] S. A. Amitsur, A general theory of radicals. III. Applications, Amer. J. Math. 76 (1954), 126136.

[Ba] B. A. Barnes, When is a representation of a Banach *-algebra Naimark-related to a $*_{-}$ representation?, Pacific J. Mathematics, 72(1977), No 1, 5-25.

[Bo] N. Bourbaki, "Groupes et algebres de Lie", Hermann, Paris VI, 1971.

[BP] L.G. Brown and G.K. Pedersen, C*-Algebras of Real Rank Zero, J. Funct. Anal., 99 (1991), 131-149.

[Da] K.R. Davidson, "Nest algebras", Pitman Research Notes in Mathematics Series, Longman, 1988.

[Da1] K.R. Davidson, "C*-algebras by Example", AMS, Providence, Rhode Island, 1991.

[DS] N. Dunford and J.T. Schwartz, "Linear operators", Interscience Publishers, New York, London, 1958.

[D] J. Dixmier, "Les C*-algebres et leurs representations", Paris, Gauthier-Villars Editeur, 1969.

[Di1] P.G. Dixon, Topologically irreducible representations and radicals in Banach algebras, Proc. London Math. Soc. (3), 74 (1997), 174-200.

[Di] P. G. Dixon, Non-closed sums of closed ideals in Banach algebras, Proc. AMS, 128, 12 (2000), 3647-3654.

[G] G. Grätzer, "Lattice Theory: Foundation", Birkhäuser, 2011.

[Gr] M. Gray, "A radical approach to algebra", Addison-Wesley, London, Ontario, 1970.

[Ha] H. L. Hamburger, Uber die Zerlegung des Hilbertschen Raumes durch vollstetige lineare Transformationen, Math. Nachr., 1951, 4, 56-69.

[Har] P. Harmand, D. Werner, W. Werner,." M-ideals in Banach spaces and Banach algebras", Springer-Verlag, 1992.

[H] I. N. Herstein, "Noncommutative rings", The Carus Math. Monog., 15, Math. Ass. of Am., 1971.

[JT] M. de Jeu and J. Tomiyama, Algebraically irreducible representations and structure space of the Banach algebra associated with a topological dynamical system, arXiv:1407.8329v1[math.OA] 31 Jul 2014.

[K] G. Kalambach, "Orthomodular Lattices", Academic Press, London, New York, 1983.

[Ka] I. Kaplansky, The structure of certain operator algebras, Trans. AMS, 70 (1951), 219-255.

[Ke] J. L. Kelley, "General topology", Van Nostrand Co., Inc., Princeton, N.J., 1955.

[Ki] E. Kirchberg, On restricted perturbations in inverse images and a description of normalizer algebras in $\mathrm{C}^{*}$-algebras, J. Funct. Anal., 129 (1995), 1-34.

[K1] E. Kissin, Invariant subspaces for derivations, Proc. of Amer. Math. Soc., 102(1988), 95-101.

[K2] E. Kissin, On lattices of invariant subspaces of operator algebras, J. Oper. Theory, 18(1987), 373-392.

[K3] E. Kissin, Relations and trails in lattices of projections in $\mathrm{W}^{*}$-algebras, Proc. Edinburgh Math. Soc., 2019, to appear.

[KST2] E. Kissin, V.S. Shulman and Yu. V. Turovskii, Banach Lie algebras with Lie subalgebras of finite codimension have Lie ideals, J. London Math. Soc., 80 (2009), 603-626.

[KST3] E. Kissin, V.S. Shulman and Yu. V. Turovskii, Topological radicals and Frattini theory of Banach Lie algebras, Integr. Equ. Oper. Theory, 74 (2012), 51-121.

[KST4] E. Kissin, V.S. Shulman and Yu. V. Turovskii, Relations and radicals in lattices of ideals of $\mathrm{C}^{*}$-algebras, to appear.

[KST5] E. Kissin, V.S. Shulman and Yu. V. Turovskii, On theory of topological radicals, Contemporary Mathematics, Fundamental directions, 64, No 3 (2018), 490-546. 
[L] T. Laffey, On the structure of algebraic algebras, Pacific J. Math., 62(1976), 461-471.

$[\mathrm{N}] \quad$ N. K. Nikol'skii, Invariant subspaces in the theory of operators and the theory of functions, Itogi Nauki i Techniki, Matematicheskii Analiz, v. 12, 1974, 199-412. [In Russian]

[P] D. Poguntke, Algebraically irreducible representations of $L^{1}$-algebras of exponential Lie groups, Duke Math. J., 50(1983), No 4, 1077-1106.

[R] H. Radjavi, Nonselfadjoint representations of $\mathrm{C}^{*}$-algebras, Proc. of AMS, 47(1975), 133136.

[Ro] S. Roman, "Lattices and ordered sets", Springer, 2008.

[Ru1] W. Rudin, Spaces of type $H^{\infty}+C$, Ann. Inst. Fourier, Grenoble, 25 (1975), 99-125. (MR 57:13692; errata MR 57:7134)

[Sa] S. Sakai, "C*-algebras and $W^{*}$-algebras", Springer-Verlag, New-York Heidelberg, 1998.

[Sch] H. H. Schaefer, "Topological Vector Spaces", Springer, New-York Heidelberg Berlin, 1971.

[ST] V.S. Shulman and Yu. V. Turovskii, Topological radicals, V. From algebra to spectral theory, Operator Theory Advances and Applications 233, Algebraic Methods in Functional analysis (2014), 171-280.

[Sk] L. A. Skornjakov, "Elements of Lattice Theory", Hindustan Publ. Corp., Delhi, 1977.

[T] M. Takesaki, "Theory of operator algebra", Springer, Berlin Heidelberg New York, 2001.

[W] S. Wassermann, Exact $\mathrm{C}^{*}$-algebras and related topics, Res. Inst. Mathem., Global Analysis Res. Center, Seoul National Univ., Lecture Note Series, 19, 1994.

E. Kissin: STORM, London Metropolitan University, 166-220 Holloway Road, London N7 8DB, Great Britain; e-mail: e.kissin@londonmet.ac.uk

V. S. Shulman: Department of Mathematics, Vologda State University, Vologda, Russia; e-mail: shulman.victor80@gmail.com

Yu. V. Turovskii: Konakovo, Russia, e-mail: yuri.turovskii@gmail.com 